\title{
Spectral stability of inviscid columnar vortices
}

\author{
Thierry Gallay \\ Institut Fourier \\ Université Grenoble Alpes, CNRS \\ 100 rue des Maths \\ 38610 Gières, France \\ Thierry.Gallay@univ-grenoble-alpes.fr
}

\author{
Didier Smets \\ Laboratoire Jacques-Louis Lions \\ Sorbonne Université \\ 4, place Jussieu \\ 75005 Paris, France \\ Didier.Smets@sorbonne-universite.fr
}

June 6, 2019

Dedicated to the memory of Louis N. Howard

\begin{abstract}
Columnar vortices are stationary solutions of the three-dimensional Euler equations with axial symmetry, where the velocity field only depends on the distance to the axis and has no component in the axial direction. Stability of such flows was first investigated by Lord Kelvin in 1880, but despite a long history the only analytical results available so far provide necessary conditions for instability under either planar or axisymmetric perturbations. The purpose of this paper is to show that columnar vortices are spectrally stable with respect to three-dimensional perturbations with no particular symmetry. Our result applies to a large family of velocity profiles, including the most common models in atmospheric flows and engineering applications. The proof is based on a homotopy argument, which allows us to concentrate in the spectral analysis of the linearized operator to a small neighborhood of the imaginary axis, where unstable eigenvalues can be excluded using integral identities and a careful study of the so-called critical layers.
\end{abstract}

\section{Introduction}

An interesting open question in hydrodynamic stability theory is whether the balance between the centrifugal force and the pressure gradient in axisymmetric vortex flows may lead to an instability even if the vorticity profile is monotone and the velocity field has no axial component. For incompressible perfect fluids, partial answers have been obtained under additional symmetry assumptions. For instance, in the restricted framework of two-dimensional flows, radially symmetric vortices are known to be stable if the vorticity distribution is a monotone function of the distance to the vortex center [20, 19], but even in that idealized situation no sharp stability criterion seems to be available. In the three-dimensional case, the simplest vortex-like equilibria are columnar vortices, namely axisymmetric flows with no vertical velocity and no dependence upon the vertical coordinate. In such flows, all streamlines are horizontal circles centered on the vertical symmetry axis. According to a celebrated result of Rayleigh [21, columnar vortices are stable with respect to axisymmetric perturbations if the square of the velocity circulation along the streamlines is a nondecreasing function of the distance to the symmetry axis, and that condition is actually sharp [24].

A natural question arises from these centennial results: When the vorticity profile is monotone and Rayleigh's condition is satisfied, are columnar vortices stable against three-dimensional 
perturbations with no particular symmetry? Although instabilities have never been observed experimentally or numerically for such vortices in the absence of axial flow, we could not find in the literature even a plausible formal argument supporting the affirmative answer, see Section 1.3 below for a short historical discussion. In the present paper, we give a rigorous proof of spectral stability for a large family of inviscid columnar vortices without imposing any symmetry assumption on the class of allowed perturbations. We thus provide an answer to an important question that dates back to the pioneering work of Lord Kelvin [27], who was the first to investigate the three-dimensional stability of vortex columns.

Before stating our results, we first describe the precise framework. We start from the incompressible Euler equation in the whole space $\mathbb{R}^{3}$ :

$$
\partial_{t} u+(u \cdot \nabla) u=-\nabla p, \quad \operatorname{div} u=0,
$$

where $u=u(x, t) \in \mathbb{R}^{3}$ denotes the fluid velocity and $p=p(x, t) \in \mathbb{R}$ the internal pressure. We mainly consider the vorticity $\omega(x, t)=\operatorname{curl} u(x, t)$, which describes the local rotation of the fluid particles. Since we are interested in the stability of axially symmetric flows, it is convenient to use cylindrical coordinates $(r, \theta, z)$ defined by $x_{1}=r \cos \theta, x_{2}=r \sin \theta$, and $x_{3}=z$. The velocity and vorticity fields are then decomposed as follows:

$$
\begin{aligned}
& u=u_{r}(r, \theta, z, t) e_{r}+u_{\theta}(r, \theta, z, t) e_{\theta}+u_{z}(r, \theta, z, t) e_{z}, \\
& \omega=\omega_{r}(r, \theta, z, t) e_{r}+\omega_{\theta}(r, \theta, z, t) e_{\theta}+\omega_{z}(r, \theta, z, t) e_{z},
\end{aligned}
$$

where $e_{r}, e_{\theta}, e_{z}$ are unit vectors in the radial, azimuthal, and vertical directions, respectively. In these coordinates, the vorticity equation $\partial_{t} \omega+(u \cdot \nabla) \omega-(\omega \cdot \nabla) u=0$ becomes

$$
\begin{aligned}
& \partial_{t} \omega_{r}+(u \cdot \nabla) \omega_{r}-(\omega \cdot \nabla) u_{r}=0, \\
& \partial_{t} \omega_{\theta}+(u \cdot \nabla) \omega_{\theta}-(\omega \cdot \nabla) u_{\theta}=\frac{1}{r}\left(u_{r} \omega_{\theta}-u_{\theta} \omega_{r}\right), \\
& \partial_{t} \omega_{z}+(u \cdot \nabla) \omega_{z}-(\omega \cdot \nabla) u_{z}=0,
\end{aligned}
$$

where $u \cdot \nabla=u_{r} \partial_{r}+\frac{1}{r} u_{\theta} \partial_{\theta}+u_{z} \partial_{z}$ and $\omega \cdot \nabla=\omega_{r} \partial_{r}+\frac{1}{r} \omega_{\theta} \partial_{\theta}+\omega_{z} \partial_{z}$. The velocity field satisfies the incompressibility condition

$$
\frac{1}{r} \partial_{r}\left(r u_{r}\right)+\frac{1}{r} \partial_{\theta} u_{\theta}+\partial_{z} u_{z}=0,
$$

and can be expressed in terms of the vorticity by solving the linear elliptic system

$$
\frac{1}{r} \partial_{\theta} u_{z}-\partial_{z} u_{\theta}=\omega_{r}, \quad \partial_{z} u_{r}-\partial_{r} u_{z}=\omega_{\theta}, \quad \frac{1}{r} \partial_{r}\left(r u_{\theta}\right)-\frac{1}{r} \partial_{\theta} u_{r}=\omega_{z} .
$$

\subsection{Columnar vortices}

Columnar vortices are stationary solutions of (1.1)-(1.4) of the particular form

$$
u=V(r) e_{\theta}, \quad \omega=W(r) e_{z}, \quad p=P(r),
$$

where $V$ is the velocity profile and $W$ the vorticity distribution. The pressure $P$ inside the vortex is determined, up to an irrelevant additive constant, by the centrifugal balance $r P^{\prime}(r)=V(r)^{2}$. Instead of $V$, we prefer using the angular velocity $\Omega(r)=V(r) / r$, which has the same physical dimension as the vorticity $W$. As a consequence of (1.4), we have

$$
W(r)=\frac{1}{r} \partial_{r}(r V(r))=r \Omega^{\prime}(r)+2 \Omega(r) .
$$


Here are typical examples that are often considered in the literature:

1. The Rankine vortex:

$$
\Omega(r)=\left\{\begin{array}{ll}
1 & \text { if } \quad r \leq 1, \\
r^{-2} & \text { if } \quad r \geq 1,
\end{array} \quad W(r)=\left\{\begin{array}{lll}
2 & \text { if } & r<1 \\
0 & \text { if } & r>1
\end{array}\right.\right.
$$

As is clear from (1.7), the flow of Rankine's vortex corresponds to a rigid rotation for $r<1$ and an irrotational motion for $r>1$. Although non-physical because of the singularity at $r=1$, this flow is relatively easy to analyze mathematically due to the very simple form of the vorticity distribution $W$, which is a piecewise constant function. The dynamical stability of Rankine's vortex was first investigated by L. Kelvin as early as 1880 [27].

2. The Kaufmann-Scully vortex:

$$
\Omega(r)=\frac{1}{1+r^{2}}, \quad W(r)=\frac{2}{\left(1+r^{2}\right)^{2}}, \quad r>0 .
$$

This smooth vortex is characterized by a relatively slow decay of the vorticity distribution as $r \rightarrow \infty$. It has also a very simple analytical form, and is often used as a model for vortices that appear in atmospheric flows or in laboratory experiments, see e.g. [2, Section 3.3.4].

3. The Lamb-Oseen vortex:

$$
\Omega(r)=\frac{1}{r^{2}}\left(1-e^{-r^{2}}\right), \quad W(r)=2 e^{-r^{2}}, \quad r>0 .
$$

Among all solutions of the form (1.5), the Lamb-Oseen vortex plays a distinguished role in connection with the long-time asymptotics of viscous planar flows. Indeed, if viscosity is taken into account, it is known that all localized distributions of vorticity evolve toward a Gaussian vorticity profile as $t \rightarrow+\infty$, see [10]. In particular, the Lamb-Oseen vortex is the only one in the above family which corresponds to a self-similar solution of the Navier-Stokes equations.

Remark 1.1. Throughout this paper, it is understood that all independent and dependent variables in the Euler equations (1.1) are dimensionless. Examples (1.7)-(1.9) are normalized so that the vortex core has a diameter of size $\mathcal{O}(1)$, but that choice can be modified by a simple rescaling. Also, we assume without loss of generality that all vortices are normalized so that $\Omega(0)=1$, which implies $W(0)=2$.

To study the dynamical stability of the columnar vortex (1.5), we look for solutions of (1.2), (1.3) of the form

$$
u(r, \theta, z, t)=r \Omega(r) e_{\theta}+\tilde{u}(r, \theta, z, t), \quad \omega(r, \theta, z, t)=W(r) e_{z}+\tilde{\omega}(r, \theta, z, t),
$$

where $\Omega=V / r$ is the angular velocity of the vortex and $W$ the vorticity distribution given by (1.6). Inserting this Ansatz into (1.2), neglecting the quadratic terms in $\tilde{u}$ and $\tilde{\omega}$, and finally dropping the tildes for notational simplicity, we arrive at the linearized evolution equations

$$
\begin{aligned}
\partial_{t} \omega_{r}+\Omega(r) \partial_{\theta} \omega_{r} & =W(r) \partial_{z} u_{r}, \\
\partial_{t} \omega_{\theta}+\Omega(r) \partial_{\theta} \omega_{\theta} & =W(r) \partial_{z} u_{\theta}+r \Omega^{\prime}(r) \omega_{r}, \\
\partial_{t} \omega_{z}+\Omega(r) \partial_{\theta} \omega_{z} & =W(r) \partial_{z} u_{z}-W^{\prime}(r) u_{r},
\end{aligned}
$$

which are the starting point of our analysis. Of course, the linear relations (1.3), (1.4) still hold for the perturbed velocity and vorticity. 
It is a classical observation that equations (1.10) can be considered as a self-contained evolution system for the vorticity $\omega$, provided the velocity $u$ is expressed in terms of $\omega$ by solving the linear elliptic system (1.3), (1.4). Once this is done, we can rewrite (1.10) in the compact form

$$
\partial_{t} \omega=L \omega,
$$

where $L$ is a vector-valued, nonlocal, first order differential operator. Our purpose is to study the spectral properties of that operator, and to show that $L$ has no spectrum outside the imaginary axis under general assumptions on the angular velocity $\Omega$ or the vorticity distribution $W$.

Another fundamental remark is that system (1.2)-(1.4) is invariant under rotations about the vertical axis, and under translations along that axis. Using a Fourier series expansion with respect to the angular variable $\theta$ and a Fourier transform in the vertical variable $z$, we are led to consider velocities and vorticities of the following particular form

$$
u(r, \theta, z, t)=u_{m, k}(r, t) e^{i m \theta} e^{i k z}, \quad \omega(r, \theta, z, t)=\omega_{m, k}(r, t) e^{i m \theta} e^{i k z},
$$

where $m \in \mathbb{Z}$ is the angular Fourier mode and $k \in \mathbb{R}$ is the vertical wave number. Here $u, \omega$ are complex-valued functions, but we impose that $\overline{u_{m, k}}=u_{-m,-k}$ and $\overline{\omega_{m, k}}=\omega_{-m,-k}$ so as to obtain real functions after summing over all possible values of $m, k$. Dropping the subscripts $m, k$ for notational simplicity, we see that the perturbation equations (1.10) translate into

$$
\begin{aligned}
& \left(\partial_{t}+i m \Omega(r)\right) \omega_{r}=W(r) i k u_{r}, \\
& \left(\partial_{t}+i m \Omega(r)\right) \omega_{\theta}=W(r) i k u_{\theta}+r \Omega^{\prime}(r) \omega_{r}, \\
& \left(\partial_{t}+i m \Omega(r)\right) \omega_{z}=W(r) i k u_{z}-W^{\prime}(r) u_{r} .
\end{aligned}
$$

In addition, the following relations hold :

$$
\begin{array}{ll}
\omega_{r} & =\frac{i m}{r} u_{z}-i k u_{\theta}, \\
\omega_{\theta} & =i k u_{r}-\partial_{r} u_{z}, \\
\omega_{z} & =\frac{1}{r} \partial_{r}\left(r u_{\theta}\right)-\frac{i m}{r} u_{r},
\end{array} \quad \text { and } \quad \frac{1}{r} \partial_{r}\left(r u_{r}\right)+\frac{i m}{r} u_{\theta}+i k u_{z}=0 .
$$

As before, we can rewrite (1.13) in the compact form

$$
\partial_{t} \omega=L_{m, k} \omega,
$$

assuming that the velocity $u=u_{m, k}$ in (1.13) is expressed in terms of the vorticity $\omega=\omega_{m, k}$ by solving the linear relations (1.14) with appropriate boundary conditions. The main properties of the Biot-Savart map $\omega_{m, k} \mapsto u_{m, k}$ obtained in this way will be recalled in Section 6.1, Being an integral operator acting on functions of the sole variable $r$, the generator $L_{m, k}$ in (1.15) is of course easier to study than the original three-dimensional differential operator $L$ in (1.11).

\subsection{Statement of the results}

To state our results in a precise way, we first specify our hypotheses on the unperturbed columnar vortex. We find it convenient to formulate these assumptions at the level of the vorticity profile $W$. Note that, in view of (1.6), the angular velocity $\Omega$ can be expressed in terms of $W$ by the formula

$$
\Omega(r)=\frac{1}{r^{2}} \int_{0}^{r} W(s) s \mathrm{~d} s, \quad r>0,
$$

and the derivative of $\Omega$ is in turn given by

$$
\Omega^{\prime}(r)=\frac{W(r)-2 \Omega(r)}{r}=\frac{1}{r^{3}} \int_{0}^{r} W^{\prime}(s) s^{2} \mathrm{~d} s, \quad r>0 .
$$


In what follows, we denote $\mathbb{R}_{+}=(0, \infty)$ and $\overline{\mathbb{R}}_{+}=[0, \infty)$.

Assumption H1: The vorticity profile $W: \overline{\mathbb{R}}_{+} \rightarrow \mathbb{R}_{+}$is a $\mathcal{C}^{1}$ function satisfying $W^{\prime}(0)=0$, $W^{\prime}(r)<0$ for all $r>0$, and the total circulation

$$
2 \pi \Gamma=2 \pi \int_{0}^{\infty} W(r) r \mathrm{~d} r
$$

of the columnar vortex is finite.

Under assumption H1 the angular velocity profile $\Omega \in \mathcal{C}^{1}\left(\overline{\mathbb{R}}_{+}\right) \cap \mathcal{C}^{2}\left(\mathbb{R}_{+}\right)$given by (1.16) is positive and satisfies $\Omega(0)=W(0) / 2, \Omega^{\prime}(0)=0, \Omega^{\prime}(r)<0$ for all $r>0$, and $\Omega(r) \sim \Gamma / r^{2}$ as $r \rightarrow \infty$. In particular, the Rayleigh function $\Phi:[0, \infty) \rightarrow \mathbb{R}$ defined by

$$
\Phi(r)=2 \Omega(r) W(r), \quad r \geq 0,
$$

is positive everywhere. As a matter of fact, in our framework assumption $\mathrm{H} 1$ corresponds exactly to the combination of Rayleigh's condition [21] and of the two-dimensional stability criterion [20, 19]. We supplement it with the following:

Assumption H2: The $\mathcal{C}^{1}$ function $J: \mathbb{R}_{+} \rightarrow \mathbb{R}_{+}$defined by

$$
J(r)=\frac{\Phi(r)}{\Omega^{\prime}(r)^{2}}, \quad r>0,
$$

satisfies $J^{\prime}(r)<0$ for all $r>0$ and $r J^{\prime}(r) \rightarrow 0$ as $r \rightarrow \infty$.

This second assumption is more technical in nature, and certainly more difficult to justify. We first observe that it is satisfied for the Kaufmann-Scully vortex (1.8), because $J(r)=1+1 / r^{2}$ in that case, and a direct calculation that can be found in Section 6.7 below reveals that assumption $\mathrm{H} 2$ also holds for the Lamb-Oseen vortex (1.9). A quantity corresponding to (1.20) appears in the work of G.I. Taylor [26] on the stability of stratified shear flows; in that context it is called the local Richardson number (see e.g. [7, Chapter 6]). Its relevance for stability was confirmed by Miles [18] and Howard [13. The ideas of Howard were translated into the columnar vortex framework by Howard and Gupta [14, where the quantity (1.20) is also shown to play an important role in the stability analysis for perturbations with nonzero angular Fourier mode $m$ and nonzero vertical wave number $k$. Indeed, it is proved in [14 that the linear operator $L_{m, k}$ in (1.15) has no unstable eigenvalue if

$$
\frac{k^{2}}{m^{2}} J(r) \geq \frac{1}{4}, \quad \text { for all } r>0,
$$

see also Proposition 3.4 below. Note that, in the case of the Lamb-Oseen vortex, inequality (1.21) is always violated for large $r>0$ because $J(r) \rightarrow 0$ as $r \rightarrow \infty$, whereas (1.21) holds for the Kaufmann-Scully vortex if and only if $m^{2} \leq 4 k^{2}$. Although Howard and Gupta's result alone is not sufficient, it plays a crucial role in our stability analysis in Section 4 , where we have to distinguish two spatial regions according to whether the local Richardson number $\left(k^{2} / \mathrm{m}^{2}\right) J(r)$ is greater or smaller than $1 / 4$. It turns out to be important for our approach that inequality (1.21) either holds for all $r \geq 0$, or is satisfied if and only if $r \leq r_{*}$ for some $r_{*}>0$. The only way to enforce that property for all possible values of $m$ and $k$ is to assume that the function $J$ in (1.20) is decreasing. However, there is no evidence that assumption $\mathrm{H} 2$ is more than a technical limitation, and we hope that this question will be clarified in the future.

Remark 1.2. Although this is not immediately obvious, assumption H2 implies the existence of a nonnegative number $\ell_{\infty} \geq 0$ such that

$$
\lim _{r \rightarrow \infty} r^{4} W(r)=\ell_{\infty}, \quad \lim _{r \rightarrow \infty} r^{5} W^{\prime}(r)=-4 \ell_{\infty}
$$

see Section 6.4 below. 
Next, we specify the function space in which we study the linearized operator $L_{m, k}$ defined in (1.13), (1.15). Since we used a Fourier decomposition to reduce our analysis to functions of the form (1.12), it is natural to work in $L^{2}$-based function spaces. Given $m \in \mathbb{Z}$ and $k \in \mathbb{R}$, we thus define the enstrophy space

$$
X_{m, k}=\left\{\omega \in L^{2}\left(\mathbb{R}_{+}, r \mathrm{~d} r\right)^{3} \mid \frac{1}{r} \partial_{r}\left(r \omega_{r}\right)+\frac{i m}{r} \omega_{\theta}+i k \omega_{z}=0\right\},
$$

equipped with the norm

$$
\|\omega\|_{L^{2}}^{2}=\int_{0}^{\infty}|\omega(r)|^{2} r \mathrm{~d} r, \quad \text { where } \quad|\omega|^{2}=\left|\omega_{r}\right|^{2}+\left|\omega_{\theta}\right|^{2}+\left|\omega_{z}\right|^{2} .
$$

It is not difficult to verify that the generator $L_{m, k}$ of the linearized evolution equation (1.15) defines a bounded linear operator in the space $X_{m, k}$ if $k \neq 0$, see Proposition 2.1 below. With this observation in mind, we can formulate our first main result:

Theorem 1.3. Consider a columnar vortex whose vorticity profile $W$ satisfies assumptions H1, H2 above. Given $m \in \mathbb{Z}$ and $k \neq 0$, let $L_{m, k}$ be the generator of the linearized evolution (1.15). Then the spectrum of $L_{m, k}$ in the enstrophy space $X_{m, k}$ satisfies

$$
\sigma\left(L_{m, k}\right) \subset i \mathbb{R}
$$

Remark 1.4. The proof actually shows that, under the normalization condition $W(0)=2$, $\sigma\left(L_{m, k}\right)$ consists of essential spectrum filling the closed interval $\{-i m b \mid 0 \leq b \leq 1\} \subset i \mathbb{R}$, and of a countable family of simple, purely imaginary eigenvalues that accumulate only at $-i m \in i \mathbb{R}$. These eigenvalues are well studied in the physical literature (a brief account is given in Section 1.3 below), and the corresponding eigenfunctions are referred to as Kelvin vibration modes. The main contribution of the present paper is to show that the operator $L_{m, k}$ has no eigenvalue outside the imaginary axis, if the vorticity profile $W$ satisfies assumptions $\mathrm{H} 1, \mathrm{H} 2$. It is interesting to note that this result remains valid for the Rankine vortex (1.7) which does not satisfy our hypotheses, see Section 6.2 below.

Remark 1.5. The particular case $k=0$, which corresponds to two-dimensional perturbations, is excluded in Theorem 1.3 because the function space $X_{m, k}$ is not appropriate in that situation. This is essentially due to the fact that the two-dimensional Biot-Savart law is ill-defined for vorticities in the enstrophy space. The problem can be eliminated by introducing a radial weight that ensures a faster decay of $\omega(r)$ as $r \rightarrow \infty$, or alternatively by working in the energy space as mentioned in Remark 1.7 below. However, since the two-dimensional stability of radially symmetric vortices is already well documented, we chose to ignore these technical issues and to concentrate here on the genuinely three-dimensional case $k \neq 0$, which was essentially unexplored until now.

According to Theorem 1.3 , for any $s \in \mathbb{C}$ with $\operatorname{Re}(s) \neq 0$, the resolvent operator $\left(s-L_{m, k}\right)^{-1}$ is well defined and bounded in the space $X_{m, k}$ if $m \in \mathbb{Z}$ and $k \neq 0$. Actually, one can prove that the resolvent is uniformly bounded for all $m \in \mathbb{Z}$ and for all nonzero $k$ in the one-dimensional lattice $\mathbb{Z} k_{0}$, where $k_{0}>0$ is arbitrary. Returning to the full linearized evolution (1.11), this proves spectral stability of the generator $L$ in the space

$$
\dot{L}_{\sigma, \text { per }, h}^{2}=\left\{\omega \in L^{2}\left(\mathbb{R}^{2} \times \mathbb{T}_{h}\right)^{3} \mid \operatorname{div} \omega=0, \quad \int_{0}^{h} \omega\left(x_{1}, x_{2}, x_{3}\right) \mathrm{d} x_{3}=0\right\},
$$

where $\mathbb{T}_{h}=\mathbb{R} /(\mathbb{Z} h)$ and $h=2 \pi / k_{0}$ is the vertical period. We can thus state our second main result : 
Theorem 1.6. Under the assumptions of Theorem 1.3, let $L$ denote the full linearized operator in (1.11). Then, for any $h>0$, the spectrum of $L$ in the space $\dot{L}_{\sigma, \mathrm{per}, h}^{2}$ satisfies

$$
\sigma(L)=i \mathbb{R} .
$$

Remark 1.7. The reason for restricting ourselves to functions with zero average in the vertical direction was explained in Remark 1.5. The same technical limitation prevents us from considering perturbations in the enstrophy space $L_{\sigma}^{2}\left(\mathbb{R}^{3}\right)$, without assuming periodicity in the vertical direction, because in that case all values of the vertical wave number $k \in \mathbb{R}$ have to be taken into account. In a subsequent work [1], we use Theorem 1.3 to obtain the equivalent of Theorem 1.6 for the Euler equation in velocity formulation. There we consider perturbations in the energy space, and we also obtain semigroup estimates for the linearized operator at a columnar vortex.

In the proof of Theorems 1.3 and 1.6, we find it convenient to normalize our velocity and vorticity profiles so that $\Omega(0)=1$ and $W(0)=2$. This leads to the following definition:

Definition 1.8. We denote by $\mathscr{W}$ the class of all vorticity profiles $W: \overline{\mathbb{R}}_{+} \rightarrow \mathbb{R}_{+}$satisfying the assumptions H1, H2 above, as well as the normalizing condition $W(0)=2$.

It is worth emphasizing here that assumption $\mathrm{H} 2$ involves the function $J$ defined in (1.20), which depends nonlinearly on the vorticity profile $W$. As a consequence, our family of admissible profiles is not a vector space, and the class $\mathscr{W}$ introduced in Definition 1.8 is not even a convex set. However, we shall prove in Section 6.4 that any profile $W \in \mathscr{W}$ is entirely determined by the auxiliary function

$$
Q(r)=\frac{1}{\sqrt{1+J(r)}}, \quad r>0,
$$

and that the class $\mathscr{W}$ can be described by simple linear constraints at the level of the function $Q$. This makes it possible to perform continuous interpolation and approximation within the class $\mathscr{W}$, and such tools will play a crucial role in the proof of Theorem 1.3 .

Remark 1.9. If we equip the class $\mathscr{W}$ with the topology of $\mathcal{C}_{b}^{1}\left(\overline{\mathbb{R}}_{+}\right)$, the Banach space of all bounded continuously differentiable functions on $\overline{\mathbb{R}}_{+}$with bounded derivative, it is easily verified that the linear operator operator $L_{m, k} \in \mathcal{L}\left(X_{m, k}\right)$ depends continuously on the vorticity profile $W \in \mathscr{W}$, see Lemma 4.1 below. In particular, isolated eigenvalues of $L_{m, k}$ outside the imaginary axis (if they are any) vary continuously when $W$ is perturbed in that topology. This implies that the conclusion (1.24) of Theorem 1.3 remains valid for any vorticity profile that belongs to the closure of the class $\mathscr{W}$ in $\mathcal{C}_{b}^{1}\left(\overline{\mathbb{R}}_{+}\right)$. This larger class contains vorticities $W$ that are not strictly decreasing functions of the radius $r$, and may even be compactly supported.

\subsection{Previous results and perspectives}

The first historical contribution regarding the stability of columnar vortices in incompressible fluids is of course the seminal work [27] by Kelvin. In that study, the focus is put on neutral modes, namely eigenmodes of the linearized Euler equation that correspond to purely imaginary eigenvalues; these were later termed "Kelvin vibration modes". As Kelvin expresses it: "The problem thus solved is the finding of the periodic disturbance in the motion of rotating liquid [...]". The computations in [27] are performed in situations where the underlying axisymmetric flow has piecewise constant vorticity; this exactly corresponds to what was called the Rankine vortex in Section 1.1 above. However, Kelvin waves are observed to play an important role in the dynamics of the Euler equation for a much wider variety of profiles, and were actively studied in the literature since then (in most cases numerically, or using asymptotic expansions combined 
with physical arguments). In the case of the Lamb-Oseen vortex, important contributions were made in particular by Le Dizès and Lacaze [16] and Fabre, Sipp and Jacquin [9], both in the inviscid case and in the vanishing viscosity limit. Unlike Kelvin (who had no computer account!), the authors of [16, 9] also consider the possibility of eigenvalues off the imaginary axis. One of the conclusions of [9] based on their numerical findings is that "[...] no amplified modes were found, a result which demonstrates the stability of the Lamb-Oseen vortex."

In a different direction, Rayleigh [20, 21] initiated the study of necessary conditions for columnar vortex instability 1 . Although it may certainly be found physically convincing, the original argument [21] leading to Rayleigh's criterion cannot be easily transposed into rigorous mathematical terms. Instead, the approach followed by Howard and Gupta [14, which we consider one of the most interesting and important contributions so far, is both rigorous and elementary. This remarkable work contains most importantly a non-conclusive but enlightening section called "Remarks on the non-axisymmetric case", in which the partial stability criterion (1.21) can be found. The authors write: "The overall conclusion of this consideration of the nonaxisymmetric case is thus essentially negative: the methods used to derive the Richardson number and semicircle results in the axisymmetric case reproduce the known results of Rayleigh for twodimensional perturbations and pure axial flow, but seem to give very little more. In fact the present situation with regard to non-axisymmetric perturbations seems to be very unsatisfactory from a theoretical point of view."

Attempts have been made to derive necessary conditions for instability extending Rayleigh's criterion to non-axisymmetric perturbations. One such criterion was proposed by Billant and Gallaire [5], following earlier work by Leibovich and Stewartson [17], and applies in a given Fourier sector. It is relatively simple to state but requires a number of a posteriori checks which could be more difficult to perform. As the authors mention, in all the situations they tested the most unstable modes were always the axisymmetric ones (this is reminiscent of Squire's theorem in the context of viscous shear flows), and therefore, in practice, Rayleigh's criterion appears to be sufficient to detect potential instabilities. Yet, a priori estimates on the possible growth in a given Fourier sector are certainly interesting per se.

Spectral stability of course does not imply stability of the flow for a Hamiltonian system such as Eq. (1.1). In a celebrated paper [3, 4], Arnold derived a nonlinear stability criterion for stationary solutions of the Euler equations, which are viewed as critical points of the kinetic energy functional over the manifold of isovortical vector fields, and he treated in detail the case of 2D flows. His approach was subsequently extended by Szeri and Holmes [25] and applied to axisymmetric perturbations of columnar vortices. A few years later, Rouchon [22] proved that the conditions in Arnold's criterion are never satisfied if one considers genuinely 3D perturbations of nontrivial stationary flows. An intermediate step between spectral and nonlinear stability is linear stability, which consists in controling the growth of the semigroup generated by the linearized operator in Theorem 1.6. Preliminary results in that direction can be found in the subsequent work [11].

We close this section mentioning that a number of interesting phenomena are known to arise, as far as instabilities are concerned, when the base flow possesses an additional axial component. Some of the works already quoted, and many others, do consider that situation as well. Since we did not investigate it at all in this work, we keep that discussion for another occasion.

\footnotetext{
${ }^{1}$ Or equivalently sufficient conditions for their stability; in the present work stability is only understood in the spectral sense, meaning the absence of eigenvalues with positive real part.
} 


\subsection{Organization of the paper}

Our strategy to prove Theorems 1.3 and 1.6 can be explained as follows. In a first step, we show in Section 2 that the essential spectrum of the operator $L_{m, k}$ is purely imaginary. The rest of the spectrum consists of isolated eigenvalues with finite multiplicity, and the corresponding eigenfunctions are solutions of a second order differential equation involving a complex potential that depends on $m, k$, and the spectral parameter $s$. The eigenvalue equation is difficult to study in general, but using techniques that date back to Rayleigh [20, 21] it is easy to verify that it has no nontrivial solution with $\operatorname{Re}(s) \neq 0$ when the perturbations are either axisymmetric $(m=0)$ or two-dimensional $(k=0)$. In Section 3 , we establish a few preliminary results in the case were $m \neq 0$ and $k \neq 0$. In particular, we derive useful identities satisfied by any nontrivial eigenfunction, and we recover the stability criterion (1.21) of Howard and Gupta. The core of the proof of Theorem 1.3 is Section 4 . We construct a suitable homotopy between the vorticity profile $W \in \mathscr{W}$ and a reference profile for which stability in the corresponding Fourier sector $X_{m, k}$ is known by Howard and Gupta's criterion. By a continuity argument, this strategy allows us to reduce the problem to proving the absence of unstable eigenvalues arbitrarily close to the imaginary axis, for a one-parameter family of profiles in the class $\mathscr{W}$. A delicate combination of integral identities and comparison arguments relying on assumption $\mathrm{H} 2$ are then used to perform such a "critical layer analysis" and hence to preclude the existence of unstable eigenvalues in the large. Finally, in Section 5, we prove uniform resolvent estimates for the linear operator $L_{m, k}$ outside the imaginary axis, which imply that the full linearization $L$ has indeed no spectrum in

that region when acting on the space $\dot{L}_{\sigma, \mathrm{per}, h}^{2}$ for any $h>0$. This is precisely the conclusion of Theorem 1.6. The last section is an appendix were several auxiliary results are established. In particular, we give useful estimates for the Biot-Savart law in the Fourier sector indexed by $m, k$, we prove the stability of Rankine's vortex (1.7) which is not covered by Theorem 1.3, and we explain how to perform continuous interpolation and approximation in the nonlinear class $\mathscr{W}$.

Acknowledgements. The authors were partially supported by grants ANR-13-BS01-0003-01 (Th.G.) and ANR-14-CE25-0009-01 (D.S.) from the "Agence Nationale de la Recherche". They benefited from discussions with S. Le Dizès, in particular during the meeting "Vortex et solitons pour les fluides classiques et quantiques" (CIRM, Marseille, 2012) where this work was initiated, and also from insightful remarks from an anonymous referee.

\section{Formulation of the spectral problem}

Let $W$ be a vorticity profile in the class $\mathscr{W}$, and let $\Omega$ be the corresponding angular velocity defined by (1.16). For a fixed value of the angular Fourier mode $m \in \mathbb{Z}$ and of the vertical wave number $k \in \mathbb{R}$, we consider the linear operator $L_{m, k}$ introduced in (1.15). In view of (1.13), we have the natural decomposition

$$
L_{m, k}=A_{m}+B_{m, k},
$$

where $A_{m}$ is the multiplication operator defined by

$$
A_{m} \omega=-i m \Omega(r) \omega+r \Omega^{\prime}(r) \omega_{r} e_{\theta},
$$

and $B_{m, k}$ is the following nonlocal perturbation:

$$
B_{m, k} \omega=i k W(r) u-W^{\prime}(r) u_{r} e_{z} .
$$

Here $u=\left(u_{r}, u_{\theta}, u_{z}\right)$ denotes the velocity obtained from the vorticity $\omega=\left(\omega_{r}, \omega_{\theta}, \omega_{z}\right)$ by solving the linear PDE system (1.14) with appropriate boundary conditions. We refer the reader to 
Section 6.1 below for a discussion of the map $\omega \mapsto u$, which we call the Biot-Savart law in the Fourier subspace indexed by $m$ and $k$. Our main goal in this paper is to study the spectral properties of the operator $L_{m, k}$ acting on the enstrophy space $X_{m, k}$ defined by (1.23).

The following simple result is the starting point of our analysis.

Proposition 2.1. Fix $m \in \mathbb{Z}$ and $k \in \mathbb{R} \backslash\{0\}$.

1) The linear operator $A_{m}$ defined by (2.2) is bounded in $X_{m, k}$ with spectrum given by

$$
\sigma\left(A_{m}\right)=\{z \in \mathbb{C} \mid z=-i m b \text { for some } b \in[0,1]\} .
$$

This spectrum is purely continuous if $m \neq 0$, and reduces to a single eigenvalue if $m=0$.

2) The linear operator $B_{m, k}$ defined by (2.3) is compact in $X_{m, k}$.

Proof. Given $s \in \mathbb{C}$ and $f=\left(f_{r}, f_{\theta}, f_{z}\right) \in X_{m, k}$, the resolvent equation $\left(s-A_{m}\right) \omega=f$ is equivalent to the linear system

$$
(s+i m \Omega(r)) \omega_{r}=f_{r}, \quad(s+i m \Omega(r)) \omega_{\theta}=f_{\theta}+r \Omega^{\prime}(r) \omega_{r}, \quad(s+i m \Omega(r)) \omega_{z}=f_{z} .
$$

As $W \in \mathscr{W}$, we know that $\Omega:[0, \infty) \rightarrow \mathbb{R}_{+}$is strictly decreasing with $\Omega(0)=1$ and $\Omega(r) \rightarrow 0$ as $r \rightarrow \infty$. Thus, if $s \neq-i m b$ for all $b \in[0,1]$, the quantity $|s+i m \Omega(r)|$ is bounded away from zero, and it follows that system (2.5) has a unique solution $\omega \in X_{m, k}$ satisfying $\|\omega\|_{L^{2}} \leq C(s)\|f\|_{L^{2}}$. On the other hand, if $m \neq 0$ and $s=-i m b$ for some $b \in[0,1]$, it is easy to verify that the operator $s-A_{m}$ is one-to-one but not onto (its range is dense but strictly contained in $X_{m, k}$ ), so that $s$ belongs to the continuous spectrum of $A_{m}$. Finally, if $m=0$, it is clear that $s=0$ is an eigenvalue of $A_{m}$, with infinite multiplicity. This proves the first part.

We next consider the operator $B_{m, k}$. If $\omega \in X_{m, k}$ and $\|\omega\|_{L^{2}} \leq 1$, Proposition 6.1 shows that the associated velocity field $u$ satisfies $\left\|\partial_{r} u\right\|_{L^{2}}+\|k u\|_{L^{2}} \leq C$ for some universal constant $C>0$. This gives a uniform bound on $u$ in $H^{1}\left(\mathbb{R}_{+}, r \mathrm{~d} r\right)$ since we assume that $k \neq 0$. By the Fréchet-Kolmogorov theorem, we deduce that the map $\omega \mapsto B_{m, k} \omega=i k W(r) u-W^{\prime}(r) u_{r} e_{z}$ is compact in $X_{m, k}$, because the functions $W$ and $W^{\prime}$ are bounded and converge to zero as $r \rightarrow \infty$.

Proposition 2.1 shows in particular that, for any $m \in \mathbb{Z}$ and any $k \in \mathbb{R} \backslash\{0\}$, the linearization $L_{m, k}=A_{m}+B_{m, k}$ defines a bounded operator in the space $X_{m, k}$. Moreover, as $B_{m, k}$ is compact, the essential spectrum of $L_{m, k}$ is the same as the (essential) spectrum of $A_{m}$, namely the closed interval $I_{m}=\{-i m b \mid 0 \leq b \leq 1\} \subset i \mathbb{R}$, see [8, Theorem I.4.1]. Note that, in the present case, the various definitions of the essential spectrum listed in [8, Section I.4] all coincide. This implies that the spectrum of $L_{m, k}$ outside the interval $I_{m}$ entirely consists of isolated eigenvalues with finite multiplicities, which can accumulate only on the essential spectrum. The proof of Theorem 1.3 is thus reduced to showing that all isolated eigenvalues of $L_{m, k}$ actually lie on the imaginary axis.

Remark 2.2. As the functions $\Omega, W$ are real-valued, it is not difficult to verify, using the definitions (2.2), (2.3) and the relations (1.14) between $u$ and $\omega$, that the spectrum of $L_{m, k}$ in $X_{m, k}$ has the following symmetries:

$$
\sigma\left(L_{m, k}\right)=\sigma\left(L_{m,-k}\right)=-\sigma\left(L_{-m, k}\right), \quad \text { and } \quad \sigma\left(L_{m, k}\right)=-\overline{\sigma\left(L_{m, k}\right)} .
$$

The corresponding mappings between eigenspaces are also easy to establish. In particular, the last relation in (2.6) means that the spectrum of $\sigma\left(L_{m, k}\right)$ is symmetric with respect to the imaginary axis, a property that will be used later on. 
As a first step in the proof of Theorem 1.3, we derive an equation for the eigenfunctions of the operator $L_{m, k}$ corresponding to eigenvalues outside the essential spectrum. In what follows, we thus assume that $s \in \mathbb{C}$ is an isolated eigenvalue of $L_{m, k}$ with eigenfunction $\omega=\left(\omega_{r}, \omega_{\theta}, \omega_{z}\right) \in$ $X_{m, k}$, and we denote by $u=\left(u_{r}, u_{\theta}, u_{z}\right)$ the velocity field associated with $\omega$ via the Biot-Savart law, see Section 6.1. As in [7, we define

$$
\gamma(r)=s+i m \Omega(r), \quad r>0 .
$$

Since $s$ does not belong to the essential spectrum of $L_{m, k}$ by assumption, it follows from Proposition 2.1 that $\gamma(r) \neq 0$ for all $r>0$.

In view of (1.13), the eigenvalue equation reads

$$
\begin{aligned}
& \gamma(r) \omega_{r}=i k W(r) u_{r}, \\
& \gamma(r) \omega_{\theta}=i k W(r) u_{\theta}+r \Omega^{\prime}(r) \omega_{r}, \\
& \gamma(r) \omega_{z}=i k W(r) u_{z}-W^{\prime}(r) u_{r}
\end{aligned}
$$

where $r \Omega^{\prime}(r)=W(r)-2 \Omega(r)$ by (1.6). If we express the vorticity $\omega$ in terms of $u$ using the relations (1.14), we obtain the equivalent system

$$
\begin{aligned}
i k W(r) u_{r}+i k \gamma(r) u_{\theta}-\frac{i m \gamma(r)}{r} u_{z} & =0, \\
i k \gamma(r) u_{r}-2 i k \Omega(r) u_{\theta}-\partial_{r}\left(\gamma(r) u_{z}\right) & =0, \\
\left(W^{\prime}(r)-\frac{i m \gamma(r)}{r}\right) u_{r}+\gamma(r) \frac{1}{r} \partial_{r}\left(r u_{\theta}\right)-i k W(r) u_{z} & =0 .
\end{aligned}
$$

Assuming for the moment that $k \neq 0$, it is straightforward to verify that the relations (2.9) $-(2.11)$ together imply the incompressibility condition

$$
\frac{1}{r} \partial_{r}\left(r u_{r}\right)+\frac{i m}{r} u_{\theta}+i k u_{z}=0 .
$$

To reduce system (2.9)-(2.12) to a single equation, we first express the azimuthal velocity $u_{\theta}$ in terms of $u_{r}, u_{z}$ using (2.9), and replace it into (2.10), (2.12) to obtain the $2 \times 2$ system

$$
\begin{aligned}
& \left(\partial_{r}^{*}-\frac{i m W(r)}{r \gamma(r)}\right) u_{r}+i k\left(1+\frac{m^{2}}{k^{2} r^{2}}\right) u_{z}=0, \\
& \left(\partial_{r}+\frac{i m W(r)}{r \gamma(r)}\right) u_{z}-i k\left(1+\frac{\Phi(r)}{\gamma(r)^{2}}\right) u_{r}=0,
\end{aligned}
$$

where $\Phi=2 \Omega W$ is the Rayleigh function and $\partial_{r}^{*}=\partial_{r}+\frac{1}{r}$. Next, observing that the coefficient of $u_{z}$ in (2.13) does note vanish, we can divide (2.13) by that coefficient and apply the differential operator $\partial_{r}+\frac{i m W}{r \gamma}$ to obtain, with the help of (2.14), the following second-order differential equation for the radial velocity:

$$
\left(\partial_{r}+\frac{i m W(r)}{r \gamma(r)}\right) \frac{r^{2}}{m^{2}+k^{2} r^{2}}\left(\partial_{r}^{*}-\frac{i m W(r)}{r \gamma(r)}\right) u_{r}=\left(1+\frac{\Phi(r)}{\gamma(r)^{2}}\right) u_{r} .
$$

If we expand the product in the left-hand side, we find after straightforward calculations

$$
-\partial_{r}\left(\frac{r^{2} \partial_{r}^{*} u_{r}}{m^{2}+k^{2} r^{2}}\right)+\left\{1+\frac{1}{\gamma(r)^{2}} \frac{k^{2} r^{2} \Phi(r)}{m^{2}+k^{2} r^{2}}+\frac{i m r}{\gamma(r)} \partial_{r}\left(\frac{W(r)}{m^{2}+k^{2} r^{2}}\right)\right\} u_{r}=0,
$$

see also [7, Eq. (15.26)]. This is the desired eigenvalue equation, which will be our main concern in the rest of this paper. It is formulated in terms of the radial velocity $u_{r}$, which satisfies $u_{r} \in H^{1}\left(\mathbb{R}_{+}, r \mathrm{~d} r\right)$ according to Proposition 6.1. In fact, we also have $u_{r} \in H_{\text {loc }}^{2}\left(\mathbb{R}_{+}\right)$in view of the divergence-free condition (2.12). 
Remark 2.3. In the case where $k=0$, a much simpler calculation shows that the eigenvalue equation is still given by (2.16) if $m \neq 0$, although the derivation above is not correct. If $k=m=0$, equation (2.16) is of course meaningless, but in that case it is obvious that system (2.8) has no nontrivial solution for $s \neq 0$.

Summarizing the arguments developed so far, the proof of Theorem 1.3 can be reduced to showing that, for all $m \in \mathbb{Z}$ and all $k \in \mathbb{R} \backslash\{0\}$, the eigenvalue equation (2.16) has no nontrivial solution $u_{r} \in H^{1}\left(\mathbb{R}_{+}, r \mathrm{~d} r\right)$ if the spectral parameter $s \in \mathbb{C}$ satisfies $\operatorname{Re}(s) \neq 0$. This is a difficult task in general, which we postpone to Sections 3 and 4 . For the time being, we just mention two important particular cases which are relatively easy to handle.

\subsection{The axisymmetric case}

In the axisymmetric case $m=0$, Proposition 2.1 asserts that the essential spectrum of $L_{0, k}$ is reduced to zero, and therefore away from the origin there may only exist eigenvalues with finite multiplicity. The spectral function (2.7) is constant in that case, and the stability equation (2.16) reduces to

$$
-\partial_{r} \partial_{r}^{*} u_{r}+k^{2}\left(1+\frac{\Phi(r)}{s^{2}}\right) u_{r}=0 .
$$

The following classical result dates back to the work of L. Rayleigh [21], and is reproduced here for the reader's convenience.

Proposition 2.4. Assume that the Rayleigh function $\Phi$ is nonnegative. Then the eigenvalue equation (2.17) has no nontrivial solution $u_{r} \in H^{1}\left(\mathbb{R}_{+}, r \mathrm{~d} r\right)$ if $\operatorname{Re}(s) \neq 0$.

Proof. According to Remark 2.3, we can suppose that $k \neq 0$. Assume that $u_{r} \in H^{1}\left(\mathbb{R}_{+}, r \mathrm{~d} r\right)$ is a nontrivial solution of (2.17) for some $s \in \mathbb{C} \backslash\{0\}$. Multiplying both sides of (2.17) by $r \bar{u}_{r}$ and integrating the resulting expression over $\mathbb{R}_{+}$, we obtain the useful relation

$$
\int_{0}^{\infty}\left\{\left|\partial_{r}^{*} u_{r}\right|^{2}+k^{2}\left(1+\frac{\Phi(r)}{s^{2}}\right)\left|u_{r}\right|^{2}\right\} r \mathrm{~d} r=0 .
$$

By assumption we have $\int_{0}^{\infty} \Phi\left|u_{r}\right|^{2} r \mathrm{~d} r>0$, because $u_{r}$ is a nontrivial solution of (2.17) and $\Phi$ is a nonnegative function with $\Phi(0)>0$. Thus taking the imaginary part of (2.18) we deduce that $\operatorname{Im}\left(s^{2}\right)=0$, hence $s \in \mathbb{R}$ or $s \in i \mathbb{R}$. The first possibility is excluded by taking the real part of (2.18), hence we conclude that $s \in i \mathbb{R}$.

Remark 2.5. Actually it was observed by Synge [24] that the Rayleigh stability criterion $\Phi \geq 0$ is not only sufficient, but also necessary in the axisymmetric case. Indeed, we know that $\Phi(0)=W(0)^{2}>0$, and for localized vortices we always have $\Phi(r) \rightarrow 0$ as $r \rightarrow \infty$. Now, assume that $\Phi(\bar{r})<0$ for some $\bar{r}>0$, and consider the Schrödinger equation

$$
-s^{2} \partial_{r} \partial_{r}^{*} u_{r}+k^{2}\left(s^{2}+\Phi(r)\right) u_{r}=E u_{r}, \quad r>0,
$$

in the semiclassical limit where $0<s \ll 1$. As the potential term $s^{2}+\Phi(r)$ takes negative values near $r=\bar{r}$, it is well known that the operator in (2.19) has negative eigenvalues $E$ if $s>0$ is sufficiently small, see e.g. [23, 12]. In fact, the number of negative eigenvalues increases unboundedly as $s \rightarrow 0$, and this implies by continuity that Eq. (2.19) with $E=0$, or equivalently Eq. (2.17), has a nontrivial solution $u_{r} \in H^{1}\left(\mathbb{R}_{+}, r \mathrm{~d} r\right)$ for a sequence of values of $s>0$ that converges to zero.

We also note that the equivalent of Synge's observation, but used for $s \in i \mathbb{R}$ instead of $s \in \mathbb{R}$, implies in contrast that, when the Rayleigh function is nonnegative, the linearized operator $L_{0, k}$ does possess nonzero eigenvalues on the imaginary axis, which correspond to Kelvin modes. 


\subsection{The two-dimensional case}

Although it is not included in Theorem 1.3, the two-dimensional case $k=0$ is worth mentioning too. When $m \neq 0$, the eigenvalue equation (2.16) reduces to

$$
-\partial_{r}\left(r^{2} \partial_{r}^{*} u_{r}\right)+\left(m^{2}+\frac{i m r W^{\prime}(r)}{\gamma(r)}\right) u_{r}=0
$$

A well-known sufficient condition for stability is that the vorticity profile $W$ be a monotone function, see e.g. [19], but unlike in the axisymmetric case no sharp criterion has been established so far. Again, for the reader's convenience, we reproduce here the easy argument showing spectral stability if $W^{\prime}$ has a constant sign.

Proposition 2.6. Assume that the vorticity profile $W$ is monotone. Then the eigenvalue equation (2.20) has no nontrivial solution $u_{r} \in H^{1}\left(\mathbb{R}_{+}, r \mathrm{~d} r\right)$ if $\operatorname{Re}(s) \neq 0$.

Proof. Assume that $u_{r} \in H^{1}\left(\mathbb{R}_{+}, r \mathrm{~d} r\right)$ is a nontrivial solution of (2.20) for some $s \in \mathbb{C}$ with $\operatorname{Re}(s) \neq 0$. Multiplying both members of $(2.20)$ by $r \bar{u}_{r}$ and integrating over $\mathbb{R}_{+}$, we obtain the relation

$$
\int_{0}^{\infty}\left\{\left|\partial_{r}\left(r u_{r}\right)\right|^{2}+\left(m^{2}+\frac{i m r W^{\prime}(r)}{\gamma(r)}\right)\left|u_{r}\right|^{2}\right\} r \mathrm{~d} r=0 .
$$

In particular, taking the imaginary part and using (2.7), we find

$$
m \operatorname{Re}(s) \int_{0}^{\infty} \frac{W^{\prime}(r)}{|\gamma(r)|^{2}}\left|u_{r}\right|^{2} r^{2} \mathrm{~d} r=0
$$

and since $W$ is monotone we conclude that $u_{r}$ is supported in the set where $W^{\prime}$ vanishes. This is clearly impossible if $W$ is not identically constant, because $u_{r}$ is a nontrivial solution of the second order ODE (2.20). But if $W$ is a constant, equation (2.21) immediately gives the desired contradiction.

\section{The eigenvalue equation for $m \neq 0$ and $k \neq 0$}

In this section we begin our study of the eigenvalue equation (2.16) in the general case where $m \neq 0$ and $k \neq 0$. In view of the symmetries (2.6), we can assume without loss of generality that $m \geq 1$ and $k>0$. We write the spectral parameter as $s=m(a-i b)$, where $a, b \in \mathbb{R}$, and we decompose

$$
\gamma(r)=s+i m \Omega(r)=i m \gamma_{\star}(r), \quad \text { where } \quad \gamma_{\star}(r)=\Omega(r)-b-i a .
$$

According to Proposition 2.1. the essential spectrum of the operator $L_{m, k}$ is the set of all $s=m(a-i b)$ such that $a=0$ and $b \in[0,1]$. Outside that set, the function $\gamma_{\star}$ is bounded away from zero for all $r>0$ and the eigenvalue equation (2.16) becomes

$$
-\partial_{r}\left(\mathcal{A}(r) \partial_{r}^{*} u_{r}\right)+\mathcal{B}(r) u_{r}=0
$$

where $\partial_{r}^{*}=\partial_{r}+\frac{1}{r}$ and

$$
\mathcal{A}(r)=\frac{r^{2}}{m^{2}+k^{2} r^{2}}, \quad \mathcal{B}(r)=1-\frac{k^{2}}{m^{2}} \frac{\mathcal{A}(r) \Phi(r)}{\gamma_{\star}(r)^{2}}+\frac{r}{\gamma_{\star}(r)} \partial_{r}\left(\frac{W(r)}{m^{2}+k^{2} r^{2}}\right) .
$$




\subsection{Asymptotic behavior at the origin and at infinity}

Our first goal is to determine the asymptotic behavior of the solutions of the complex ODE (3.2) as $r \rightarrow 0$ and $r \rightarrow \infty$, assuming that $a \neq 0$ or $b \notin[0,1]$. We start with the behavior at the origin. If $u_{r}$ is a solution of (3.2), we set

$$
u_{r}(r)=\frac{1}{r} v\left(\log \frac{1}{r}\right), \quad r>0,
$$

or equivalently $v(x)=e^{-x} u_{r}\left(e^{-x}\right)$ for $x=\log (1 / r) \in \mathbb{R}$. The new function $v: \mathbb{R} \rightarrow \mathbb{R}$ satisfies the equation

$$
v^{\prime \prime}(x)+2 k^{2} \mathcal{A}\left(e^{-x}\right) v^{\prime}(x)-\mathcal{C}(x) v(x)=0, \quad \text { where } \quad \mathcal{C}(x)=e^{-2 x} \frac{\mathcal{B}\left(e^{-x}\right)}{\mathcal{A}\left(e^{-x}\right)} .
$$

In view of (3.3) we have $\mathcal{A}\left(e^{-x}\right)=\mathcal{O}\left(e^{-2 x}\right)$ and $\mathcal{C}(x)=m^{2}+\mathcal{O}\left(e^{-2 x}\right)+\mathcal{O}\left(e^{-x}\left|W^{\prime}\left(e^{-x}\right)\right|\right)$ as $x \rightarrow+\infty$. Thus applying e.g. [6, Theorem 3.8.1], we deduce that equation (3.4) as a unique solution $v$ such that $e^{m x} v(x) \rightarrow 1$ as $x \rightarrow+\infty$. Returning to the original variables, we conclude that equation (3.2) has a unique solution $u_{r}$ such that $r^{1-m} u_{r}(r) \rightarrow 1$ as $r \rightarrow 0$. This solution $u_{r}$ and its first derivative $u_{r}^{\prime}$ depend continuously on the various parameters in (3.2), including the vorticity profile $W \in \mathcal{C}_{b}^{1}\left(\overline{\mathbb{R}}_{+}\right)$and the spectral parameter $s=m(a-i b) \in \mathbb{C}$, uniformly in $r$ on any bounded interval of the form $(0, R)$. Any linearly independent solution of (3.2) blows up like $r^{-1-m}$ as $r \rightarrow 0$, and is therefore not square integrable near the origin.

We next study the behavior at infinity. If $u_{r}$ is a solution of (3.2), we define $w(r)=r^{1 / 2} u_{r}(r)$ and obtain for $w$ the equation

$$
w^{\prime \prime}(r)+\frac{\mathcal{A}^{\prime}(r)}{\mathcal{A}(r)} w^{\prime}(r)-\mathcal{D}(r) w(r)=0, \quad \text { where } \quad \mathcal{D}(r)=\frac{\mathcal{B}(r)}{\mathcal{A}(r)}+\frac{3}{4 r^{2}}-\frac{1}{2 r} \frac{\mathcal{A}^{\prime}(r)}{\mathcal{A}(r)} .
$$

We have $\mathcal{A}^{\prime}(r) / \mathcal{A}(r)=\mathcal{O}\left(r^{-3}\right)$ and $\mathcal{D}(r)=k^{2}+\mathcal{O}\left(r^{-2}\right)$ as $r \rightarrow \infty$, because Remark 1.2 implies that $W(r)=\mathcal{O}\left(r^{-4}\right), W^{\prime}(r)=\mathcal{O}\left(r^{-5}\right)$, and $\Phi(r)=\mathcal{O}\left(r^{-6}\right)$ in that limit. Invoking again $[6$, Theorem 3.8.1], we deduce that (3.5) has a unique solution $w$ such that $e^{k r} w(r) \rightarrow 1$ as $r \rightarrow \infty$, hence (3.2) has a unique solution satisfying $r^{1 / 2} e^{k r} u_{r}(r) \rightarrow 1$ as $r \rightarrow \infty$. This solution and its first derivative depend continuously on the parameters in (3.2), uniformly on the interval $(R, \infty)$ for any $R>0$. Any linearly independent solution of (3.2) grows like $r^{-1 / 2} e^{k r}$ as $r \rightarrow \infty$, and is therefore not square integrable.

Summarizing, we have shown:

Lemma 3.1. If $m \neq 0$ and $k \neq 0$, any eigenvalue of the linear operator $L_{m, k} \in \mathcal{L}\left(X_{m, k}\right)$ outside the essential spectrum (2.4) is necessarily simple. Moreover, if $u_{r}$ is the radial velocity profile of the corresponding eigenfunction, there exist $\alpha, \beta \in \mathbb{C}$ such that

$$
\lim _{r \rightarrow 0} r^{1-|m|} u_{r}(r)=\alpha, \quad \text { and } \quad \lim _{r \rightarrow \infty} r^{1 / 2} e^{|k| r} u_{r}(r)=\beta .
$$

\subsection{Eigenvalues on the imaginary axis: Kelvin waves}

In a second step, we consider the eigenvalues of the linearized operator $L_{m, k}$ on the imaginary axis. The corresponding eigenfunctions describe "vibration modes" of the columnar vortex, and were first studied by Kelvin [27] in the particular case of Rankine's vortex. Strictly speaking, this subsection is not part of the proof of Theorem 1.3. but in view of the physical relevance of the Kelvin waves it is worth mentioning a few results that can be rigorously established. 
In what follows, we thus assume that $a=0$ and $b \notin(0,1)$, so that $\gamma_{\star}(r) \neq 0$ for all $r>0$. In that case equation (3.2) has real coefficients, and its solutions can be studied using standard ODE techniques. For simplicity we suppose here that the vorticity profile $W \in \mathscr{W}$ is the restriction to $\mathbb{R}_{+}$of a smooth even function on $\mathbb{R}$ satisfying $W^{\prime \prime}(0)<0$, as it is the case for the KaufmannScully vortex (1.8) or the Lamb-Oseen vortex (1.9). We consider separately the regimes where $b \geq 1$ and $b \leq 0$.

Lemma 3.2. For any $m \neq 0$ and $k \neq 0$, the set of all $b>1$ such that $E q$. (3.2) with $a=0$ has $a$ nontrivial solution in $H^{1}\left(\mathbb{R}_{+}, r \mathrm{~d} r\right)$ is a countable family which accumulates only at 1 . Moreover, Eq. (3.2) has no nontrivial solution in $H^{1}\left(\mathbb{R}_{+}, r \mathrm{~d} r\right)$ if $a=0$ and $b=1$.

Proof. When $b>1$, we apply to Eq. (3.2) the change of variables $u_{r}=r^{m} \mathcal{A}(r)^{-1 / 2} v$, where $\mathcal{A}(r)$ is as in (3.3). A direct calculation shows that the new function $v$ satisfies

$$
-\partial_{r}^{2} v-\frac{2 m+1}{r} \partial_{r} v+\left(k^{2}+\mathcal{F}(r)+\mathcal{G}(r)\right) v=0, \quad r>0,
$$

where

$$
\mathcal{F}(r)=\frac{k^{2} \mathcal{A}(r)}{r^{2}}\left(-2+3 k^{2} \mathcal{A}(r)\right), \quad \mathcal{G}(r)=-\frac{k^{2}}{m^{2}} \frac{\Phi(r)}{\gamma_{\star}(r)^{2}}+\frac{r}{\mathcal{A}(r) \gamma_{\star}(r)} \partial_{r}\left(\frac{W(r)}{m^{2}+k^{2} r^{2}}\right) .
$$

We assume that $b=1+h^{2}$ for some small $h>0$, and we expand

$$
-\gamma_{\star}(r)=1+h^{2}-\Omega(r)=h^{2}+\rho r^{2}+\mathcal{O}\left(r^{4}\right), \quad \text { as } r \rightarrow 0,
$$

where $\rho=-\Omega^{\prime \prime}(0) / 2=-W^{\prime \prime}(0) / 8>0$. If $r=h s$, it is straightforward to verify that

$$
h^{4}\left(k^{2}+\mathcal{F}(h s)+\mathcal{G}(h s)\right)=-\frac{4 k^{2}}{m^{2}} \frac{1}{\left(1+\rho s^{2}\right)^{2}}+\mathcal{O}\left(h^{2}\right), \quad \text { as } h \rightarrow 0,
$$

uniformly for all $s>0$. Thus the new function $w$ defined by setting $w(s)=v(h s)$ satisfies the semi-classical Schrödinger equation

$$
\mathscr{L}_{h} w:=-h^{2}\left(\partial_{s}^{2} w+\frac{2 m+1}{s} \partial_{s} w\right)-\frac{4 k^{2}}{m^{2}} \frac{w}{\left(1+\rho s^{2}\right)^{2}}+U(s, h) w=0,
$$

for all $s>0$, where $U(s, h)=\mathcal{O}\left(h^{2}\right)$ as $h \rightarrow 0$, uniformly in $s$. Since the principal part of the potential term in (3.7) is negative, standard results in semiclassical analysis [23, 12] show that the operator $\mathscr{L}_{h}$ has negative eigenvalues if $h$ is sufficiently small, and that the number of these bound states is $\mathcal{O}\left(h^{-1}\right)$ as $h \rightarrow 0$. Moreover, as $\mathcal{F}(r)+\mathcal{G}(r) \rightarrow 0$ as $r \rightarrow \infty$, the bottom of the essential spectrum of $\mathscr{L}_{h}$ is $k^{2} h^{4}>0$ for any $h>0$. These two observations together imply that $\mathscr{L}_{h}$ has a zero eigenvalue for a countable sequence $h_{n} \rightarrow 0$, and returning to the original variables we conclude that Eq. (3.2) with $a=0$ has a nontrivial solution in $H^{1}\left(\mathbb{R}_{+}, r \mathrm{~d} r\right)$ for a sequence $b_{n}=1+h_{n}^{2} \rightarrow 1$.

When $b=1$, namely $h=0$, the leading term in the function $\mathcal{B}(r) / \mathcal{A}(r)$ satisfies

$$
\frac{k^{2}}{m^{2}} \frac{\Phi(r)}{\gamma_{\star}(r)^{2}}=\frac{\Theta^{2}}{r^{4}}\left(1+\mathcal{O}\left(r^{2}\right)\right) \quad \text { as } r \rightarrow 0, \quad \text { where } \quad \Theta^{2}=\frac{4 k^{2}}{m^{2} \rho^{2}} .
$$

To investigate the behavior of the solutions of (3.2) near $r=0$ in that case, it is useful make the change of variables $u_{r}(r)=r^{-1 / 2} U(1 / r)$. Setting $x=1 / r$, this leads to an equation of the form

$$
U^{\prime \prime}(x)+\tilde{\mathcal{C}}(1 / x) U^{\prime}(x)+\tilde{\mathcal{D}}(1 / x) U(x)=0, \quad x>0,
$$


where $\tilde{\mathcal{C}}(r)=\mathcal{O}\left(r^{3}\right)$ and $\tilde{\mathcal{D}}(r)=\Theta^{2}+\mathcal{O}\left(r^{2}\right)$ as $r \rightarrow 0$. Using [6, Theorem 3.8.1], we deduce that Eq. (3.8) has two linearly independent solutions satisfying $U_{ \pm}(x)=e^{ \pm i \Theta x}(1+\mathcal{O}(1 / x))$ as $x \rightarrow+\infty$. If we now return to the original variables, we conclude that Eq. (3.2) has two linearly independent solutions $\phi_{ \pm}$such that

$$
\phi_{ \pm}(r)=\frac{1}{\sqrt{r}} e^{ \pm i \Theta / r}(1+\mathcal{O}(r)), \quad \text { as } r \rightarrow 0 .
$$

As is easily verified, no nontrivial linear combination of $\phi_{+}$and $\phi_{-}$can belong to $H^{1}\left(\mathbb{R}_{+}, r \mathrm{~d} r\right)$, which means that Eq. (3.2) has no nontrivial solution if $a=0$ and $b=1$.

The situation is completely different when $b \leq 0$.

Lemma 3.3. For any $m \neq 0$ and $k \neq 0$, the set of all $b \leq 0$ such that Eq. (3.2) with $a=0$ has a nontrivial solution in $H^{1}\left(\mathbb{R}_{+}, r \mathrm{~d} r\right)$ is finite. Moreover:

1) This set is nonempty for a finite number of values of $m$ only;

2) For both the Kaufmann-Scully vortex (1.8) and the Lamb-Oseen vortex (1.9), Eq. (3.2) has no nontrivial solution when $a=0$ and $b \leq 0$ if $|m| \geq 2$.

Proof. If $a=0$ and $b \leq 0$, then $\gamma_{\star}(r)=\Omega(r)-b=\Omega(r)+|b|>0$. In this region, it is easy to verify that the coefficient $\mathcal{B}(r)<1$ defined in (3.3) is an increasing function of both parameters $|m|$ and $|b|$. Moreover, using the bounds on $\Omega, W$, and $\Phi$ which follow from assumptions H1, $\mathrm{H} 2$, see Remark 1.2, we obtain the following estimate:

$$
\sup _{r>0}(1-\mathcal{B}(r)) \leq \frac{C}{m^{2}} \sup _{r>0}\left(\frac{\Phi(r)}{(\Omega(r)+|b|)^{2}}+\frac{W(r)+r\left|W^{\prime}(r)\right|}{\Omega(r)+|b|}\right) \leq \frac{C}{m^{2}} \frac{1}{1+|b|},
$$

where the constant $C$ depends only on the vorticity profile. As a consequence, we see that $\mathcal{B}(r) \geq 0$ when $|m|$ or $|b|$ is large enough, and this implies that Eq. (3.2) has no nontrivial solution, see (3.10) below. It follows that the linearized operator $L_{m, k}$ can have eigenvalues $s=m(a-i b)$ with $a=0$ and $b \leq 0$ only for a finite number of values of $m \in \mathbb{Z}$, and using Sturm-Liouville theory we also conclude that, for any $m \in \mathbb{Z}$, there exist only finitely many eigenvalues with $a=0$ and $b \leq 0$. Interestingly enough, for both the Kaufmann-Scully vortex (1.8) and the Lamb-Oseen vortex (1.9), an explicit calculation which is reproduced in Section 6.7 shows that $\mathcal{B}(r) \geq 1-4 / m^{2}$, so that there are no eigenvalues in this region when $|m| \geq 2$.

As a final comment, we mention that, when $m= \pm 1$, there are always eigenvalues with $a=0$ and $b \leq 0$. Indeed, due to translation invariance, the operator $L_{m, 0}$ has a zero eigenvalue with eigenfunction

$$
u=-i m \Omega e_{r}+(W-\Omega) e_{\theta}, \quad \omega=W^{\prime} e_{z} .
$$

That eigenvalue bifurcates out of the essential spectrum as the parameter $k$ varies, so that $L_{m, k}$ has at least one eigenvalue $s=-i m b$ with $b<0$ if $|m|=1$ and $|k|$ is small enough.

\subsection{Eigenvalues outside the imaginary axis: Howard identities}

For our next step in the study of the eigenvalue equation (3.2), we use a classical method originally due to Rayleigh [20] to show that the linearized operator $L_{m, k}$ has no spectrum in large regions of the complex plane, which are depicted in Fig. 1. The idea is to derive integral identities satisfied by the hypothetical eigenfunctions, which eventually lead to a contradiction. 
Assume thus that the eigenvalue equation (3.2) has a nontrivial solution $u_{r} \in H^{1}\left(\mathbb{R}_{+}, r \mathrm{~d} r\right)$, for some $s=m(a-i b) \in \mathbb{C}$, where $a \neq 0$. Multiplying both sides of (3.2) by $r \bar{u}_{r}$ and integrating over $\mathbb{R}_{+}$, we easily obtain, using the results of Section 3.1]:

$$
\int_{0}^{\infty}\left(\mathcal{A}(r)\left|\partial_{r}^{*} u_{r}\right|^{2}+\mathcal{B}(r)\left|u_{r}\right|^{2}\right) r \mathrm{~d} r=0
$$

Note that the function $\mathcal{B}$ is complex-valued if $a \neq 0$, so that (3.10) gives two integral relations for the radial velocity $u_{r}$. For instance, taking the imaginary part of (3.10) and using the expression (3.3) of $\mathcal{B}$, we obtain the identity

$$
a \int_{0}^{\infty}\left\{\frac{2(b-\Omega(r))}{\left(a^{2}+(\Omega-b)^{2}\right)^{2}} \frac{k^{2}}{m^{2}} \mathcal{A}(r) \Phi(r)+\frac{r}{a^{2}+(\Omega-b)^{2}} \partial_{r}\left(\frac{W(r)}{m^{2}+k^{2} r^{2}}\right)\right\}\left|u_{r}\right|^{2} r \mathrm{~d} r=0 .
$$

This relation is identically satisfied if $a=0$, but gives useful information if $a \neq 0$. For instance, if $b \leq 0$, then $b-\Omega(r)<0$ for all $r>0$, and assumption H1 implies that

$$
\Phi(r)>0 \quad \text { and } \quad \partial_{r}\left(\frac{W(r)}{m^{2}+k^{2} r^{2}}\right)<0, \quad \text { for all } r>0 .
$$

Thus the integrand in (3.11) is nonpositive and not identically zero, hence equality (3.11) cannot hold. We conclude that the operator $L_{m, k}$ has no eigenvalue $s=m(a-i b)$ with $a \neq 0$ and $b \leq 0$, see Fig. 1. Unfortunately, we do not know how to use the relation (3.10) to preclude the existence of eigenvalues of $L_{m, k}$ in other regions of the complex plane.

The following approach, due to Howard [13, 14, provides other identities similar to (3.10), which give further information on the possible eigenvalues. Define $u_{r}=q(r) v_{r}$, where $q$ is a (real or complex valued) weight function satisfying $q(r) \neq 0$ for all $r>0$. Then $v_{r}$ is a solution to

$$
-\partial_{r}\left(q(r)^{2} \mathcal{A}(r) \partial_{r}^{*} v_{r}\right)+\mathcal{E}(r) v_{r}=0,
$$

where

$$
\mathcal{E}(r)=q(r)^{2} \mathcal{B}(r)-q(r) q^{\prime}(r)\left(\mathcal{A}^{\prime}(r)-\frac{\mathcal{A}(r)}{r}\right)-q(r) q^{\prime \prime}(r) \mathcal{A}(r) .
$$

Multiplying both sides of (3.12) by $r \bar{v}_{r}$ and integrating over $\mathbb{R}_{+}$, we deduce

$$
\int_{0}^{\infty}\left(q(r)^{2} \mathcal{A}(r)\left|\partial_{r}^{*} v_{r}\right|^{2}+\mathcal{E}(r)\left|v_{r}\right|^{2}\right) r \mathrm{~d} r=0 .
$$

If $q$ is real-valued, then $q^{2}\left|v_{r}\right|^{2}=\left|u_{r}\right|^{2}$ and taking the imaginary part of (3.13) we recover (3.11), but the real part gives new information. If $q$ is complex, both the real and the imaginary parts of (3.13) provide new information.

Following [14], we now consider in more detail some interesting particular cases of (3.13).

Choice 1: $q(r)=\gamma_{\star}(r)$. We then have

$$
\begin{aligned}
\mathcal{E}(r)= & \gamma_{\star}(r)^{2}-\frac{k^{2}}{m^{2}} \mathcal{A}(r) \Phi(r)+r \gamma_{\star}(r) \partial_{r}\left(\frac{W(r)}{m^{2}+k^{2} r^{2}}\right) \\
& -\gamma_{\star}(r) \gamma_{\star}^{\prime}(r)\left(\mathcal{A}^{\prime}(r)-\frac{\mathcal{A}(r)}{r}\right)-\gamma_{\star}(r) \gamma_{\star}^{\prime \prime}(r) \mathcal{A}(r) .
\end{aligned}
$$

Since $r \gamma_{\star}^{\prime}(r)=r \Omega^{\prime}(r)=W(r)-2 \Omega(r)$, we observe that

$$
\gamma_{\star}^{\prime}(r)\left(\mathcal{A}^{\prime}(r)-\frac{\mathcal{A}(r)}{r}\right)+\gamma_{\star}^{\prime \prime}(r) \mathcal{A}(r)=r \partial_{r}\left(\frac{\gamma_{\star}^{\prime}(r) \mathcal{A}(r)}{r}\right)=r \partial_{r}\left(\frac{W(r)-2 \Omega(r)}{m^{2}+k^{2} r^{2}}\right),
$$


and we deduce the following simpler expression of $\mathcal{E}(r)$ :

$$
\mathcal{E}(r)=\gamma_{\star}(r)^{2}-\frac{k^{2}}{m^{2}} \mathcal{A}(r) \Phi(r)+2 r \gamma_{\star}(r) \partial_{r}\left(\frac{\Omega(r)}{m^{2}+k^{2} r^{2}}\right) .
$$

In particular, taking the imaginary part of (3.13), we obtain the identity

$$
2 a \int_{0}^{\infty}\left\{(b-\Omega(r))\left(\mathcal{A}(r)\left|\partial_{r}^{*} v_{r}\right|^{2}+\left|v_{r}\right|^{2}\right)-r \partial_{r}\left(\frac{\Omega(r)}{m^{2}+k^{2} r^{2}}\right)\left|v_{r}\right|^{2}\right\} r \mathrm{~d} r=0 .
$$

If we now assume that $b \geq 1$, so that $b-\Omega(r)>0$ for all $r>0$, we see that all terms in the integrand of (3.15) are nonnegative, which leads to a contradiction if $a \neq 0$. We conclude that the linear operator $L_{m, k}$ has no eigenvalue $s=m(a-i b)$ if $a \neq 0$ and $b \geq 1$, see Fig. 1 .

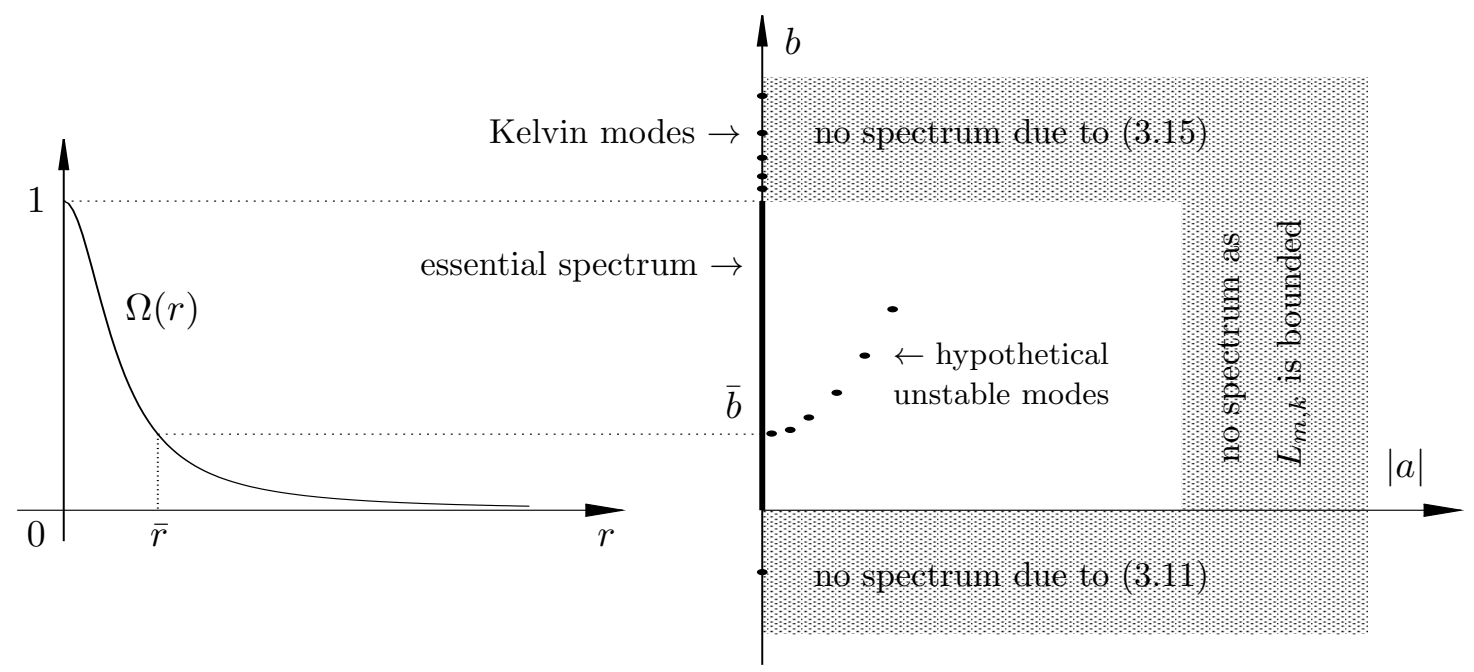

Fig. 1: (right) The information obtained so far on the spectrum of the linearized operator (2.1), using the parametrization $s=m(a-i b)$. Kelvin modes are located on the imaginary axis $a=0$, and accumulate only at the upper edge of the essential spectrum, which fills the segment $a=0, b \in[0,1]$. The rest of the spectrum, if any, consists of isolated eigenvalues which can accumulate only on the essential spectrum, and are contained in a region of the form $|a| \leq M, b \in[0,1]$ according to Proposition 3.4. (left) The angular velocity profile $\Omega$ and the critical radius $\bar{r}$ associated with a spectral point $a=0, b=\bar{b}$ inside the essential spectrum.

Choice 2: $q(r)=\gamma_{\star}(r)^{1 / 2}$. Proceeding as above, we find

$$
\begin{aligned}
\mathcal{E}(r)= & \gamma_{\star}(r)-\frac{k^{2}}{m^{2}} \frac{\mathcal{A}(r) \Phi(r)}{\gamma_{\star}(r)}+r \partial_{r}\left(\frac{W(r)}{m^{2}+k^{2} r^{2}}\right) \\
& -\frac{1}{2} \gamma_{\star}^{\prime}(r)\left(\mathcal{A}^{\prime}(r)-\frac{\mathcal{A}(r)}{r}\right)-\frac{1}{2} \gamma_{\star}^{\prime \prime}(r) \mathcal{A}(r)+\frac{1}{4} \frac{\gamma_{\star}^{\prime}(r)^{2}}{\gamma_{\star}(r)} \mathcal{A}(r) .
\end{aligned}
$$

Using again (3.14), we deduce that

$$
\mathcal{E}(r)=\gamma_{\star}(r)-\frac{k^{2}}{m^{2}} \frac{\mathcal{A}(r) \Phi(r)}{\gamma_{\star}(r)}+\frac{r}{2} \partial_{r}\left(\frac{W(r)+2 \Omega(r)}{m^{2}+k^{2} r^{2}}\right)+\frac{1}{4} \frac{\Omega^{\prime}(r)^{2}}{\gamma_{\star}(r)} \mathcal{A}(r) .
$$

In particular, taking the imaginary part of (3.13), we obtain the identity

$$
-a \int_{0}^{\infty}\left\{\mathcal{A}(r)\left|\partial_{r}^{*} v_{r}\right|^{2}+\left|v_{r}\right|^{2}+\frac{\mathcal{A}(r)}{a^{2}+(\Omega-b)^{2}}\left(\frac{k^{2} \Phi(r)}{m^{2}}-\frac{\Omega^{\prime}(r)^{2}}{4}\right)\left|v_{r}\right|^{2}\right\} r \mathrm{~d} r=0 .
$$


As a consequence, if we assume that

$$
J(r) \equiv \frac{\Phi(r)}{\Omega^{\prime}(r)^{2}} \geq \frac{m^{2}}{4 k^{2}}, \quad \text { for all } r>0,
$$

we see that all terms in the integrand of (3.16) are nonnegative, which leads to a contradiction if $a \neq 0$. We conclude that (3.17) is a sufficient condition for spectral stability. Unfortunately, condition (3.17) is never met for the Lamb-Oseen vortex, because $J(r) \rightarrow 0$ as $r \rightarrow+\infty$ in that case. In the case of the Kaufmann-Scully vortex, it is satisfied only if $m^{2} \leq 4 k^{2}$.

The results obtained by Howard's approach can thus be summarized as follows.

Proposition 3.4. Assume that the vorticity profile $W$ satisfies assumption H1 in Section 1.2. Then for any $m \neq 0$ and $k \neq 0$ the following holds:

i) The linearized operator $L_{m, k}$ has no eigenvalue $s=m(a-i b)$ with $a \neq 0$ and $b(1-b) \leq 0$.

ii) If condition (3.17) is satisfied, then $L_{m, k}$ has no eigenvalue outside the imaginary axis.

\subsection{Critical layers and embedded eigenvalues}

We assume here that $a=0$ and $0<b<1$, which means that the spectral parameter $s=m(a-i b)$ is contained in the essential spectrum of the linearized operator $L_{m, k}$, and does not coincide with one of its endpoints. The natural extension of the eigenvalue equation (3.2) to this situation 2 is

$$
-\partial_{r}\left(\mathcal{A}(r) \partial_{r}^{*} u_{r}\right)+\left[1-\frac{k^{2}}{m^{2}} \frac{\mathcal{A}(r) \Phi(r)}{(\Omega(r)-b)^{2}}+\frac{r}{(\Omega(r)-b)} \partial_{r}\left(\frac{W(r)}{m^{2}+k^{2} r^{2}}\right)\right] u_{r}=0 .
$$

Since the value of $b$ belongs to the range of the angular velocity $\Omega$, both denominators in (3.18) vanish at exactly one point $\bar{r}>0$, characterized by $\Omega(\bar{r})=b$, so that equation (3.18) becomes singular at that point. In the physical literature, singularities of the eigenvalue equation are usually avoided by allowing the variable $r$ to take slightly complex values, a procedure that is referred to as "critical layer analysis" in this context [7].

To perform such an analysis, we restrict our attention in the rest of this section to vorticity profiles $W$ which satisfy assumption $\mathrm{H} 1$ and, in addition, are real-analytic on $(0, \infty)$, so that the angular velocity $\Omega$ and the Rayleigh function $\Phi$ are analytic too. According to the usual terminology, the point $\bar{r}$ is then a regular singular point of equation (3.18), see e.g. [6, Chapter 4] or Section 6.3 below. Extending the range of the variable $r$ to a neighbourhood of $(0, \infty)$ in $\mathbb{C}$ allows us to make a connection between solutions of (3.18) defined on the interval $(0, \bar{r})$ and others defined on $(\bar{r}, \infty)$. In a neighborhood of $\bar{r}$, the behavior of the solutions of (3.18) is determined by the roots $d$ of the indicial equation

$$
d(d-1)+\frac{k^{2}}{m^{2}} J(\bar{r})=0
$$

We distinguish three cases.

Case 1: $0<J(\bar{r})<m^{2} /\left(4 k^{2}\right)$. The roots of (3.19) are real and simple:

$$
d_{ \pm}:=\frac{1}{2} \pm\left(\frac{1}{4}-\frac{k^{2}}{m^{2}} J(\bar{r})\right)^{\frac{1}{2}}
$$

\footnotetext{
${ }^{2}$ We emphasize that the derivation of (3.2) from the spectral problem was performed in Section 2 under the assumption that $s=m(a-i b)$ does not belong to the essential spectrum.
} 
In particular, we have $0<d_{-}<1 / 2<d_{+}<1$. The Frobenius method [6, Section 4.8] can be used to construct two real-valued analytic functions $V_{ \pm}$on $(0, \infty)$ such that $V_{ \pm}(\bar{r})=1$ and such that the functions $\phi_{ \pm}$defined by

$$
\phi_{ \pm}(r)=|b-\Omega(r)|^{d_{ \pm}} e^{i \frac{\pi}{2}(1-\operatorname{sgn}(b-\Omega(r))) d_{ \pm}} V_{ \pm}(r), \quad r>0,
$$

are independent solutions of (3.18) on both intervals $(0, \bar{r})$ and $(\bar{r}, \infty)$. Note that $\phi_{ \pm}$are realvalued on $(\bar{r}, \infty)$, but complex-valued (although with a constant phase) on the interval $(0, \bar{r})$.

Case 2: $J(\bar{r})>m^{2} /\left(4 k^{2}\right)$. The roots of (3.19) are complex conjugate :

$$
d_{ \pm}:=\frac{1}{2} \pm i \delta, \quad \text { where } \quad \delta=\left(\frac{k^{2}}{m^{2}} J(\bar{r})-\frac{1}{4}\right)^{\frac{1}{2}} .
$$

Similarly, the Frobenius method yields the existence of two independent solutions $\phi_{ \pm}$which we write in the form

$$
\phi_{ \pm}(r)=|b-\Omega(r)|^{1 / 2} e^{ \pm i \delta \log |b-\Omega(r)|} e^{i \frac{\pi}{2}(1-\operatorname{sgn}(b-\Omega(r))) d_{ \pm}} V_{ \pm}(r) .
$$

Case 3: $J(\bar{r})=m^{2} /\left(4 k^{2}\right)$. Equation (3.19) possesses the unique root $1 / 2$ with multiplicity two, and two independent solutions of $(\underline{3.18})$ can be constructed such that

$$
\begin{aligned}
& \phi_{+}(r)=|b-\Omega(r)|^{\frac{1}{2}} e^{i \frac{\pi}{4}(1-\operatorname{sgn}(b-\Omega(r)))} V_{+}(r), \\
& \phi_{-}(r)=|b-\Omega(r)|^{\frac{1}{2}} e^{i \frac{\pi}{4}(1-\operatorname{sgn}(b-\Omega(r)))}\left[\left(\log |b-\Omega(r)|+i \frac{\pi}{2}(1-\operatorname{sgn}(b-\Omega(r)))\right) V_{+}(r)+V_{-}(r)\right] .
\end{aligned}
$$

The following technical lemma emphasizes the relevance of the singular functions $\phi_{ \pm}$for the approximation of solutions of (3.18) by non-singular solutions of (3.2). In the statement, the vorticity profile $W$, the spectral parameter $s=-i m b$ and the corresponding singular radius $\bar{r}$ are defined as above. However, we consider a sequence $\left(u_{n}\right)_{n \in \mathbb{N}}$ of smooth solutions of the eigenvalue equation (3.2) where the spectral parameter $s$ is replaced by some complex number $s_{n}$ with nonzero real part (so that $s_{n}$ does not belong to the essential spectrum), and where also the vorticity profile $W$ is replaced by some function $W_{n}$ that is allowed to depend on $n 3$ We assume that $s_{n} \rightarrow s$ and $W_{n} \rightarrow W$ as $n \rightarrow \infty$. In what follows, for $\rho>0$ we denote by $\mathbb{D}(\bar{r}, \rho) \subset \mathbb{C}$ the open disc of radius $\rho$ centered at $\bar{r}+0 i$. When no confusion is possible, we also use the same symbols for functions of the real variable $r \in(0, \infty)$ and their analytic extensions into (part of) the complex plane.

Lemma 3.5. Let $\left(u_{n}\right)_{n \in \mathbb{N}}$ be a sequence of solutions of (3.2) corresponding to a sequence of spectral parameters $s_{n}=m\left(a_{n}-i b_{n}\right)$ and of real-valued analytic profiles $W_{n}$. Suppose that

i) $a_{n}>0$ for all $n$, and $a_{n} \rightarrow 0, b_{n} \rightarrow b \in(0,1)$ as $n \rightarrow \infty$;

ii) $W_{n} \rightarrow W$ in $\mathcal{C}_{b}^{1}\left(\overline{\mathbb{R}}_{+}\right)$as $n \rightarrow \infty$;

iii) there exists $\rho>0$ such that, for all $n \in \mathbb{N}$, the radius of analyticity of $W_{n}$ at $\bar{r}$ is at least equal to $\rho$, and $W_{n} \rightarrow W$ uniformly in $\mathbb{D}(\bar{r}, \rho)$.

If $u_{n}(r)$ and $u_{n}^{\prime}(r)$ have a limit as $n \rightarrow \infty$ for some $r \in(0, \infty) \backslash\{\bar{r}\}$, then there exist $\alpha_{ \pm} \in \mathbb{C}$ such that $u_{n} \rightarrow \alpha_{+} \phi_{+}+\alpha_{-} \phi_{-}$in the $\mathcal{C}^{1}$ topology on compact subsets of $(0, \infty) \backslash\{\bar{r}\}$, where $\phi_{ \pm}$ are given by (3.20), (3.21) or (3.22) depending on the roots of the indicial equation.

The proof of Lemma 3.5 is postponed to Section 6.3 below where we also establish the main properties of $\phi_{ \pm}$, in particular the analyticity of $V_{ \pm}$across the singularity $\bar{r}$ and the fact

\footnotetext{
${ }^{3}$ The reason for the latter will become clear in Section 4 .
} 
these functions are real-valued. For the moment, we observe that the implicit determination of logarithms we opted for in constructing the solutions $\phi_{ \pm}$is directly related to the assumption that $a_{n}>0$ in Lemma 3.5. An approximation procedure valid for negative values of $a_{n}$ would involve the complex conjugates of the functions $\phi_{ \pm}$defined in (3.20)-(3.22).

Remark 3.6. The expressions (3.20)-(3.22) show in particular that no nontrivial solution of (3.18) lies in $H^{2}(\bar{r}-\epsilon, \bar{r}+\epsilon)$ if $\epsilon>0$. As we know that the radial velocity $u_{r}$ associated with any vorticity vector $\omega \in X_{m, k}$ belongs to $H^{1}\left(\mathbb{R}_{+}, r \mathrm{~d} r\right) \cap H_{\text {loc }}^{2}\left(\mathbb{R}_{+}\right)$, we deduce from the observation above that the linear operator $L_{m, k}$ acting on $X_{m, k}$ has no nonzero eigenvalue $s$ embedded in the continuous spectrum (2.4).

Finally, repeating the proof of Lemma 3.1 for Eq. (3.18), we easily obtain

Lemma 3.7. If $m \neq 0, k \neq 0$, and $0<b<1$, there exist a unique solution $\psi_{0}$ of (3.18) on $(0, \bar{r})$ and a unique solution $\psi_{\infty}$ of (3.18) on $(\bar{r}, \infty)$ such that

$$
\lim _{r \rightarrow 0} r^{1-|m|} \psi_{0}(r)=1, \quad \text { and } \quad \lim _{r \rightarrow \infty} r^{1 / 2} e^{|k| r} \psi_{\infty}(r)=1 .
$$

Moreover, both $\psi_{0}$ and $\psi_{\infty}$ are real-valued.

Since (3.18) is a linear equation, we infer the existence of constants $\alpha_{ \pm}^{0}, \alpha_{ \pm}^{\infty} \in \mathbb{C}$ such that

$$
\psi_{0}=\alpha_{-}^{0} \phi_{-}+\alpha_{+}^{0} \phi_{+} \quad \text { on }(0, \bar{r}), \quad \psi_{\infty}=\alpha_{-}^{\infty} \phi_{-}+\alpha_{+}^{\infty} \phi_{+} \quad \text { on }(\bar{r}, \infty),
$$

where $\phi_{ \pm}$are defined in (3.20) $-(3.22)$.

\section{The homotopy argument}

This section is the core of the proof of Theorem 1.3. We concentrate on the situation where the angular Fourier mode $m$ and the vertical wave number $k$ are both nonzero, because the cases $m=0$ and $k=0$ have already been treated in Sections 2.1 and 2.2, respectively. In view of the symmetry properties (2.6), we can assume without loss of generality that $m \geq 1$ and $k>0$.

The argument is by contradiction: given a vorticity profile $W$ satisfying assumptions H1, H2 in Section 1.2, we assume that there exist an integer $m \geq 1$ and a real number $k>0$ such that the linearized operator $L_{m, k}$ has at least one eigenvalue outside the imaginary axis. The strategy is then to perform a homotopy between the vorticity profile $W_{0}:=W$ and a reference profile $W_{1}$ for which we know a priori that the corresponding linearized operator has no eigenvalue with nonzero real part. Since eigenvalues outside the imaginary axis depend continuously on the vorticity profile, in an appropriate topology, this implies in our situation that all eigenvalues necessarily merge into the essential spectrum as the homotopy parameter varies from zero to one. We eventually reach a contradiction by showing that such a merger is impossible. This is achieved by a careful asymptotic analysis of the solutions of the complex ODE (3.2) in the limit where the real part of the eigenvalue $s=m(a-i b)$ vanishes. Our approach combines the results of Section 3.4 on critical layers, the integral identities obtain by Howard's method in Section 3.3 , and new ingredients which rely on the monotonicity assumption $\mathrm{H} 2$.

Since we have to consider various vorticity profiles in the course the proof, the linearized operator (2.1) will sometimes be denoted by $L_{m, k}^{W}$ instead of $L_{m, k}$, to avoid any ambiguity. The following continuity property plays an essential role in our argument.

Lemma 4.1. The (linear) mapping $W \mapsto L_{m, k}^{W}$ is continuous from $\mathcal{C}_{b}^{1}\left(\overline{\mathbb{R}}_{+}\right)$into $\mathcal{L}\left(X_{m, k}\right)$. 
Proof. As can be seen from definitions (2.1)-(2.3), the linearized operator $L_{m, k}^{W}$ has variable coefficients depending (linearly) on the functions $\Omega, r \Omega^{\prime}, W$, and $W^{\prime}$. Now, we have the estimate

$$
\|\Omega\|_{L^{\infty}\left(\mathbb{R}_{+}\right)}+\left\|r \Omega^{\prime}\right\|_{L^{\infty}\left(\mathbb{R}_{+}\right)} \leq C\|W\|_{L^{\infty}\left(\mathbb{R}_{+}\right)},
$$

which follows from the representation formula (1.16) and the identity $r \Omega^{\prime}=W-2 \Omega$. Thus all coefficients of $L_{m, k}^{W}$ are $L^{\infty}$ functions that depend continuously on $W$ in the topology of $\mathcal{C}_{b}^{1}\left(\overline{\mathbb{R}}_{+}\right)$, and since the Biot-Savart map $\omega \mapsto u$ is bounded in $X_{m, k}$ by Proposition 6.1 below, we obtain the desired result.

\subsection{Reduction to a real analytic vorticity profile}

We now present the contradiction argument in detail. We fix $m \geq 1, k>0$, and we assume that there exists a vorticity profile $W_{0} \in \mathscr{W}$ such that the associated linear operator $L_{m, k}^{W_{0}} \in \mathcal{L}\left(X_{m, k}\right)$ has at least one (isolated) eigenvalue outside the imaginary axis. Our goal is to prove that this is impossible, which is exactly the conclusion of Theorem [1.3.

In a first step, we show that one can assume without loss of generality that the profile $W_{0} \in \mathscr{W}$ is real analytic on $\overline{\mathbb{R}}_{+}$. By this we mean more precisely that $W_{0}$ is the restriction to $\overline{\mathbb{R}}_{+}$of a real analytic even function defined on the whole real line. Indeed, we know from Proposition 2.1 that, for any $W \in \mathscr{W}$, the spectrum of $L_{m, k}^{W}$ outside the imaginary axis consists of isolated eigenvalues with finite multiplicity, which are in fact simple as asserted by Lemma 3.1. Invoking Lemma 4.1 and classical perturbation theory [15, IV-§3.5], we observe that these (hypothetical) eigenvalues depend continuously on the vorticity profile $W$ in the topology of $\mathcal{C}_{b}^{1}\left(\overline{\mathbb{R}}_{+}\right)$. In particular, if $W$ is close enough to $W_{0}$ in that topology, we are sure that the operator $L_{m, k}^{W}$ has at least one eigenvalue with nonzero real part.

We next invoke a density result that will be established in Section 6.4 below.

Lemma 4.2. The subset $\mathscr{W}^{\omega}$ of $\mathscr{W}$ consisting of vorticity profiles which are also real analytic on $\overline{\mathbb{R}}_{+}$is dense in $\mathscr{W}$ for the topology of $\mathcal{C}_{b}^{1}\left(\overline{\mathbb{R}}_{+}\right)$.

The proof of Lemma 4.2 is not straightforward because the definition of the class $\mathscr{W}$ involves the quantity $J$, introduced in (1.20), which depends in a nonlinear way on the vorticity profile $W$. Thus, given $W \in \mathscr{W}$, we cannot construct an approximation $W_{\epsilon} \in \mathscr{W}^{\omega}$ just by taking the convolution of $W$ with a real analytic mollifier. To avoid this difficulty, we prove in Section 6.4 that all quantities $\Omega, W, \Phi$ are entirely determined by the auxiliary function $J$, and we even provide explicit reconstruction formulas. Then, at the level of $J$, we use a nonlinear approximation scheme of the form

$$
\frac{1}{\sqrt{1+J_{\epsilon}}}=G_{\epsilon} * \frac{1}{\sqrt{1+J}}, \quad \epsilon>0
$$

where $G_{\epsilon}$ denotes the heat kernel on the half-line $\mathbb{R}_{+}$with Dirichlet boundary condition at $r=0$. This provides an approximation procedure within the class $\mathscr{W}$ which allows us to prove Lemma 4.2, see Section 6.4 for details.

Taking advantage of Lemma 4.2 we assume from now on that the initial vorticity profile $W_{0}$ in our contradiction argument is real analytic, namely $W_{0} \in \mathscr{W}^{\omega}$.

\subsection{Construction of the homotopy}

In the particular example of the Kaufmann-Scully vortex (1.8), the function (1.20) reduces to $J(r)=1+1 / r^{2} \geq 1$. By a simple rescaling we deduce that, for the vorticity profile $W_{1} \in \mathscr{W}^{\omega}$ 
defined by

$$
W_{1}(r)=\frac{2}{\left(1+4 k^{2} r^{2} / m^{2}\right)^{2}}=\frac{2 m^{4}}{\left(m^{2}+4 k^{2} r^{2}\right)^{2}}, \quad r \geq 0,
$$

the stability condition (3.17) is satisfied, so that the linear operator $L_{m, k}^{W_{1}}$ has no eigenvalue outside the imaginary axis as a consequence of Proposition 3.4. To interpolate in the class $\mathscr{W}$ between the initial profile $W_{0} \in \mathscr{W}^{\omega}$ and the reference profile (4.1), we use the following result, whose proof is also postponed to Section 6.4

Lemma 4.3. If $W_{0}, W_{1} \in \mathscr{W}$, there exists a Lipschitz function $\mathcal{H}:[0,1] \rightarrow \mathcal{C}_{b}^{1}\left(\overline{\mathbb{R}}_{+}\right)$such that $\mathcal{H}(0)=W_{0}, \mathcal{H}(1)=W_{1}$, and $W_{t}:=\mathcal{H}(t) \in \mathscr{W}$ for any $t \in[0,1]$. Moreover, if $W_{0}, W_{1} \in \mathscr{W}^{\omega}$, then $W_{t} \in \mathscr{W}^{\omega}$ for all $t \in[0,1]$. In that case, if $W_{1}^{\prime \prime}(0)<0$, then $W_{t}^{\prime \prime}(0)<0$ for all $t \in(0,1]$.

Since the class $\mathscr{W}$ is not convex, the linear interpolation $H(t)=(1-t) W_{0}+t W_{1}$ is not appropriate here. Instead, we use again the fact that a vorticity profile $W \in \mathscr{W}$ is entirely determined by the auxiliary function (1.20), and at the level of $J$ we define the homotopy $\mathcal{H}$ by the following nonlinear interpolation procedure

$$
\frac{1}{\sqrt{1+J_{t}}}=\frac{1-t}{\sqrt{1+J_{0}}}+\frac{t}{\sqrt{1+J_{1}}}, \quad \forall t \in[0,1] .
$$

If $J_{0}$ and $J_{1}$ are real analytic, so is $J_{t}$ for all $t \in[0,1]$, and it follows that $W_{t} \in \mathscr{W}^{\omega}$ for all $t \in[0,1]$. We refer to Section 6.4 for details.

\subsection{The bifurcation point}

For any $t \in[0,1]$, we denote by $W_{t} \in \mathscr{W}^{\omega}$ the vorticity profile obtained from Lemma 4.3, where $W_{0} \in \mathscr{W}^{\omega}$ is the initial vorticity defined in Section 4.1 and $W_{1}$ is given by (4.1). We also introduce the associated angular velocity

$$
\Omega_{t}(r)=\frac{1}{r^{2}} \int_{0}^{r} W_{t}(s) s \mathrm{~d} s, \quad r>0
$$

and we define $\Phi_{t}=2 \Omega_{t} W_{t}$ and $J_{t}=\Phi_{t} /\left(\Omega_{t}^{\prime}\right)^{2}$ as in (1.19), (1.20). We consider the family of linear operators $L_{m, k}^{W_{t}}$, indexed by the homotopy parameter $t \in[0,1]$, which is uniformly bounded in $\mathcal{L}\left(X_{m, k}\right)$ by Lemma 4.1, For each $t \in[0,1]$, it follows from Proposition 2.1 and Lemma 3.1 that the spectrum of $L_{m, k}^{W_{t}}$ outside the imaginary axis consists of simple isolated eigenvalues. If $s=m(a-i b)$ is such an eigenvalue, we know from Proposition 3.4 that $0<b<1$, and by uniform boundedness there exists a constant $M>0$ (independent of $t$ ) such that $0<|a| \leq M$.

As the homotopy parameter $t$ varies, the isolated eigenvalues of $L_{m, k}^{W_{t}}$ move continuously in the complex plane, as described e.g. in [15, IV-§3.5], and we chose our reference profile $W_{1}$ so that the associated linearized operator has no eigenvalue with nonzero real part. This implies that, when $t$ increases from zero to one, all isolated eigenvalues of $L_{m, k}^{W_{t}}$ eventually merge into the essential spectrum on the imaginary axis. In particular, we can define the bifurcation point

$$
t_{*}=\inf \left\{t \in(0,1] \mid \sigma\left(L_{m, k}^{W_{\tau}}\right) \subset i \mathbb{R} \text { for all } \tau \in[t, 1]\right\} .
$$

Our assumption on $W_{0}$ and the continuity of the eigenvalues imply that $t_{*}>0$ and $\sigma\left(L_{m, k}^{W_{t_{*}}}\right) \subset i \mathbb{R}$. Moreover, there exist an increasing sequence $t_{n} \rightarrow t_{*}$ and a sequence of isolated eigenvalues $s_{n}=m\left(a_{n}-i b_{n}\right)$ of $L_{m, k}^{W_{t_{n}}}$ such that $a_{n} \neq 0,0<b_{n}<1$, and

$$
a_{n}+i b_{n} \rightarrow i \bar{b}, \quad \text { as } \quad n \rightarrow \infty
$$


for some $\bar{b} \in[0,1]$. In view of the second identity in (2.6), we can assume without loss of generality that $a_{n}>0$ for all $n \in \mathbb{N}$. Associated with $\bar{b}$, we also introduce the critical radius

$$
\bar{r}= \begin{cases}\Omega_{t_{*}}^{-1}(\bar{b}) & \text { if } \bar{b}>0 \\ +\infty & \text { if } \bar{b}=0\end{cases}
$$

As $W_{t_{*}} \in \mathscr{W}^{\omega}$ by construction, we recall that $\Omega_{t_{*}}: \overline{\mathbb{R}}_{+} \rightarrow \mathbb{R}_{+}$is real analytic, strictly decreasing on $\mathbb{R}_{+}$, and satisfies $\Omega_{t_{*}}(0)=1$ and $\Omega_{t_{*}}(r) \rightarrow 0$ as $r \rightarrow \infty$, so that $\bar{r} \in[0, \infty]$ is well defined, see Fig. 1.

In the sequel, for notational simplicity, we write $W$ instead of $W_{t_{*}}$ and $W_{n}$ instead of $W_{t_{n}}$. Note in particular that, after this redefinition, the symbol $W$ no longer refers to the vorticity profile that appears in the statement of Theorem [1.3]! Similarly, we denote

$$
\Omega=\Omega_{t_{*}}, \Phi=\Phi_{t_{*}}, J=J_{t_{*}}, \quad \text { and } \quad \Omega_{n}=\Omega_{t_{n}}, \Phi_{n}=\Phi_{t_{n}}, J_{n}=J_{t_{n}} .
$$

Finally, we also set $L_{m, k}=L_{m, k}^{W}$ and $L_{m, k}^{n}=L_{m, k}^{W_{n}}$. We observe that $W_{n} \rightarrow W$ in $\mathcal{C}_{b}^{1}\left(\overline{\mathbb{R}}_{+}\right)$as $n \rightarrow \infty$, due to the continuity properties of the homotopy defined in Lemma 4.3 .

As is recalled at the beginning of Section 3 , for each $n \in \mathbb{N}$ we may associate to the eigenvalue $s_{n}=m\left(a_{n}-i b_{n}\right)$ of $L_{m, k}^{n}$ a nontrivial solution $u_{n} \in H^{1}\left(\mathbb{R}_{+}, r \mathrm{~d} r\right) \cap H_{\mathrm{loc}}^{2}\left(\mathbb{R}_{+}\right)$of the complex differential equation

$$
-\partial_{r}\left(\mathcal{A}(r) \partial_{r}^{*} u_{n}\right)+\left[1-\frac{k^{2}}{m^{2}} \frac{\mathcal{A}(r) \Phi_{n}(r)}{\gamma_{n}(r)^{2}}+\frac{r}{\gamma_{n}(r)} \partial_{r}\left(\frac{W_{n}(r)}{m^{2}+k^{2} r^{2}}\right)\right] u_{n}=0,
$$

where $\gamma_{n}(r)=\Omega_{n}(r)-b_{n}-i a_{n}$. As $W_{n} \in \mathscr{W}^{\omega}$, it is clear that $u_{n}$ is in fact real analytic for all $n \in \mathbb{N}$. According to Lemma 3.1 there exist nonzero complex numbers $\alpha_{n}, \beta_{n}$ such that

$$
\alpha_{n}=\lim _{r \rightarrow 0_{+}} r^{-m+1} u_{n}(r), \quad \text { and } \quad \beta_{n}=\lim _{r \rightarrow \infty} r^{1 / 2} \exp (k r) u_{n}(r) .
$$

In what follows, we often normalize $u_{n}$ so that $\beta_{n}=1$ for all values of $n$.

As $n \rightarrow \infty$, the ODE (4.5) becomes singular at the point $r=\bar{r}$, because $\gamma_{n}(\bar{r}) \rightarrow \Omega(\bar{r})-\bar{b}=0$ in view of (4.3) and (4.4). As is explained in Section 3.2, the nature of the critical layer near $r=\bar{r}$ depends upon whether the quantity $J(\bar{r})$ is larger or smaller than $m^{2} /\left(4 k^{2}\right)$. This motivates the following definition:

$$
r_{*}= \begin{cases}J^{-1}\left(\frac{m^{2}}{4 k^{2}}\right) & \text { if inf } J<\frac{m^{2}}{4 k^{2}} \\ +\infty & \text { otherwise }\end{cases}
$$

Note that $J:(0, \infty) \rightarrow \mathbb{R}_{+}$is strictly decreasing by assumption $\mathrm{H} 2$, so that $r_{*}$ is uniquely defined. Moreover,

$$
r_{*}>0, \quad J(r)>\frac{m^{2}}{4 k^{2}} \text { for } r<r_{*}, \quad \text { and } J(r)<\frac{m^{2}}{4 k^{2}} \text { for } r>r_{*} .
$$

Also, since $t_{*}>0$ we deduce from (4.2) and from our choice (4.1) of $W_{1}$ that

$$
J(\infty)>0
$$

In the rest of the proof of Theorem 1.3, to reach the desired contradiction, we consider various cases according to whether the critical radius $\bar{r}$ is larger, smaller or equal to $0, r_{*}$, or $+\infty$. 


\subsection{The situation $0<\bar{r}<r_{*}$ is excluded}

In this case, a contradiction is obtained from identity (3.16), or rather from its equivalent for the solutions of (4.5) where the vorticity profile $W_{n}$ and the spectral parameter $s_{n}=m\left(a_{n}-i b_{n}\right)$ depend on $n$. In terms of the weighted function

$$
v_{n}(r)=\frac{u_{n}(r)}{\left(b_{n}+i a_{n}-\Omega_{n}(r)\right)^{1 / 2}}=\frac{-i u_{n}(r)}{\gamma_{n}(r)^{1 / 2}}, \quad r>0,
$$

the identity becomes, after dividing by $a_{n} \neq 0$ :

$$
\int_{0}^{\infty}\left\{\mathcal{A}(r)\left|\partial_{r}^{*} v_{n}\right|^{2}+\left|v_{n}\right|^{2}+\frac{\mathcal{A}(r) \Omega_{n}^{\prime}(r)^{2}}{a_{n}^{2}+\left(\Omega_{n}(r)-b_{n}\right)^{2}}\left(\frac{k^{2}}{m^{2}} J_{n}(r)-\frac{1}{4}\right)\left|v_{n}\right|^{2}\right\} r \mathrm{~d} r=0 .
$$

We choose here to normalize the solutions $u_{n}$ of (4.5) so that $\beta_{n}=1$ in (4.6) for all $n \in \mathbb{N}$. This implies, in view of the analysis in Sections 3.1 and 3.4 and of the definitions in Section 4.3, that $u_{n}(r) \rightarrow \psi_{\infty}(r)$ and $u_{n}^{\prime}(r) \rightarrow \psi_{\infty}^{\prime}(r)$ locally uniformly on $(\bar{r}, \infty]$ as $n \rightarrow \infty$, where $\psi_{\infty}$ is the solution of the limiting equation (3.18) introduced in Lemma 3.7. Moreover, for any $\varepsilon>0$, the sequence $\left(u_{n}\right)$ is uniformly bounded in $H^{1}([\bar{r}+\varepsilon, \infty), r \mathrm{~d} r)$, and so is the sequence $\left(v_{n}\right)$ since $\left|\gamma_{n}(r)\right|$ is bounded away from zero when $r \geq \bar{r}+\varepsilon$. This uniform $H^{1}$ bound means that the restriction of the integral in (4.11) to the interval $[\bar{r}+\varepsilon, \infty)$ is uniformly bounded for all $n \in \mathbb{N}$. As the integral over $(0,+\infty)$ is equal to zero, we deduce that the integral over $(0, \bar{r}+\varepsilon)$ is also uniformly bounded, namely

$$
\sup _{n \in \mathbb{N}} \int_{0}^{\bar{r}+\varepsilon}\left\{\mathcal{A}(r)\left|\partial_{r}^{*} v_{n}\right|^{2}+\left|v_{n}\right|^{2}+\frac{\mathcal{A}(r) \Omega_{n}^{\prime}(r)^{2}}{a_{n}^{2}+\left(\Omega_{n}(r)-b_{n}\right)^{2}}\left(\frac{k^{2}}{m^{2}} J_{n}(r)-\frac{1}{4}\right)\left|v_{n}\right|^{2}\right\} r \mathrm{~d} r<\infty .
$$

Now comes into play the assumption that $\bar{r}<r_{*}$. If we choose $\varepsilon>0$ small enough so that $\bar{r}+\varepsilon<r_{*}$, we observe that, due to the definition of $r_{*}$ in (4.7), the integrand in (4.12) is nonnegative when $n$ is sufficiently large. Moreover, for all $r>\bar{r}$, we know from (4.10) that $v_{n}(r) \rightarrow \psi_{\infty}(r)(\bar{b}-\Omega(r))^{-1 / 2}$ as $n \rightarrow \infty$. So restricting the integral to the interval $(\bar{r}, \bar{r}+\varepsilon)$ and invoking Fatou's lemma, we deduce from (4.12) that

$$
\int_{\bar{r}}^{r_{*}} \frac{\mathcal{A}(r) \Omega^{\prime}(r)^{2}}{(\bar{b}-\Omega(r))^{3}}\left(\frac{k^{2}}{m^{2}} J(r)-\frac{1}{4}\right)\left|\psi_{\infty}(r)\right|^{2} r \mathrm{~d} r<\infty .
$$

The inequality $\bar{r}<r_{*}$ also means that the roots of the indicial equation (3.19) are complex conjugate so that, according to what we called Case 2 in Section 3.4, we have the decomposition $\psi_{\infty}(r)=\alpha_{-}^{\infty} \phi_{-}(r)+\alpha_{+}^{\infty} \phi_{+}(r)$ for $r>\bar{r}$, where $\phi_{ \pm}$are given by (3.21). From these expressions, it is easy to deduce that (4.13) cannot hold if $\psi_{\infty}$ is replaced by either $\phi_{+}$or $\phi_{-}$, because the integrand is positive and behaves like $(r-\bar{r})^{-2}$ in a neighborhood of $\bar{r}$. In the general case where both coefficients $\alpha_{ \pm}^{\infty}$ are nonzero, there may be cancellations between the contributions of $\phi_{+}$and $\phi_{-}$, but due to the logarithmic phases in the expressions (3.21) of $\phi_{ \pm}$the function $\psi_{\infty}$ cannot vanish on large sets. More precisely, given $\alpha_{ \pm}^{\infty} \in \mathbb{C}$, there exist $\epsilon>0$ and $0<\rho_{0}<r_{*}-\bar{r}$ such that, for any $\rho \in\left(0, \rho_{0}\right)$,

$$
\frac{1}{\rho} \text { meas }\left(\left\{r \in(\bar{r}, \bar{r}+\rho) \text { s.t. }\left|\psi_{\infty}(r)\right|^{2} \geq \epsilon|r-\bar{r}|\right\}\right) \geq \frac{1}{2},
$$

and the same argument as above shows that (4.13) is impossible. 


\subsection{The situation $\bar{r}=0$ is excluded}

This case is treated by the same argument as in the previous section up to and including inequality (4.13). The only difference at that level is the asymptotic behavior of the functions $\phi_{ \pm}(r)$ as $r \rightarrow \bar{r}$, because $\bar{r}=0$ is now an irregular singular point of the ODE (3.18). According to (3.9), we have the expansion

$$
\phi_{ \pm}(r)=\frac{1}{\sqrt{r}} \exp \left(\frac{ \pm 16 i k}{m W^{\prime \prime}(0) r}\right)(1+\mathcal{O}(r)), \quad \text { as } r \rightarrow 0_{+} .
$$

The contradiction then follows exactly as in Section 4.4, the integrand of (4.13) being even more singular here since it behaves like $r^{-4}$ in a neighborhood of 0 .

\subsection{The situation $r_{*}<\bar{r}<\infty$ is excluded}

In that case, we cannot get a contradiction from identity (4.11), because the various terms in the integrand now have different signs in a neighborhood of the singular point $\bar{r}$. Instead, our argument relies on a detailed analysis of the solutions of (4.5) near $\bar{r}$, and on monotonicity properties that follow from assumption $\mathrm{H} 2$.

As in the previous section, we normalize the solutions $u_{n}$ of (4.5) so that $\beta_{n}=1$ in (4.6) for all $n \in \mathbb{N}$. In particular, for any $r>\bar{r}$, we know that $u_{n}(r) \rightarrow \psi_{\infty}(r)$ and $u_{n}^{\prime}(r) \rightarrow \psi_{\infty}^{\prime}(r)$ as $n \rightarrow \infty$, where $\psi_{\infty}$ is as in Lemma 3.7. Applying Lemma 3.5, whose assumptions are satisfied by construction of the homotopy argument, we deduce that

$$
u_{n}(r) \rightarrow \alpha_{-}^{\infty} \phi_{-}(r)+\alpha_{+}^{\infty} \phi_{+}(r), \quad \text { and } \quad u_{n}^{\prime}(r) \rightarrow \alpha_{-}^{\infty} \phi_{-}^{\prime}(r)+\alpha_{+}^{\infty} \phi_{+}^{\prime}(r),
$$

for all $r \in(0, \infty) \backslash \bar{r}$, where $\alpha_{ \pm}^{\infty} \in \mathbb{C}$ and $\phi_{ \pm}$are the solutions of (3.18) given by (3.20). Note that the roots $d_{ \pm}$of the indicial equation (3.19) are now real and distinct, so that we are in the situation referred to as Case 1 in Section 3.4. The convergence (4.15) for some $r<\bar{r}$ implies, in view of the results in Section 3.1 concerning the solutions of (3.2) near the origin, that the normalizing constants $\alpha_{n}$ in (4.6) converge to some limit $\alpha_{*} \in \mathbb{C}$ as $n \rightarrow \infty$. We deduce that

$$
\alpha_{-}^{\infty} \phi_{-}(r)+\alpha_{+}^{\infty} \phi_{+}(r)= \begin{cases}\alpha_{*} \psi_{0}(r) & \text { if } r \in(0, \bar{r}) \\ \psi_{\infty}(r) & \text { if } r \in(\bar{r}, \infty)\end{cases}
$$

Now, the functions $\psi_{\infty}, \phi_{-}, \phi_{+}$are all real-valued on $(\bar{r}, \infty)$, and we know from (3.20) that $\phi_{ \pm}(r) \approx(r-\bar{r})^{d_{ \pm}}$as $r \rightarrow \bar{r}_{+}$, where $0<d_{-}<1 / 2<d_{+}<1$. These observations imply that both coefficients $\alpha_{-}^{\infty}$ and $\alpha_{+}^{\infty}$ are necessarily real. On the other hand, we deduce from (4.16) that the complex function $\alpha_{-}^{\infty} \phi_{-}+\alpha_{+}^{\infty} \phi_{+}$must have a constant phase (modulo $\pi$ ) on the interval $(0, \bar{r})$, as it is equal to the product of the real function $\psi_{0}$ by the complex constant $\alpha_{*}$. This, however, is impossible if both coefficients $\alpha_{ \pm}^{\infty}$ are nonzero, because by (3.20) the complex functions $\phi_{ \pm}$ have different phases when $r<\bar{r}$ and vanish at different rates as $r \rightarrow \bar{r}_{-}$. More precisely, since $\phi_{+}(r) / \phi_{-}(r) \rightarrow 0$ as $r \rightarrow \bar{r}_{-}$, it follows from (4.16), (3.20) that

$$
\alpha_{-}^{\infty}=\alpha_{*} e^{-i \pi d_{-}} \lambda, \quad \text { where } \quad \lambda=\lim _{r \rightarrow \bar{r}_{-}} \frac{\psi_{0}(r)}{|\bar{b}-\Omega(r)|^{d_{-}}} \in \mathbb{R} .
$$

If $\alpha_{-}^{\infty} \neq 0$, then $\lambda \neq 0$ and $\alpha_{*}=\alpha_{-}^{\infty} \lambda^{-1} e^{i \pi d_{-}}$, so using (4.16) and (3.20) again we obtain

$$
0=\operatorname{Im}\left(\alpha_{+}^{\infty} e^{-i \pi d_{-}} \phi_{+}(r)\right)=\alpha_{+}^{\infty} \sin \left(\pi\left(d_{+}-d_{-}\right)\right) V_{+}(r), \quad 0<r<\bar{r} .
$$


As $V_{+}(r) \neq 0$ for $r$ sufficiently close to $\bar{r}$, we conclude that $\alpha_{+}^{\infty} \sin \left(\pi\left(d_{+}-d_{-}\right)\right)=0$, and this implies that $\alpha_{+}^{\infty}=0$ since $0<d_{+}-d_{-}<1$. Therefore, we must have $\alpha_{-}^{\infty} \alpha_{+}^{\infty}=0$.

In the rest of this section, using totally different arguments which rely on assumption $\mathrm{H} 2$, we show that necessarily $\alpha_{-}^{\infty} \alpha_{+}^{\infty} \neq 0$ in (4.16), and this will give the desired contradiction. To that purpose, we introduce the auxiliary functions

$$
U_{ \pm}(r)=(\bar{b}-\Omega(r))^{d_{ \pm}}, \quad r>\bar{r},
$$

and we denote by $\mathscr{L}=-\partial_{r} \mathcal{A}(r) \partial_{r}^{*}+\mathcal{B}(r)$ the linear operator in (3.18). We claim that:

Lemma 4.4 (Upper solutions). There exists $\gamma>0$ such that $\mathscr{L}\left(U_{ \pm}\right) \geq \gamma U_{ \pm}^{\prime}>0$ on $(\bar{r}, \infty)$.

Proof. For notational simplicity we write $U$ instead of $U_{ \pm}, d$ instead of $d_{ \pm}$, and $b$ instead of $\bar{b}$. Computing $\mathscr{L}(U)$ when $U=(b-\Omega)^{d}$, we obtain after elementary rearrangements of terms

$$
\mathscr{L}(U)=(b-\Omega)^{d-2}\left(T_{1}+T_{2}+T_{3}\right),
$$

where

$$
T_{1}=-\mathcal{A} \Omega^{\prime 2}\left(d(d-1)+\frac{k^{2}}{m^{2}} J\right), \quad T_{2}=\left(1-\left(\frac{\mathcal{A}}{r}\right)^{\prime}\right)(b-\Omega)^{2},
$$

and

$$
T_{3}=\mathcal{A}(b-\Omega)\left(d \Omega^{\prime \prime}+d\left(\frac{\mathcal{A}^{\prime}}{\mathcal{A}}+\frac{1}{r}\right) \Omega^{\prime}-\frac{r}{\mathcal{A}} \partial_{r}\left(\frac{W}{m^{2}+k^{2} r^{2}}\right)\right) .
$$

Since $d$ is a solution of the indicial equation (3.19), we may rewrite

$$
T_{1}(r)=\mathcal{A}(r){\Omega^{\prime}}^{2}(r) \frac{k^{2}}{m^{2}}(J(\bar{r})-J(r)),
$$

and our assumption $\mathrm{H} 2$ on $J$ implies that $T_{1}>0$ on $(\bar{r}, \infty)$. Next, using the definition of $\mathcal{A}$ and the fact that $m^{2} \geq 1$, we observe that

$$
1-\left(\frac{\mathcal{A}}{r}\right)^{\prime}=1-\frac{m^{2}-k^{2} r^{2}}{\left(m^{2}+k^{2} r^{2}\right)^{2}} \geq 0,
$$

so that $T_{2} \geq 0$ on $(0, \infty)$. As for $T_{3}$, we expand

$$
\frac{\mathcal{A}^{\prime}}{\mathcal{A}}+\frac{1}{r}=\frac{3}{r}-\frac{2 k^{2} r}{m^{2}+k^{2} r^{2}}, \quad \frac{r}{\mathcal{A}} \partial_{r}\left(\frac{W}{m^{2}+k^{2} r^{2}}\right)=\frac{W^{\prime}}{r}-\frac{2 k^{2} W}{m^{2}+k^{2} r^{2}},
$$

and we use the identities $W=r \Omega^{\prime}+2 \Omega$ and $W^{\prime}=r \Omega^{\prime \prime}+3 \Omega^{\prime}$ to derive the alternative expression

$$
T_{3}=\mathcal{A}(b-\Omega)\left((d-1) \frac{W^{\prime}}{r}+\frac{2 k^{2}}{m^{2}+k^{2} r^{2}}\left(W-d r \Omega^{\prime}\right)\right) .
$$

Since $0<d<1$, and since $W>0, W^{\prime}<0$ and $\Omega^{\prime}<0$ by assumption H1, we deduce that $T_{3}$ as well is positive on $(\bar{r}, \infty)$. Altogether, we have shown that $\mathscr{L}(U)>0$ on $(\bar{r}, \infty)$.

To conclude the proof, we fix $r_{0}>\bar{r}$ large enough so that $\left|(\mathcal{A} / r)^{\prime}\right| \leq 1 / 2$ for $r \geq r_{0}$. In that region, we have $\mathscr{L}(U) \geq(b-\Omega)^{d-2} T_{2} \geq(b-\Omega)^{d} / 2 \geq \gamma_{0} U^{\prime}$ if $\gamma_{0}>0$ is small enough. On the other hand, using (4.18) and the fact that $J^{\prime}(\bar{r})<0, \Omega(\bar{r})=b$, and $\Omega^{\prime}(\bar{r})<0$, we can find $\gamma_{1}>0$ small enough so that

$$
T_{1}(r)=\frac{J(\bar{r})-J(r)}{b-\Omega(r)} \mathcal{A}(r){\Omega^{\prime 2}}^{2}(r) \frac{k^{2}}{m^{2}}(b-\Omega(r)) \geq \gamma_{1} d(b-\Omega(r))\left|\Omega^{\prime}(r)\right|,
$$

for all $r$ in the compact interval $\left[\bar{r}, r_{0}\right]$. This implies that $\mathscr{L}(U) \geq \gamma_{1} U^{\prime}$ on $\left[\bar{r}, r_{0}\right]$, and taking $\gamma=\min \left(\gamma_{0}, \gamma_{1}\right)$ we obtained the desired conclusion. 
Corollary 4.5. The solutions $\phi_{ \pm}(r)$ of (3.18) given by (3.20) are unbounded as $r \rightarrow \infty$.

Proof. Assume that $\varphi$ is a solution of $\mathscr{L}(\varphi)=0$ on $(\bar{r}, \infty)$, which is decomposed as $\varphi=U V$ where $U$ is one of the functions $U_{ \pm}$defined in (4.17). The equation satisfied by $V$ is

$$
0=\mathscr{L}(U V)=-\mathcal{A} U V^{\prime \prime}-\left(2 \mathcal{A} U^{\prime}+\left(\mathcal{A}^{\prime}+\mathcal{A} / r\right) U\right) V^{\prime}+\mathscr{L}(U) V, \quad r \in(\bar{r}, \infty) .
$$

We interpret the right-hand side of (4.19) as the action on the function $V$ of a second order differential operator $\mathscr{L}_{U}$ whose coefficients depend on $U$. Since $\mathcal{A} U$ is positive on $(\bar{r}, \infty)$ by construction, and $\mathscr{L}(U)$ is positive on $(\bar{r}, \infty)$ by Lemma 4.4, we observe that the maximum principle holds for the operator $\mathscr{L}_{U}$. As a consequence, the function $V$ which satisfies $\mathscr{L}_{U}(V)=0$ cannot have a positive maximum nor a negative minimum on the interval $(\bar{r}, \infty)$.

We first choose $\varphi=\varphi_{+}, U=U_{-}$, and we claim that $\varphi_{+}$is unbounded on $(\bar{r}, \infty)$. Indeed, in the opposite case, the function $V(r)=\phi_{+}(r) / U_{-}(r)$ would tend to zero both as $r \rightarrow \bar{r}_{+}$and as $r \rightarrow \infty$, so that $V \equiv 0$ by the maximum principle, which is clearly absurd. As a second application, we take $\varphi=\varphi_{-}, U=U_{-}$, and we claim again that $\varphi_{-}$is unbounded on $(\bar{r}, \infty)$. If not, by the maximum principle the function $V(r)=\phi_{-}(r) / U_{-}(r)$ would be nonincreasing on $(\bar{r}, \infty)$ with $V(r) \rightarrow 1$ as $r \rightarrow \bar{r}_{+}$and $V(r) \rightarrow 0$ as $r \rightarrow \infty$. Note that $V$ coincides with the function $V_{-}$in (3.20) and is therefore analytic up to the singular point $\bar{r}$. Thus, using equation (4.19) and Lemma 4.4, we can compute

$$
V^{\prime}(\bar{r})=\lim _{r \rightarrow \bar{r}_{+}} V^{\prime}(r)=\lim _{r \rightarrow \bar{r}_{+}} \frac{\mathscr{L}(U) V-\mathcal{A} U V^{\prime \prime}}{2 \mathcal{A} U^{\prime}+\left(\mathcal{A}^{\prime}+\mathcal{A} / r\right) U}=\lim _{r \rightarrow \bar{r}_{+}} \frac{\mathscr{L}(U)}{2 \mathcal{A} U^{\prime}} \geq \frac{\gamma}{2 \mathcal{A}(\bar{r})}>0,
$$

and this contradicts the claim that $V$ is nonincreasing. The proof is complete.

As $\psi_{\infty}$ is bounded on the interval $(\bar{r}, \infty)$ whereas both $\phi_{+}, \phi_{-}$are unbounded by Corollary 4.5, the relation $\psi_{\infty}=\alpha_{-}^{\infty} \phi_{-}+\alpha_{+}^{\infty} \phi_{+}$can hold only if both coefficients $\alpha_{-}^{\infty}, \alpha_{+}^{\infty}$ are nonzero. This gives the desired contradiction in the case where $r_{*}<\bar{r}<\infty$.

\subsection{The situation $\bar{r}=r_{*}<\infty$ is excluded}

We proceed here as in Section 4.6. the only essential difference being that the exponents $d_{-}=$ $d_{+}=1 / 2$ are no longer distinct. We are thus in the situation referred to as Case 3 in Section 3.4 , where the solutions $\phi_{ \pm}$are given by the expressions (3.22). Applying Corollary 4.5, we obtain as above that $\phi_{+}$is unbounded on the interval $(\bar{r}, \infty)$, but the argument does not apply to the second solution $\phi_{-}$which contains a logarithmic correction, see (3.22). Nevertheless, we deduce that the coefficient $\alpha_{-}^{\infty}$ in the representation (4.16) is necessarily nonzero, and this turns out to be enough to obtain the desired contradiction. Indeed, due to the logarithmic term, it is easy to verify that, if $\theta(r)=\arg \left(\alpha_{-}^{\infty} \phi_{-}(r)+\alpha_{+}^{\infty} \phi_{+}(r)\right)$, then $\tan (\theta(r)) \sim-\pi^{-1} \log (\bar{r}-r)$ as $r \rightarrow \bar{r}$, which shows that the left-hand side of (4.16) cannot have a constant phase for $r \in(0, \bar{r})$.

\subsection{The situation $r_{*}<\bar{r}=\infty$ is excluded}

We next consider the case where $\bar{b}=0$ in (4.3), so that $\bar{r}=+\infty$ according to (4.4). In that situation, the "critical layer" occurs at very large values of $r$, in a region where the eigenvalue equation (4.5) is already in some asymptotic regime. Here we cannot use the same arguments as in section 4.6 to obtain a contradiction, because the location of the critical layer changes as $n$ is increased. However, it is possible to obtain an accurate representation of the solution of (4.5) that decays to zero as $r \rightarrow \infty$, by comparing it with the explicit solution of a model problem, 
(4.25) below, which can be expressed in terms of modified Bessel functions. This approximation turns out to be sufficient to derive a contradiction when combined with the identity (3.11).

Our starting point is the equation (3.5) for $w_{n}(r)=r^{1 / 2} u_{n}(r)$, which reads

$$
w_{n}^{\prime \prime}(r)+\frac{\mathcal{A}^{\prime}(r)}{\mathcal{A}(r)} w_{n}^{\prime}(r)-\mathcal{D}_{n}(r) w_{n}(r)=0, \quad r>0,
$$

where, in view of (3.3),

$$
\mathcal{D}_{n}(r)=k^{2}+\frac{m^{2}+\frac{3}{4}}{r^{2}}-\frac{1}{2 r} \frac{\mathcal{A}^{\prime}(r)}{\mathcal{A}(r)}-\frac{k^{2}}{m^{2}} \frac{\Phi_{n}(r)}{\gamma_{n}(r)^{2}}+\frac{r}{\mathcal{A}(r) \gamma_{n}(r)} \partial_{r}\left(\frac{W_{n}(r)}{m^{2}+k^{2} r^{2}}\right) .
$$

We recall that $\gamma_{n}(r)=\Omega_{n}(r)-b_{n}-i a_{n}$, and we observe that the function $r \mapsto\left|\gamma_{n}(r)\right|$ reaches its minimum at $r=r_{n}$, where $r_{n}=\Omega_{n}^{-1}\left(b_{n}\right)$. As $b_{n} \rightarrow 0$, it is clear that $r_{n} \rightarrow \infty$ as $n \rightarrow \infty$, and since $r^{2} \Omega_{n}(r)$ converges uniformly on $\mathbb{R}_{+}$to $r^{2} \Omega(r)$ (by the results of Section 6.4), we even have $\lim _{n \rightarrow \infty} r_{n}^{2} b_{n}=\lim _{r \rightarrow \infty} r^{2} \Omega(r)=\Gamma>0$, hence

$$
r_{n}^{2}=\frac{\Gamma_{n}}{b_{n}} \quad \forall n \in \mathbb{N}, \quad \text { where } \quad \Gamma_{n} \underset{n \rightarrow \infty}{\longrightarrow} \Gamma .
$$

Similarly we have $\Omega_{n}^{\prime}\left(r_{n}\right)=-d_{n} b_{n}^{3 / 2}$ for all $n \in \mathbb{N}$, where $d_{n} \rightarrow 2 \Gamma^{-1 / 2}$ as $n \rightarrow \infty$.

Eq. (4.20) has asymptotically constant coefficients, in the sense that $\mathcal{A}^{\prime}(r) / \mathcal{A}(r)=\mathcal{O}\left(r^{-3}\right)$ and $\mathcal{D}_{n}(r) \rightarrow k^{2}$ as $r \rightarrow \infty$. However, in general, the convergence of $\mathcal{D}_{n}(r)$ toward its limit $k^{2}$ is not uniform with respect to $n \in \mathbb{N}$, because of the "critical layer" that may occur at $r=r_{n}$. Indeed, if we expand the expression $\gamma_{n}(r)$ around that point, we obtain to leading order

$$
\gamma_{n}(r)=\Omega_{n}(r)-\Omega_{n}\left(r_{n}\right)-i a_{n} \approx \Omega_{n}^{\prime}\left(r_{n}\right)\left(r-r_{n}+i c_{n}\right),
$$

where

$$
c_{n}=-\frac{a_{n}}{\Omega_{n}^{\prime}\left(r_{n}\right)}=\frac{1}{d_{n}} \frac{a_{n}}{b_{n}^{3 / 2}} .
$$

It follows that, for $r$ close to $r_{n}$,

$$
\frac{\Phi_{n}(r)}{\gamma_{n}(r)^{2}} \approx \frac{J_{n}\left(r_{n}\right)}{\left(r-r_{n}+i c_{n}\right)^{2}}, \quad \text { where } \quad J_{n}\left(r_{n}\right)=\frac{\Phi_{n}\left(r_{n}\right)}{\Omega_{n}^{\prime}\left(r_{n}\right)^{2}}
$$

We know that $J_{n}\left(r_{n}\right) \rightarrow J(\infty)$ as $n \rightarrow \infty$, where $0<J(\infty)<\frac{m^{2}}{4 k^{2}}$ (by (4.9) and because $r_{*}<\infty$ ). Thus, the term involving $\gamma_{n}(r)^{-2}$ in (4.21) converges to zero as $r \rightarrow \infty$ uniformly in $n \in \mathbb{N}$ only if $c_{n} \rightarrow \infty$ as $n \rightarrow \infty$, which is the case if $a_{n} \gg b_{n}^{3 / 2}$. Otherwise, that term plays an important role and has to be taken into account.

Our strategy is thus to compare for large $r$ the solutions of (4.20) with those of the simplified equation

$$
w_{n}^{\prime \prime}(r)-\left(k^{2}-\frac{k^{2}}{m^{2}} \frac{J_{n}\left(r_{n}\right)}{\left(r-r_{n}+i c_{n}\right)^{2}}\right) w_{n}(r)=0,
$$

which can be solved explicitly in terms of modified Bessel functions. In particular, the unique solution of (4.25) such that $w_{n}(r) \sim e^{-k\left(r-r_{n}\right)}$ as $r \rightarrow \infty$ is given by

$$
w_{n}(r)=\chi_{n}(r):=\sqrt{\frac{2}{\pi}}\left(r-r_{n}+i c_{n}\right)^{1 / 2} K_{\nu_{n}}\left(k\left(r-r_{n}+i c_{n}\right)\right), \quad r>0,
$$


where $K_{\nu}$ is the modified Bessel function of the second kind [1, Section 9.6], and the parameter $\nu_{n} \in\left(0, \frac{1}{2}\right)$ is determined by the relation

$$
\nu_{n}^{2}=\frac{1}{4}-\frac{k^{2}}{m^{2}} J_{n}\left(r_{n}\right) .
$$

To perform a rigorous analysis, we rewrite (4.20) in the equivalent form

$$
w_{n}^{\prime \prime}(r)+\frac{\mathcal{A}^{\prime}(r)}{\mathcal{A}(r)} w_{n}^{\prime}(r)-\left(k^{2}-\frac{k^{2}}{m^{2}} \frac{J_{n}\left(r_{n}\right)}{\left(r-r_{n}+i c_{n}\right)^{2}}+\mathcal{R}_{n}(r)\right) w_{n}(r)=0,
$$

where the remainder $\mathcal{R}_{n}$ is defined by

$$
\mathcal{R}_{n}(r)=\mathcal{D}_{n}(r)-k^{2}+\frac{k^{2}}{m^{2}} \frac{J_{n}\left(r_{n}\right)}{\left(r-r_{n}+i c_{n}\right)^{2}}, \quad r>0 .
$$

The idea is now to look for a solution of (4.28) in the form

$$
w_{n}(r)=f_{n}(r) \chi_{n}(r), \quad r>0,
$$

where $\chi_{n}$ is as in (4.26), and $f_{n}(r) \rightarrow 1$ as $r \rightarrow \infty$. The equation satisfied by $f_{n}$ is easily found to be

$$
\frac{\mathrm{d}}{\mathrm{d} r}\left(\mathcal{A}(r) \chi_{n}(r)^{2} f_{n}^{\prime}(r)\right)=\left(\mathcal{A}(r) \chi_{n}(r)^{2} \mathcal{R}_{n}(r)-\mathcal{A}^{\prime}(r) \chi_{n}(r) \chi_{n}^{\prime}(r)\right) f_{n}(r), \quad r>0 .
$$

Integrating both sides over $(r, \infty)$, we first obtain

$$
f_{n}^{\prime}(r)=-\frac{1}{\mathcal{A}(r) \chi_{n}(r)^{2}} \int_{r}^{\infty}\left(\mathcal{A}(s) \chi_{n}(s)^{2} \mathcal{R}_{n}(s)-\mathcal{A}^{\prime}(s) \chi_{n}(s) \chi_{n}^{\prime}(s)\right) f_{n}(s) \mathrm{d} s,
$$

and a second integration, combined with an application of Fubini's theorem, gives the representation formula

$$
f_{n}(r)=1+\int_{r}^{\infty} \mathcal{K}_{n}(r, s) f_{n}(s) \mathrm{d} s
$$

where the integral kernel $\mathcal{K}_{n}(r, s)$ has the following expression:

$$
\mathcal{K}_{n}(r, s)=\left(\mathcal{A}(s) \chi_{n}(s)^{2} \mathcal{R}_{n}(s)-\mathcal{A}^{\prime}(s) \chi_{n}(s) \chi_{n}^{\prime}(s)\right) \int_{r}^{s} \frac{1}{\mathcal{A}(t) \chi_{n}(t)^{2}} \mathrm{~d} t .
$$

We now use the following estimate, whose proof is postponed to Section 6.5:

Lemma 4.6. For any $\delta \in(0,1)$, there exists a constant $C>0$ such that

$$
\sup _{r \geq \delta r_{n}} \int_{r}^{\infty}\left|\mathcal{K}_{n}(r, s)\right| \mathrm{d} s \leq C b_{n}^{1 / 2}\left(1+\log _{+} \frac{1}{b_{n}}\right),
$$

for all sufficiently large $n \in \mathbb{N}$, where $\log _{+}(x)=\max (\log (x), 0)$.

Assuming (4.32) for the moment, we easily deduce that the solution of (4.30) satisfies,

$$
\sup _{r \geq \delta r_{n}}\left|f_{n}(r)-1\right| \leq C b_{n}^{1 / 2}\left(1+\log _{+} \frac{1}{b_{n}}\right) \underset{n \rightarrow \infty}{\longrightarrow} 0 .
$$

Also, differentiating (4.30) and using similar estimates as in the proof of Lemma 4.6, we obtain

$$
\sup _{r \geq \delta r_{n}}\left|f_{n}^{\prime}(r)\right| \leq C b_{n}^{1 / 2}\left(1+\log _{+} \frac{b_{n}}{a_{n}}\right) .
$$


These estimates imply that the solution $w_{n}=f_{n} \chi_{n}$ of (4.28) is very close for large $n \in \mathbb{N}$ to its approximation $\chi_{n}$ defined by (4.26), uniformly on the interval $\left[\delta r_{n}, \infty\right)$ for any (small) $\delta>0$. In particular, in view of (6.44), there exist positive constants $C_{1}, C_{2}$ such that

$$
\begin{array}{ll}
\left|w_{n}(r)\right| \leq C_{1} e^{-k\left(r-r_{n}\right)} & \text { for } \quad r \geq r_{n}-1 \\
\left|w_{n}(r)\right| \geq C_{2} e^{-k\left(r-r_{n}\right)} & \text { for } \quad \delta r_{n} \leq r \leq(1-\delta) r_{n} .
\end{array}
$$

To reach the desired contradiction, we now show that these bounds are incompatible with identity (3.11), which has to be satisfied for all $n \in \mathbb{N}$ by the function $u_{n}(r)=r^{-1 / 2} w_{n}(r)$. In terms of $w_{n}$, identity (3.11) becomes

$$
\int_{0}^{\infty}\left\{\frac{2 \mathcal{A}(r)\left(b_{n}-\Omega_{n}\right)}{\left(a_{n}^{2}+\left(\Omega_{n}-b_{n}\right)^{2}\right)^{2}} \frac{k^{2}}{m^{2}} \Phi_{n}(r)+\frac{r}{a_{n}^{2}+\left(\Omega_{n}-b_{n}\right)^{2}} \partial_{r}\left(\frac{W_{n}(r)}{m^{2}+k^{2} r^{2}}\right)\right\}\left|w_{n}(r)\right|^{2} \mathrm{~d} r=0 .
$$

The second term in the integrand is obviously negative, because $W_{n}(r)$ is a decreasing function of $r$. It follows that $\mathcal{I}_{n, 1}+\mathcal{I}_{n, 2} \leq \mathcal{I}_{n, 3}$, where

$$
\begin{aligned}
& \mathcal{I}_{n, 1}=\int_{0}^{r_{n}-1} \frac{\mathcal{A}(r)\left(\Omega_{n}(r)-b_{n}\right)}{\left(a_{n}^{2}+\left(\Omega_{n}(r)-b_{n}\right)^{2}\right)^{2}} \Phi_{n}(r)\left|w_{n}(r)\right|^{2} \mathrm{~d} r, \\
& \mathcal{I}_{n, 2}=\int_{r_{n}-1}^{r_{n}+1} \frac{\mathcal{A}(r)\left(\Omega_{n}(r)-b_{n}\right)}{\left(a_{n}^{2}+\left(\Omega_{n}(r)-b_{n}\right)^{2}\right)^{2}} \Phi_{n}(r)\left|w_{n}(r)\right|^{2} \mathrm{~d} r, \\
& \mathcal{I}_{n, 3}=\int_{r_{n}+1}^{\infty} \frac{\mathcal{A}(r)\left(b_{n}-\Omega_{n}(r)\right)}{\left(a_{n}^{2}+\left(\Omega_{n}(r)-b_{n}\right)^{2}\right)^{2}} \Phi_{n}(r)\left|w_{n}(r)\right|^{2} \mathrm{~d} r .
\end{aligned}
$$

Since $\Omega_{n}(r)-b_{n}$ is positive for $r<r_{n}$ and negative for $r>r_{n}$, the quantities $\mathcal{I}_{n, 1}$ and $\mathcal{I}_{n, 3}$ are positive, whereas $\mathcal{I}_{n, 2}$ has no obvious sign.

We first estimate $\mathcal{I}_{n, 3}$. As $b_{n}-\Omega_{n}(r)=\Omega_{n}\left(r_{n}\right)-\Omega_{n}(r) \geq \Omega_{n}\left(r_{n}\right)-\Omega_{n}\left(r_{n}+1\right) \geq C\left|\Omega^{\prime}\left(r_{n}\right)\right|$ for $r \geq r_{n}+1$, we have

$$
\frac{\left(b_{n}-\Omega_{n}(r)\right) \Phi_{n}(r)}{\left(a_{n}^{2}+\left(\Omega_{n}(r)-b_{n}\right)^{2}\right)^{2}} \leq \frac{C \Phi_{n}(r)}{\left|\Omega^{\prime}\left(r_{n}\right)\right|^{3}} \leq \frac{C J_{n}(r)}{\left|\Omega^{\prime}\left(r_{n}\right)\right|} \leq C b_{n}^{-3 / 2}, \quad r \geq r_{n}+1,
$$

and using (4.35), (4.38) we deduce that $\mathcal{I}_{n, 3} \leq C b_{n}^{-3 / 2}$. Next, we bound $\mathcal{I}_{n, 1}$ from below by restricting the integral in (4.36) to the region where $\delta r_{n} \leq r \leq(1-\delta) r_{n}$, for some small $\delta>0$. In that region we have $\epsilon_{1} b_{n} \leq \Omega_{n}(r)-b_{n} \leq \epsilon_{2} b_{n}$ and $\left|\Omega_{n}^{\prime}(r)\right| \geq \epsilon_{3} b_{n}^{3 / 2}$ for some $\epsilon_{1}, \epsilon_{2}, \epsilon_{3}>0$ depending on $\delta$, hence

$$
\frac{\left(\Omega_{n}(r)-b_{n}\right) \Phi_{n}(r)}{\left(a_{n}^{2}+\left(\Omega_{n}(r)-b_{n}\right)^{2}\right)^{2}} \geq \frac{\epsilon_{1} b_{n} \Omega_{n}^{\prime}(r)^{2} J_{n}(r)}{\left(a_{n}^{2}+\left(\epsilon_{2} b_{n}\right)^{2}\right)^{2}} \geq \frac{C b_{n}^{4}}{\left(a_{n}^{2}+b_{n}^{2}\right)^{2}}, \quad \delta r_{n} \leq r \leq(1-\delta) r_{n},
$$

and using (4.35) we deduce that

$$
\mathcal{I}_{n, 1} \geq \int_{\delta r_{n}}^{(1-\delta) r_{n}} \frac{\mathcal{A}(r)\left(b_{n}-\Omega_{n}(r)\right)}{\left(a_{n}^{2}+\left(\Omega_{n}(r)-b_{n}\right)^{2}\right)^{2}} \Phi_{n}(r)\left|w_{n}(r)\right|^{2} \mathrm{~d} r \geq \frac{C b_{n}^{4}}{\left(a_{n}^{2}+b_{n}^{2}\right)^{2}} e^{2 k(1-\delta) r_{n}} .
$$

Finally, when $\left|r-r_{n}\right| \leq 1$, we have $\Omega_{n}(r)-b_{n} \approx\left(r-r_{n}\right) \Omega_{n}^{\prime}\left(r_{n}\right)$ hence

$$
\frac{\left|\Omega_{n}(r)-b_{n}\right| \Phi_{n}(r)}{\left(a_{n}^{2}+\left(\Omega_{n}(r)-b_{n}\right)^{2}\right)^{2}} \leq \frac{C\left|\Omega^{\prime}\left(r_{n}\right)\right|^{3}\left|r-r_{n}\right|}{\left(a_{n}^{2}+\Omega^{\prime}\left(r_{n}\right)^{2}\left(r-r_{n}\right)^{2}\right)^{2}} J_{n}\left(r_{n}\right) \leq \frac{C}{\left|\Omega^{\prime}\left(r_{n}\right)\right|} \frac{\left|r-r_{n}\right|}{\left(\left(r-r_{n}\right)^{2}+c_{n}^{2}\right)^{2}},
$$


and using (4.35) we obtain the crude estimate

$$
\left|\mathcal{I}_{n, 2}\right| \leq C \int_{r_{n}-1}^{r_{n}+1} \frac{b_{n}^{-3 / 2}\left|r-r_{n}\right|}{\left(\left(r-r_{n}\right)^{2}+c_{n}^{2}\right)^{2}} \mathrm{~d} r \leq C \int_{-\infty}^{\infty} \frac{b_{n}^{-3 / 2}|x|}{\left(x^{2}+c_{n}^{2}\right)^{2}} \mathrm{~d} x \leq \frac{C b_{n}^{-3 / 2}}{c_{n}^{2}} \leq \frac{C b_{n}^{3 / 2}}{a_{n}^{2}} .
$$

As $\mathcal{I}_{n, 1} \leq\left|\mathcal{I}_{n, 2}\right|+\mathcal{I}_{n, 3}$, the estimates obtained so far show that

$$
\frac{C_{3} b_{n}^{4}}{\left(a_{n}^{2}+b_{n}^{2}\right)^{2}} e^{2 k(1-\delta) r_{n}} \leq C_{4} b_{n}^{-3 / 2}\left(1+\frac{b_{n}^{3}}{a_{n}^{2}}\right)
$$

for some positive constants $C_{3}, C_{4}$. If $a_{n} \geq b_{n}$ for a sequence of integers $n$, then multiplying both sides of (4.39) by $\left(a_{n}^{2}+b_{n}^{2}\right)^{2}$ we clearly obtain an inequality that cannot be satisfied for large $n$ if $a_{n} \rightarrow 0$. Thus we can assume that $a_{n} \leq b_{n}$ for all $n \in \mathbb{N}$, in which case (4.39) implies that

$$
C_{3} b_{n}^{3 / 2} e^{2 k(1-\delta) r_{n}} \leq 4 C_{4}\left(1+\frac{b_{n}^{3}}{a_{n}^{2}}\right), \quad \text { hence } \quad a_{n}^{2} \leq C b_{n}^{3 / 2} e^{-2 k(1-\delta) r_{n}} .
$$

Since $r_{n}=\mathcal{O}\left(b_{n}^{-1 / 2}\right)$, this means that $a_{n}$ is exponentially small when compared to $b_{n}$. With this information at hand, it is possible to compute explicitly the quantity $\mathcal{I}_{n, 2}$ to leading order as $n \rightarrow \infty$. Indeed, in this parameter regime, the main contribution to the integral (4.37) comes from an extremely small neighborhood of the critical point $r_{n}$, so that

$$
\begin{aligned}
\mathcal{I}_{n, 2} & \approx-\frac{\mathcal{A}\left(r_{n}\right) \Phi_{n}\left(r_{n}\right)}{\left|\Omega^{\prime}\left(r_{n}\right)\right|^{3}} \int_{r_{n}-1}^{r_{n}+1} \frac{r-r_{n}}{\left(c_{n}^{2}+\left(r-r_{n}\right)^{2}\right)^{2}}\left|w_{n}(r)\right|^{2} \mathrm{~d} r \\
& \approx-\frac{2 \mathcal{A}\left(r_{n}\right) J_{n}\left(r_{n}\right)}{\pi\left|\Omega^{\prime}\left(r_{n}\right)\right|} \int_{r_{n}-1}^{r_{n}+1} \frac{r-r_{n}}{\left(c_{n}^{2}+\left(r-r_{n}\right)^{2}\right)^{3 / 2}}\left|K_{\nu_{n}}\left(k\left(r-r_{n}+i c_{n}\right)\right)\right|^{2} \mathrm{~d} r
\end{aligned}
$$

where in the second line we used the fact that the solution $w_{n}$ of (4.20) is well approximated for large $n$ by the function $\chi_{n}$ in (4.26) in view of (4.33) and (4.34). Now, an explicit calculation which is reproduced in Section 6.6 shows that, for any $\nu \in(0,1 / 2)$ and any $\epsilon>0$,

$$
\mathcal{J}_{\nu}:=\lim _{a \rightarrow 0_{+}} \int_{-\epsilon}^{\epsilon} \frac{-a x}{\left(a^{2}+x^{2}\right)^{3 / 2}}\left|K_{\nu}(x+i a)\right|^{2} \mathrm{~d} x=\frac{2 \pi \cos (\nu \pi)}{1-4 \nu^{2}} .
$$

Assuming (4.42) for the moment, we deduce from (4.41) that

$$
\mathcal{I}_{n, 2} \approx \frac{2 \mathcal{A}\left(r_{n}\right) J_{n}\left(r_{n}\right)}{\pi\left|\Omega^{\prime}\left(r_{n}\right)\right|} \frac{\mathcal{J}_{\nu_{n}}}{c_{n}}, \quad \text { as } n \rightarrow \infty,
$$

which means in particular that $\mathcal{I}_{n, 2}>0$ when $n$ is sufficiently large. Thus, for large $n$ we must have

$$
C_{5} e^{2 k(1-\delta) r_{n}} \leq \mathcal{I}_{n, 1} \leq \mathcal{I}_{n, 1}+\mathcal{I}_{n, 2} \leq \mathcal{I}_{n, 3} \leq C_{6} r_{n}^{3},
$$

for some positive constants $C_{5}, C_{6}$, which is clearly impossible since $r_{n} \rightarrow \infty$ as $n \rightarrow \infty$. So we have reached a contradiction in that case too.

\subsection{The situation $r_{*}=\bar{r}=\infty$ is excluded}

Having exhausted all possibilities for which $r_{*}<\infty$, we finally consider the case where $r_{*}=\infty$. According to (4.7), this occurs if and only if $J(\infty) \geq m^{2} /\left(4 k^{2}\right)$. Of course, we can assume that $\bar{r}=\infty$, because when $\bar{r}<\infty$ a contradiction has already been obtained in Section 4.4 or 4.5. If $J(\infty)>m^{2} /\left(4 k^{2}\right)$, then $J_{n}(r)>J_{n}(\infty)>m^{2} /\left(4 k^{2}\right)$ for all $r>0$ when $n$ is sufficiently large, 
and in that situation we know from Proposition 3.4 that the operator $L_{m, k}^{n}$ has no eigenvalue outside the imaginary axis. However, if $J(\infty)=m^{2} /\left(4 k^{2}\right)$, it is possible that $J_{n}(\infty)<m^{2} /\left(4 k^{2}\right)$ for all $n \in \mathbb{N}$, in which case we cannot obtain a contradiction directly from Proposition 3.4. In that situation, we must have $J_{n}\left(r_{n}\right) \rightarrow m^{2} /\left(4 k^{2}\right)$ as $n \rightarrow \infty$. Two possibilities can occur :

1. If $J_{n}\left(r_{n}\right)<m^{2} /\left(4 k^{2}\right)$ for a sequence of integers $n$, we can get a contradiction by following exactly the same lines as in Section 4.8, the only difference being that the indices $\nu_{n}$ defined by (4.27) now converge to zero as $n \rightarrow \infty$. This is harmless because, as is observed for instance in Remark 6.7 below, all estimates we need hold uniformly in the limit where $\nu \rightarrow 0$. We leave the details to the reader.

2. If instead $J_{n}\left(r_{n}\right) \geq m^{2} /\left(4 k^{2}\right)$ for all $n \in \mathbb{N}$, we shall prove that the quadratic form given by the left-hand side of (4.11) is positive definite for sufficiently large values of $n$, so that (4.11) gives the desired contradiction. To do that, we use the asymptotic expansions

$$
\Omega_{n}(r)=\frac{\Gamma_{n}}{r^{2}}+\mathcal{O}\left(\frac{1}{r^{4}}\right), \quad \Omega_{n}^{\prime}(r)=\frac{-2 \Gamma_{n}}{r^{3}}+\mathcal{O}\left(\frac{1}{r^{5}}\right), \quad J_{n}^{\prime}(r)=o\left(\frac{1}{r}\right), \quad r \rightarrow \infty,
$$

which are established in Section 6.4 and we observe that the integrand in (4.11) is nonnegative outside an interval of the form $\left[r_{n}, r_{n}+\delta_{n}\right]$, where $\delta_{n} \rightarrow 0$ as $n \rightarrow \infty$. Indeed, the integrand is clearly nonnegative for $r \leq r_{n}$, and for $r \geq r_{n}$ we have the lower bound

$$
\frac{\mathcal{A}(r) \Omega_{n}^{\prime}(r)^{2}}{a_{n}^{2}+\left(b_{n}-\Omega_{n}(r)\right)^{2}}\left(\frac{k^{2}}{m^{2}} J_{n}(r)-\frac{1}{4}\right) \geq \frac{1}{m^{2}} \frac{\Omega_{n}^{\prime}(r)^{2}}{\left(b_{n}-\Omega_{n}(r)\right)^{2}}\left(J_{n}(r)-J_{n}\left(r_{n}\right)\right) .
$$

In view of (4.43), the last member of (4.44) is bounded from below by -1 if $r \geq r_{n}+\mathcal{O}\left(\left|J^{\prime}\left(r_{n}\right)\right|\right)$, which proves the claim. If we now restrict the integral in (4.11) to the interval $\left[r_{n}-1, r_{n}+1\right]$, make the change of variable $r=r_{n}+s$, and use the lower bound (4.44), we deduce (after a few obvious simplifications) that it is sufficient for us to show that the quadratic form

$$
\mathfrak{Q}_{n}(v):=\int_{-1}^{1}\left\{\left|\partial_{s} v\right|^{2}+\left(k^{2}-\frac{\varepsilon_{n} s}{c_{n}^{2}+s^{2}}\right)|v|^{2}\right\} \mathrm{d} s, \quad k>0,
$$

is positive on $H^{1}([-1,1], \mathrm{d} s)$ for all $c_{n} \neq 0$ and all sufficiently small $\varepsilon_{n}>0$. This in turn is an easy consequence of the Sobolev embedding theorem. Indeed, decomposing $v=v(0) \chi+w$ where $\chi:[-1,1] \rightarrow[0,1]$ is smooth, even, and satisfies $\chi(0)=1$, we first observe that

$$
\int_{-1}^{1} \frac{\varepsilon_{n} s}{c_{n}^{2}+s^{2}}|v(0)|^{2} \chi^{2}(s) \mathrm{d} s=0,
$$

by symmetry. Moreover, as $w(0)=0$ by construction, we have $|w(s)|^{2} \leq C|s|\|v\|_{H^{1}}^{2}$. Combining these observations, we deduce that

$$
\left.\left|\int_{-1}^{1} \frac{\varepsilon_{n} s}{c_{n}^{2}+s^{2}}\right| v\right|^{2} \mathrm{~d} s \mid \leq C \varepsilon_{n}\|v\|_{H^{1}}^{2}
$$

where the constant $C>0$ is independent of $n$. The quadratic form (4.45) is thus positive if $\varepsilon_{n}$ is sufficiently small, and we deduce that (4.11) cannot be satisfied. This concludes the contradiction argument initiated in Section 4.1, hence also the proof of Theorem 1.3.

\section{Uniform resolvent estimates}

This section is devoted to the proof of Theorem 1.6. Given any $s \in \mathbb{C}$ with $\operatorname{Re}(s) \neq 0$, we already know from Theorem 1.3 that the resolvent operator $\left(s-L_{m, k}\right)^{-1}$ is bounded in the space $X_{m, k}$ 
for all $m \in \mathbb{Z}$ and all nonzero $k \in \mathbb{R}$. It remains to show that, for any $k_{0}>0$, the resolvent norm $\left\|\left(s-L_{m, k}\right)^{-1}\right\|$ is uniformly bounded for all $m \in \mathbb{Z}$ and all nonzero $k \in \mathbb{Z} k_{0}$.

Given $m \in \mathbb{Z}, k \neq 0$, and $\omega, f \in X_{m, k}$, the resolvent equation $\left(s-L_{m, k}\right) \omega=f$ takes the form

$$
\begin{aligned}
& \gamma(r) \omega_{r}=i k W(r) u_{r}+f_{r}, \\
& \gamma(r) \omega_{\theta}=i k W(r) u_{\theta}+r \Omega^{\prime}(r) \omega_{r}+f_{\theta}, \\
& \gamma(r) \omega_{z}=i k W(r) u_{z}-W^{\prime}(r) u_{r}+f_{z},
\end{aligned}
$$

where $\gamma(r)=s+i m \Omega(r)$. Here and in what follows, we assume that $\operatorname{Re}(s) \neq 0$, which implies that $|\gamma(r)| \geq|\operatorname{Re}(s)|>0$ for all $r>0$. In (5.1), it is understood that the velocity $u$ is obtained from the vorticity $\omega$ by the Biot-Savart formula in the Fourier subspace indexed by $m$ and $k$, see Section 6.1 below.

Proceeding as in the derivation of the eigenvalue equation (2.16) in Section 2, we can transform the resolvent system (5.2) into a single equation for the radial velocity $u_{r}$. After some calculations, we obtain the differential equation

$$
-\partial_{r}\left(\mathcal{A}(r) \partial_{r}^{*} u_{r}\right)+\mathcal{B}(r) u_{r}=\mathcal{F}(r),
$$

where the coefficients in the left-hand side are as in (3.3) :

$$
\mathcal{A}(r)=\frac{r^{2}}{m^{2}+k^{2} r^{2}}, \quad \mathcal{B}(r)=1+\frac{k^{2}}{\gamma(r)^{2}} \mathcal{A}(r) \Phi(r)+\frac{i m r}{\gamma(r)} \partial_{r}\left(\frac{W(r)}{m^{2}+k^{2} r^{2}}\right),
$$

and the right-hand side takes the form:

$$
\mathcal{F}(r)=-\partial_{r}\left(\frac{m}{k r \gamma(r)} \mathcal{A}(r) f_{r}\right)-\frac{i m^{2}}{k \gamma(r)^{2}} \frac{W(r)}{m^{2}+k^{2} r^{2}} f_{r}-\frac{i}{k \gamma(r)} f_{\theta}+\frac{2 i \Omega(r)}{k \gamma(r)^{2}} f_{z} .
$$

Of course, if $f=0$, then $\mathcal{F}=0$ and (5.2) reduces to (2.16). The following result will be useful to estimate the solutions of (5.2) when $|k|$ is large.

Lemma 5.1. For any $m \in \mathbb{Z}$ and any $s \in \mathbb{C}$ with $\operatorname{Re}(s) \neq 0$, there exists a positive constant $C=C(m, s)$ such that, for any $k \neq 0$ and any $f \in X_{m, k}$, the solution $u_{r}$ of (5.2) satisfies

$$
\left\|\mathcal{A}^{1 / 2} \partial_{r}^{*} u_{r}\right\|_{L^{2}}+\left\|u_{r}\right\|_{L^{2}} \leq \frac{C(m, s)}{|k|}\left(\left\|u_{r}\right\|_{L^{2}}+\|f\|_{L^{2}}\right)
$$

Proof. As in Section 3.3, we set $u_{r}(r)=\gamma(r)^{1 / 2} v(r)$. The new function $v$ satisfies the equation

$$
-\partial_{r}\left(\mathcal{A}(r) \gamma(r) \partial_{r}^{*} v\right)+\mathcal{E}(r) v=\gamma(r)^{1 / 2} \mathcal{F}(r),
$$

where

$$
\begin{aligned}
\mathcal{E}(r) & =\gamma(r) \mathcal{B}(r)-\frac{\gamma^{\prime}(r)}{2}\left(\mathcal{A}^{\prime}(r)-\frac{\mathcal{A}(r)}{r}\right)-\frac{1}{2} \gamma^{\prime \prime}(r) \mathcal{A}(r)+\frac{\gamma^{\prime}(r)^{2} \mathcal{A}(r)}{4 \gamma(r)} \\
& =\gamma(r)+\frac{k^{2}}{\gamma(r)} \mathcal{A}(r) \Phi(r)+\frac{i m r}{2} \partial_{r}\left(\frac{W(r)+2 \Omega(r)}{m^{2}+k^{2} r^{2}}\right)-\frac{m^{2} \Omega^{\prime}(r)^{2}}{4 \gamma(r)} \mathcal{A}(r) .
\end{aligned}
$$

We also observe that

$$
\gamma^{1 / 2} \mathcal{F}=-\partial_{r}\left(\frac{m}{k r \gamma^{1 / 2}} \mathcal{A} f_{r}\right)-\frac{i m^{2}}{2 k \gamma^{3 / 2}} \frac{W+2 \Omega}{m^{2}+k^{2} r^{2}} f_{r}-\frac{i}{k \gamma^{1 / 2}} f_{\theta}+\frac{2 i \Omega}{k \gamma^{3 / 2}} f_{z} .
$$


Without loss of generality, we assume that $a:=\operatorname{Re}(s)>0$. If we multiply both sides of (5.6) by $r \bar{v}$, integrate the resulting equality over $\mathbb{R}_{+}$and take the real part, we obtain the identity

$$
\begin{aligned}
a \int_{0}^{\infty}\left\{\mathcal{A}\left|\partial_{r}^{*} v\right|^{2}+|v|^{2}\right. & \left.+\frac{\mathcal{A}}{|\gamma|^{2}}\left(k^{2} \Phi-\frac{m^{2} \Omega^{\prime 2}}{4}\right)|v|^{2}\right\} r \mathrm{~d} r=\operatorname{Re} \int_{0}^{\infty}\left(\partial_{r}^{*} \bar{v}\right) \frac{m \mathcal{A}}{k r \gamma^{1 / 2}} f_{r} r \mathrm{~d} r \\
& +\operatorname{Re} \int_{0}^{\infty} \bar{v}\left(-\frac{i m^{2}}{2 k \gamma^{3 / 2}} \frac{W+2 \Omega}{m^{2}+k^{2} r^{2}} f_{r}-\frac{i}{k \gamma^{1 / 2}} f_{\theta}+\frac{2 i \Omega}{k \gamma^{3 / 2}} f_{z}\right) r \mathrm{~d} r
\end{aligned}
$$

Keeping in mind that $\Phi(r) \geq 0,|\gamma(r)| \geq a$, and $0<\mathcal{A}(r) \leq \min \left(1 / k^{2}, r^{2} / m^{2}\right)$, we can estimate the various terms in a straightforward way, and we arrive at the inequality

$$
\begin{aligned}
a\left(\left\|\mathcal{A}^{1 / 2} \partial_{r}^{*} v\right\|_{L^{2}}^{2}+\|v\|_{L^{2}}^{2}\right) \leq & \frac{C m^{2}}{a k^{2}}\|v\|_{L^{2}}^{2}+\frac{C}{a^{1 / 2}|k|}\left\|\mathcal{A}^{1 / 2} \partial_{r}^{*} v\right\|_{L^{2}}\left\|f_{r}\right\|_{L^{2}} \\
& +\frac{C}{a^{3 / 2}|k|}\|v\|_{L^{2}}\left(\left\|f_{r}\right\|_{L^{2}}+a\left\|f_{\theta}\right\|_{L^{2}}+\left\|f_{z}\right\|_{L^{2}}\right)
\end{aligned}
$$

where $C>0$ is a universal constant. Applying now Young's inequality, we conclude that

$$
\left\|\mathcal{A}^{1 / 2} \partial_{r}^{*} v\right\|_{L^{2}}+\|v\|_{L^{2}} \leq \frac{C(m, a)}{|k|}\left(\|v\|_{L^{2}}+\|f\|_{L^{2}}\right)
$$

where the constant depends only on $m$ and $a$.

Finally, we return to the original function $u_{r}(r)=\gamma(r)^{1 / 2} v(r)$. As $|\gamma(r)| \leq|s|+|m|$ and

$$
\mathcal{A}^{1 / 2} \partial_{r}^{*} u_{r}=\mathcal{A}^{1 / 2} \gamma^{1 / 2}\left(\partial_{r}^{*} v+\frac{i m r \Omega^{\prime}}{2 r \gamma} v\right)
$$

we have $\left|\mathcal{A}^{1 / 2} \partial_{r}^{*} u_{r}\right|+\left|u_{r}\right| \leq C(m, s)\left(\left|\mathcal{A}^{1 / 2} \partial_{r}^{*} v\right|+|v|\right)$. Thus the desired inequality (5.5) follows immediately from (5.9).

Equipped with this lemma, we now establish the main result of this section.

Proposition 5.2. Fix any $k_{0}>0$. For any $s \in \mathbb{C}$ with $\operatorname{Re}(s) \neq 0$, there exists a constant $C=C\left(s, k_{0}\right)$ such that, for all $m \in \mathbb{Z}$ and all nonzero $k \in \mathbb{Z} k_{0}$, the following estimate holds for all $f \in X_{m, k}$ :

$$
\left\|\left(s-L_{m, k}\right)^{-1} f\right\|_{L^{2}} \leq C\|f\|_{L^{2}} .
$$

Proof. We proceed by contradiction. If (5.10) does not hold, there exist sequences $\left(m_{n}\right)$ in $\mathbb{Z},\left(k_{n}\right)$ in $\mathbb{Z}^{*} k_{0}$, and $\omega^{(n)}, f^{(n)}$ in $X_{m, k}$ such that $\left\|\omega^{(n)}\right\|_{L^{2}}=1$ for all $n \in \mathbb{N},\left\|f^{(n)}\right\|_{L^{2}} \rightarrow 0$ as $n \rightarrow \infty$, and $\left(s-L_{m, k}\right) \omega^{(n)}=f^{(n)}$ for all $n \in \mathbb{N}$, namely

$$
\begin{aligned}
& \left(s+i m_{n} \Omega(r)\right) \omega_{r}^{(n)}=i k_{n} W(r) u_{r}^{(n)}+f_{r}^{(n)} \\
& \left(s+i m_{n} \Omega(r)\right) \omega_{\theta}^{(n)}=i k_{n} W(r) u_{\theta}^{(n)}+r \Omega^{\prime}(r) \omega_{r}^{(n)}+f_{\theta}^{(n)} \\
& \left(s+i m_{n} \Omega(r)\right) \omega_{z}^{(n)}=i k_{n} W(r) u_{z}^{(n)}-W^{\prime}(r) u_{r}^{(n)}+f_{z}^{(n)} .
\end{aligned}
$$

Step 1. We first show that the sequence $\left(m_{n}\right)$ is bounded. Indeed, if this is not the case, we can assume (after extracting a subsequence) that $\left|m_{n}\right| \rightarrow \infty$ as $n \rightarrow \infty$. In view of the first equation in (5.11), this implies that

$$
\left\|\omega_{r}^{(n)}\right\|_{L^{2}} \leq\left\|\frac{i k_{n} W u_{r}^{(n)}}{s+i m_{n} \Omega}\right\|_{L^{2}}+\left\|\frac{f_{r}^{(n)}}{s+i m_{n} \Omega}\right\|_{L^{2}} \underset{n \rightarrow \infty}{\longrightarrow} 0 .
$$


Indeed, we know from Proposition 6.1 that $\left\|k_{n} u^{(n)}\right\|_{L^{2}} \leq C\left\|\omega^{(n)}\right\|_{L^{2}} \leq C$ for all $n \in \mathbb{N}$, so that

$$
\left\|\frac{i k_{n} W u_{r}^{(n)}}{s+i m_{n} \Omega}\right\|_{L^{2}} \leq\left\|\frac{W}{s+i m_{n} \Omega}\right\|_{L^{\infty}}\left\|k_{n} u_{r}^{(n)}\right\|_{L^{2}} \leq C\left\|\frac{W}{s+i m_{n} \Omega}\right\|_{L^{\infty}} \underset{n \rightarrow \infty}{\longrightarrow} 0,
$$

and the last term in (5.12) is bounded by $|\operatorname{Re}(s)|^{-1}\left\|f_{r}^{(n)}\right\|_{L^{2}}$, a quantity that converges to zero as $n \rightarrow \infty$ by assumption. Once (5.12) is known, the same argument applied to the second equation in (5.11) shows that $\left\|\omega_{\theta}^{(n)}\right\|_{L^{2}} \rightarrow 0$. Finally, we have $\left\|u_{r}^{(n)}\right\|_{L^{2}} \leq k_{0}^{-1}\left\|k_{n} u_{r}^{(n)}\right\|_{L^{2}} \leq C k_{0}^{-1}$, because $\left|k_{n}\right| \geq k_{0}$ for all $n \in \mathbb{N}$. Applying thus the same argument again to the third equation in (5.11), we conclude that $\left\|\omega_{z}^{(n)}\right\|_{L^{2}} \rightarrow 0$, which of course contradicts the hypothesis that $\left\|\omega^{(n)}\right\|_{L^{2}}=1$ for all $n \in \mathbb{N}$. This means that sequence $\left(m_{n}\right)$ must be bounded, and after extracting a subsequence we can therefore assume that there exists an integer $m \in \mathbb{Z}$ such that $m_{n}=m$ for all $n \in \mathbb{N}$.

Step 2. We next show that the sequence $\left(k_{n}\right)$ is bounded. Again, if this is not the case, we can assume after extracting a subsequence that $\left|k_{n}\right| \rightarrow \infty$ as $n \rightarrow \infty$. In that situation, we infer from estimate (5.5) that, for $n$ sufficiently large,

$$
\left|k_{n}\right|\left(\left\|\mathcal{A}_{n}^{1 / 2} \partial_{r}^{*} u_{r}^{(n)}\right\|_{L^{2}}+\left\|u_{r}^{(n)}\right\|_{L^{2}}\right) \leq 2 C(m, s)\left\|f^{(n)}\right\|_{L^{2}} \underset{n \rightarrow \infty}{\longrightarrow} 0 .
$$

Next, we use the relation

$$
k_{n}^{2} \mathcal{A}_{n}\left(\partial_{r}^{*}-\frac{i m W}{r \gamma}\right) u_{r}^{(n)}+i k_{n} u_{z}^{(n)}=\frac{m k_{n}}{r \gamma} \mathcal{A}_{n} f_{r}^{(n)}
$$

which reduces to (2.13) when $k_{n}=k$ and $f_{r}^{(n)}=0$. Invoking (5.13) and using the elementary bounds $0<\mathcal{A}_{n}(r) \leq \min \left(1 / k_{n}^{2}, r^{2} / m^{2}\right)$, we deduce that

$$
\left|k_{n}\right|\left\|u_{z}^{(n)}\right\|_{L^{2}}+|m|\left\|\frac{u_{z}^{(n)}}{r}\right\|_{L^{2}} \underset{n \rightarrow \infty}{\longrightarrow} 0 .
$$

Finally, with the help of the additional relation

$$
\frac{i k_{n} W}{\gamma} u_{r}^{(n)}+i k_{n} u_{\theta}^{(n)}-\frac{i m}{r} u_{z}^{(n)}=-\frac{f_{r}^{(n)}}{\gamma}
$$

which reduces to (2.9) when $k_{n}=k$ and $f_{r}^{(n)}=0$, we find that $\left|k_{n}\right|\left\|u_{\theta}^{(n)}\right\|_{L^{2}} \rightarrow 0$ as $n \rightarrow \infty$ in view of (5.13), (5.14).

Thus, we have shown that $\left|k_{n}\right|\left\|u^{(n)}\right\|_{L^{2}} \rightarrow 0$ as $n \rightarrow \infty$, and considering successively all three lines in (5.11) we easily deduce that

$$
\left\|\omega_{r}^{(n)}\right\|_{L^{2}} \underset{n \rightarrow \infty}{\longrightarrow} 0, \quad\left\|\omega_{\theta}^{(n)}\right\|_{L^{2}} \underset{n \rightarrow \infty}{\longrightarrow} 0, \quad\left\|\omega_{z}^{(n)}\right\|_{L^{2}} \underset{n \rightarrow \infty}{\longrightarrow} 0 .
$$

This of course contradicts the assumption that $\left\|\omega^{(n)}\right\|_{L^{2}}=1$ for all $n \in \mathbb{N}$. The sequence $\left(k_{n}\right)$ must therefore be bounded, and after extracting a subsequence we can assume that $k_{n}=k$ for some fixed $k \in \mathbb{Z}^{*} k_{0}$.

Step 3. Assuming that estimate (5.10) does not hold for some $s \in \mathbb{C}$ with $\operatorname{Re}(s) \neq 0$, we have reached the conclusion that, for some $m \in \mathbb{Z}$ and some $k \neq 0$, the operator $s-L_{m, k}$ has no bounded inverse in $X_{m, k}$, in contradiction with Theorem 1.3. Thus estimate (5.10) must hold, and the proof of Proposition 5.2 is complete. 


\section{Appendix}

\subsection{The Biot-Savart law in cylindrical coordinates}

The Biot-Savart law defines the velocity field $u=\left(u_{r}, u_{\theta}, u_{z}\right)$ in terms of the vorticity vector $\omega=\left(\omega_{r}, \omega_{\theta}, \omega_{z}\right)$, for a fixed value of the angular Fourier mode $m \in \mathbb{Z}$ and of the vertical wave number $k \in \mathbb{R}$. The velocity is determined by the linear relations

$$
\omega_{r}=\frac{i m}{r} u_{z}-i k u_{\theta}, \quad \omega_{\theta}=i k u_{r}-\partial_{r} u_{z}, \quad \omega_{z}=\frac{1}{r} \partial_{r}\left(r u_{\theta}\right)-\frac{i m}{r} u_{r},
$$

together with the divergence-free condition

$$
\frac{1}{r} \partial_{r}\left(r u_{r}\right)+\frac{i m}{r} u_{\theta}+i k u_{z}=0 .
$$

These equations have to be solved on the half-line $r>0$, and we require that the velocity field $u_{r} e_{r}+u_{\theta} e_{\theta}+u_{z} e_{z}$ be regular at the origin $r=0$ and decay to zero as $r \rightarrow \infty$. More precisely, if the vorticity $\omega$ is (for instance) compactly supported in $\mathbb{R}_{+}=(0, \infty)$, the following boundary conditions hold for the associated velocity $u$ :

- The horizontal velocities $u_{r}, u_{\theta}$ satisfy the homogeneous Dirichlet condition at $r=0$ if $m=0$ or $|m| \geq 2$, and the homogeneous Neumann condition if $|m|=1$ (or $|m| \geq 3$ ).

- The vertical velocity $u_{z}$ satisfies the homogeneous Dirichlet condition at $r=0$ if $|m| \geq 1$, and the homogeneous Neumann condition if $m=0$ (or $|m| \geq 2$ ).

It is possible to give explicit formulas for the velocity $u$ in terms of the vorticity $\omega$, but the bounds we need in this paper are more conveniently obtained by standard energy estimates. We recall that $\|\cdot\|_{L^{2}}$ denotes the usual norm in the Lebesgue space $L^{2}\left(\mathbb{R}_{+}, r \mathrm{~d} r\right)$.

Proposition 6.1. There exists a constant $C>0$ such that, for any $m \in \mathbb{Z}$ and any $k \in \mathbb{R}$, the following inequality holds

$$
\begin{aligned}
& \left\|\partial_{r} u_{r}\right\|_{L^{2}}^{2}+\left\|\partial_{r} u_{\theta}\right\|_{L^{2}}^{2}+\left\|\partial_{r} u_{z}\right\|_{L^{2}}^{2}+k^{2}\left(\left\|u_{r}\right\|_{L^{2}}^{2}+\left\|u_{\theta}\right\|_{L^{2}}^{2}+\left\|u_{z}\right\|_{L^{2}}^{2}\right) \\
& +\left|m^{2}-1\right|\left(\left\|\frac{u_{r}}{r}\right\|_{L^{2}}^{2}+\left\|\frac{u_{\theta}}{r}\right\|_{L^{2}}^{2}\right)+m^{2}\left\|\frac{u_{z}}{r}\right\|_{L^{2}}^{2} \leq C\left(\left\|\omega_{r}\right\|_{L^{2}}^{2}+\left\|\omega_{\theta}\right\|_{L^{2}}^{2}+\left\|\omega_{z}\right\|_{L^{2}}^{2}\right) .
\end{aligned}
$$

Proof. We assume here for definiteness that $k \neq 0$, but the proof is similar (and in fact simpler) when $k=0$. Without loss of generality, we also suppose that $\omega$ is continuous and compactly supported in $\mathbb{R}_{+}$. We first observe that the vertical velocity $u_{z}$ satisfies the linear elliptic equation

$$
\left(-\partial_{r}^{2}-\frac{1}{r} \partial_{r}+\frac{m^{2}}{r^{2}}+k^{2}\right) u_{z}=\frac{1}{r} \partial_{r}\left(r \omega_{\theta}\right)-\frac{i m}{r} \omega_{r} .
$$

We multiply both sides of (6.4) by $r \bar{u}_{z}$ and integrate the resulting expression over $\mathbb{R}_{+}$. After elementary calculations, we obtain the estimate

$$
\left\|\partial_{r} u_{z}\right\|_{L^{2}}^{2}+\left\|\frac{m}{r} u_{z}\right\|_{L^{2}}^{2}+\left\|k u_{z}\right\|_{L^{2}}^{2} \leq C\left(\left\|\omega_{r}\right\|_{L^{2}}^{2}+\left\|\omega_{\theta}\right\|_{L^{2}}^{2}\right)
$$

where $C>0$ is a universal constant. As $i k u_{r}=\partial_{r} u_{z}+\omega_{\theta}$ and $i k u_{\theta}=\frac{i m}{r} u_{z}-\omega_{r}$, it follows immediately from (6.5) that

$$
\left\|k u_{r}\right\|_{L^{2}}^{2}+\left\|k u_{\theta}\right\|_{L^{2}}^{2} \leq C\left(\left\|\omega_{r}\right\|_{L^{2}}^{2}+\left\|\omega_{\theta}\right\|_{L^{2}}^{2}\right) .
$$


On the other hand, we deduce from (6.2) and the last relation in (6.1) that

$$
\partial_{r} u_{r}+\frac{1}{r}\left(u_{r}+i m u_{\theta}\right)=-i k u_{z}, \quad \partial_{r} u_{\theta}+\frac{1}{r}\left(u_{\theta}-i m u_{r}\right)=\omega_{z} .
$$

We multiply the first equation by $r \partial_{r} \bar{u}_{r}$ and the second one by $r \partial_{r} \bar{u}_{\theta}$. Adding the resulting expressions, taking the real parts, and integrating over $\mathbb{R}_{+}$, we obtain the inequality

$$
\left\|\partial_{r} u_{r}\right\|_{L^{2}}^{2}+\left\|\partial_{r} u_{\theta}\right\|_{L^{2}}^{2} \leq C\left(\left\|k u_{z}\right\|_{L^{2}}^{2}+\left\|\omega_{z}\right\|_{L^{2}}^{2}\right) \leq C\left(\left\|\omega_{r}\right\|_{L^{2}}^{2}+\left\|\omega_{\theta}\right\|_{L^{2}}^{2}+\left\|\omega_{z}\right\|_{L^{2}}^{2}\right) .
$$

If $m= \pm 1$, this concludes the proof of (6.3). Otherwise, we deduce from (6.7) that

$$
\begin{aligned}
& \frac{m^{2}-1}{r} u_{r}=\partial_{r}\left(u_{r}-i m u_{\theta}\right)+i k u_{z}+i m \omega_{z}, \\
& \frac{m^{2}-1}{r} u_{\theta}=\partial_{r}\left(u_{\theta}+i m u_{r}\right)+k m u_{z}-\omega_{z} .
\end{aligned}
$$

If $m=0$ or $|m| \geq 2$, these relations allow us to estimate the $L^{2}$ norm of $u_{r} / r$ and $u_{\theta} / r$ in terms of quantities that are already controlled by (6.5) or (6.8), and we arrive at (6.3).

\subsection{Stability of Rankine's vortex}

We consider here in some detail the particular case of the Rankine vortex (1.7), which is of historical relevance. We do not use the functional framework of Section 2 because, as is clear from (2.3), the linearization $L_{m, k}$ does not define a bounded linear operator on $X_{m, k}$ if the vorticity profile $W$ has a discontinuity. Instead we look for solutions of the eigenvalue equation (2.8) where the velocity field $u$ (and not the vorticity $\omega$ ) belongs to $X_{m, k}$. We always assume that $m \neq 0$ and $k \neq 0$, the other cases being similar and in fact simpler. To avoid the essential spectrum, we also suppose that the spectral parameter $s \in \mathbb{C}$ satisfies $s \neq 0$ and $s+i m \neq 0$.

Following Kelvin's original approach [27], we eliminate the radial velocity $u_{r}$ in the $2 \times 2$ system (2.13) -(2.14) to obtain a closed equation for the vertical velocity $u_{z}$. In the inner region where $0<r<1$, we have $\gamma(r)=\gamma:=s+i m$ and $\Phi(r)=W(r)^{2}=4$, so that $u_{z}$ satisfies the Bessel equation

$$
-\frac{1}{r} \partial_{r}\left(r \partial_{r} u_{z}\right)+\left(\beta^{2}+\frac{m^{2}}{r^{2}}\right) u_{z}=0, \quad \text { where } \quad \beta^{2}=k^{2}\left(1+\frac{4}{\gamma^{2}}\right) .
$$

Since $u_{z}$ is regular at the origin, it follows that $u_{z}(r)=A I_{m}(\beta r)$ for $0<r<1$, where $A \in \mathbb{C}$ and $I_{m}$ is the modified Bessel function of order $m$ [1, Section 9.6]. In the outer region where $r>1$, we have $W(r)=\Phi(r)=0$, and system (2.13)-(2.14) reduces to the (somewhat simpler) Bessel equation

$$
-\frac{1}{r} \partial_{r}\left(r \partial_{r} u_{z}\right)+\left(k^{2}+\frac{m^{2}}{r^{2}}\right) u_{z}=0 .
$$

As $u_{z}(r)$ decays to zero at infinity, we must have $u_{z}(r)=B K_{m}(k r)$ for some $B \in \mathbb{C}$, where $K_{m}$ is again a modified Bessel function.

At the interface $r=1$, both velocities $u_{z}, u_{r}$ are continuous, as can be seen from (2.10) and (2.12). Jump conditions for the first order derivatives can be deduced from system (2.13)-(2.14) and are found to be

$$
\begin{aligned}
& \partial_{r} u_{r}\left(1_{+}\right)=\partial_{r} u_{r}\left(1_{-}\right)-\frac{2 i m}{\gamma} u_{r}(1) \\
& \partial_{r} u_{z}\left(1_{+}\right)=\frac{\gamma^{2}}{\gamma^{2}+4}\left(\partial_{r} u_{z}\left(1_{-}\right)+\frac{2 i m}{\gamma} u_{z}(1)\right)=i k u_{r}(1) .
\end{aligned}
$$


In particular, as $u_{z}(r)=A I_{m}(\beta r)$ for $r<1$ and $u_{z}(r)=B K_{m}(k r)$ for $r>1$, we must have

$$
A I_{m}(\beta)=B K_{m}(k), \quad \frac{A \gamma^{2}}{\gamma^{2}+4}\left(\beta I_{m}^{\prime}(\beta)+\frac{2 i m}{\gamma} I_{m}(\beta)\right)=B k K_{m}^{\prime}(k) .
$$

This linear system has a nontrivial solution $(A, B)$ if and only if

$$
\frac{I_{m}^{\prime}(\beta)}{\beta I_{m}(\beta)}+\frac{2 i m}{\gamma \beta^{2}}=\frac{K_{m}^{\prime}(k)}{k K_{m}(k)},
$$

where we recall that $\gamma=s+i m \neq 0$ and $\beta^{2}=k^{2}\left(1+4 / \gamma^{2}\right)$.

It was already observed by Kelvin that the dispersion relation (6.15) is satisfied for a countable set of purely imaginary values of the spectral parameter $s$. More precisely, if we define $s=-i m b$, so that $\gamma=i m(1-b)$, equality (6.15) holds for a decreasing sequence of values of $b$ accumulating at 1 , and also for an increasing sequence accumulating at 1 , all solutions being contained in the interval $|b-1| \leq 2 /|m|$ [27]. The linearized operator at Rankine's vortex thus has a countable family of purely imaginary eigenvalues (Kelvin modes). However, it is not easy to verify that the dispersion relation (6.15) has no solution when $s \notin i \mathbb{R}$, and there is no such claim in Kelvin's work 4 where only purely imaginary eigenvalues are considered. Thus, contrary to what is often asserted in the literature, stability of Rankine's vortex was not established by Kelvin, and we could not find any further reference where this point is clarified.

Fortunately, it is quite easy to prove spectral stability of Rankine's vortex following the approach of Section 3.3. Indeed, taking into account the particular form of the vorticity profile (1.7), it is straightforward to verify that identity (3.10) becomes

$$
\begin{aligned}
\int_{0}^{\infty}\left(\mathcal{A}(r)\left|\partial_{r}^{*} u_{r}\right|^{2}+\left|u_{r}\right|^{2}\right) r \mathrm{~d} r & \\
& \quad+\int_{0}^{1}\left\{-\frac{4 k^{2} \mathcal{A}(r)}{m^{2} \gamma_{\star}^{2}}+\frac{2 r}{\gamma_{\star}} \partial_{r}\left(\frac{1}{m^{2}+k^{2} r^{2}}\right)\right\}\left|u_{r}\right|^{2} r \mathrm{~d} r=\frac{2 \mathcal{A}(1)}{\gamma_{\star}}\left|u_{r}(1)\right|^{2},
\end{aligned}
$$

see also Remark 6.2 below. Here $\gamma_{\star}=1-b-i a$, so that $\gamma=i m \gamma_{\star}$. We now multiply both sides of (6.16) by $\gamma_{\star}$ and take the imaginary parts. We arrive at the identity

$$
a \int_{0}^{\infty}\left(\mathcal{A}(r)\left|\partial_{r}^{*} u_{r}\right|^{2}+\left|u_{r}\right|^{2}\right) r \mathrm{~d} r+a \int_{0}^{1} \frac{4 k^{2}}{m^{2}} \frac{\mathcal{A}(r)}{(1-b)^{2}+a^{2}}\left|u_{r}\right|^{2} r \mathrm{~d} r=0 .
$$

If $a \neq 0$, it follows from (6.17) that $u_{r} \equiv 0$, hence the eigenvalue equation (2.16) has no nontrivial solution if $s=m(a-i b) \notin i \mathbb{R}$. This proves that the linearized operator at Rankine's vortex has no unstable eigenvalue.

Remark 6.2. Alternatively, one can obtain the relation (6.16) by restricting the eigenvalue equation (2.16) to the open intervals $(0,1)$ and $(1, \infty)$, where the vorticity profile is smooth. On each interval, we multiply (2.16) by $r \bar{u}_{r}$ and we integrate over $r$. If we add the resulting expressions and simplify the boundary terms (which result from partial integrations) using the matching condition (6.12), we arrive at (6.16).

\subsection{Critical layers and their continuity properties}

In this section we present the proof of Lemma 3.5. We first rewrite Eq. (3.2) for $u_{n}$ in the form

$$
u_{n}^{\prime \prime}(r)+\mathcal{P}(r) u_{n}^{\prime}(r)+\mathcal{Q}_{n}(r) u_{n}(r)=0,
$$

\footnotetext{
${ }^{4}$ Except for an ambiguous sentence asserting, without any justification, that the eigenfunctions corresponding to purely imaginary eigenvalues should form a complete family.
} 
where $\mathcal{P}(r)=\mathcal{A}^{\prime}(r) / \mathcal{A}(r)+1 / r$ and

$$
\mathcal{Q}_{n}(r)=\frac{k^{2}}{m^{2}} \frac{\Phi_{n}(r)}{\gamma_{n}(r)^{2}}-\frac{r}{\mathcal{A}(r) \gamma_{n}(r)} \partial_{r}\left(\frac{W_{n}(r)}{m^{2}+k^{2} r^{2}}\right)+\frac{\mathcal{P}(r)}{r}-\frac{2}{r^{2}} .
$$

Here, as in Section 4.3, we denote $\gamma_{n}(r)=\Omega_{n}(r)-b_{n}-i a_{n}$, where $\Omega_{n}$ is the angular velocity associated with $W_{n}$ as in (1.16). By assumption $\left.i i\right)$ and (1.16) we have $\Omega_{n} \rightarrow \Omega$ in $\mathcal{C}^{2}$ on compact subsets of $(0, \infty)$. In view of $i i i), \Omega$ is analytic in $\mathbb{D}(\bar{r}, \rho)$ for some $\rho>0$, and $\Omega_{n}$ converges uniformly to $\Omega$ on that disc as $n \rightarrow \infty$. Since $\Omega(\bar{r})=b$ and $\Omega^{\prime}(\bar{r})<0$, it follows from Hurwitz's theorem that, for sufficiently large $n$, there exists a unique $\bar{r}_{n} \in \mathbb{D}(\bar{r}, \rho)$ such that $\Omega_{n}\left(\bar{r}_{n}\right)=b_{n}+i a_{n}$. Moreover $\Omega_{n}^{\prime}\left(\bar{r}_{n}\right) \neq 0, \Omega_{n}^{\prime}(\bar{r})<0$ and

$$
\bar{r}_{n}=\bar{r}+\frac{b_{n}+i a_{n}-\Omega_{n}(\bar{r})}{\Omega_{n}^{\prime}(\bar{r})}+\mathcal{O}\left(\left|b_{n}-\Omega_{n}(\bar{r})\right|^{2}+a_{n}^{2}\right), \quad \text { as } n \rightarrow \infty,
$$

so that $\bar{r}_{n} \rightarrow \bar{r}$ and $\bar{r}_{n} \in\{z \in \mathbb{C} ; \operatorname{Im}(z)<0\}$ when $n$ is sufficiently large. By construction, we also have $\gamma_{n}(r)=\Omega_{n}^{\prime}\left(\bar{r}_{n}\right)\left(r-\bar{r}_{n}\right)+\mathcal{O}\left(\left|r-\bar{r}_{n}\right|^{2}\right)$ as $r \rightarrow \bar{r}_{n}$.

Multiplying (6.18) by $z^{2}$ and applying the change of variables $z=r-\bar{r}_{n}, w_{n}(z)=u_{n}\left(\bar{r}_{n}+z\right)$, we obtain the canonical form

$$
z^{2} w_{n}^{\prime \prime}(z)+z P_{n}(z) w_{n}^{\prime}(z)+Q_{n}(z) w_{n}(z)=0,
$$

where $P_{n}(z)=z \mathcal{P}\left(\bar{r}_{n}+z\right)$ and $Q_{n}(z)=z^{2} \mathcal{Q}_{n}\left(\bar{r}_{n}+z\right)$ are analytic inside the disc $\mathbb{D}(0, \rho / 2)$, if $n$ is large enough so that $\left|\bar{r}_{n}-\bar{r}\right|<\rho / 2$. In this situation, the Frobenius method [6, Section 4.8], which we briefly recall now, can be used to construct solutions of (6.19) in $\mathbb{D}(0, \rho / 2) \backslash \mathbb{R}_{\text {_ of }}$ the form

$$
w_{n}(z)=z^{d_{n}} v_{n}(z), \quad \text { where } v_{n}(z)=\sum_{k=0}^{\infty} c_{n, k} z^{k} \text { and } c_{n, 0}=1 \text { for all } n .
$$

The coefficients $c_{n, k}$ for $k \geq 1$ are determined by substituting (6.20) into (6.19) and collecting equal powers of $z$. If $P_{n}(z)=\sum_{k=0}^{\infty} p_{n, k} z^{k}$ and $Q_{n}(z)=\sum_{k=0}^{\infty} q_{n, k} z^{k}$, one obtains the recursion relations

$$
c_{n, k}=\frac{-1}{f_{n}\left(d_{n}+k\right)} \sum_{j=0}^{k-1} c_{n, j}\left[\left(d_{n}+j\right) p_{n, k-j}+q_{n, k-j}\right], \quad k \geq 1,
$$

where the indicial function $f_{n}: \mathbb{R} \rightarrow \mathbb{R}$ is defined by

$$
f_{n}(d)=d(d-1)+d p_{n, 0}+q_{n, 0} \cdot
$$

Assuming that $f_{n}\left(d_{n}+k\right) \neq 0$ for all $k \geq 1$, it is straightforward to verify that the formal series $w_{n}(z)$ defined by (6.20), (6.21) satisfies

$$
z^{2} w_{n}^{\prime \prime}(z)+z P_{n}(z) w_{n}^{\prime}(z)+Q_{n}(z) w_{n}(z)=f_{n}\left(d_{n}\right) z^{d_{n}},
$$

hence is a (formal) solution of (6.19) provided $d_{n}$ is a root of the quadratic polynomial $f_{n}$.

In our situation we have $p_{n, 0}=0$ and $q_{n, 0}=\left(k^{2} / m^{2}\right) J_{n}\left(\bar{r}_{n}\right)$, so that the indicial equation $f_{n}\left(d_{n}\right)=0$ reduces to Eq. (3.19) as $n \rightarrow \infty$. The roots $d_{n}^{ \pm}$of $f_{n}$ thus converge to the explicit values $d_{ \pm}$described in Section 3.4, which are such that $\left|d_{+}-d_{-}\right|<1$. As a consequence, if $n$ is large enough and $d_{n}=d_{n}^{+}$or $d_{n}^{-}$, the denominator in (6.21) never vanishes, and even satisfies $\left|f_{n}\left(d_{n}+k\right)\right| \geq c_{0} k^{2}$ for all $k \geq 1$, where $c_{0}>0$ is independent of $n$. This allows us to solve the recursion relations (6.21) if $d_{n}=d_{n}^{ \pm}$, and it is then straightforward to verify that the series in (6.20) converges for all $z \in \mathbb{D}(0, \rho / 2)$, and that the sum $v_{n}^{ \pm}$is uniformly bounded on compact 
subsets of $\mathbb{D}(0, \rho / 2)$ if $n$ is sufficiently large. We denote henceforth by $w_{n}^{ \pm}(z)=z^{d_{n}^{ \pm}} v_{n}^{ \pm}(z)$ the solution of (6.19) given by (6.20) with $d_{n}=d_{n}^{ \pm}$.

By assumption iii), the quantities $p_{n, k}, q_{n, k}$ converge as $n \rightarrow \infty$ to the Taylor coefficients of the functions $P(z), Q(z)$ associated with the limiting profile $W$ and the limiting value of the spectral parameter. Using the recursion relation (6.21), where each coefficient $c_{n, k}^{ \pm}$is entirely determined by a finite number of coefficients $p_{n, \ell}, q_{n, \ell}$ (namely, those with $\ell<k$ ), we see that

$$
c_{n, k}^{ \pm} \rightarrow c_{k}^{ \pm} \text {as } n \rightarrow \infty
$$

where $c_{k}^{ \pm}$denote the coefficients of the Frobenius solution $w^{ \pm}(z)=z^{d_{ \pm}} v^{ \pm}(z)$ of the limiting equation (6.19), where $P_{n}, Q_{n}$ are replaced by $P, Q$. In view of the uniform bounds mentioned above, this implies that $v_{n}^{ \pm}(z)$ converges to $v^{ \pm}(z)$ uniformly on compact subsets of $\mathbb{D}(0, \rho / 2)$ as $n \rightarrow \infty$. Note that since $P$ and $Q$ are real valued on the real axis, the recurrence relation yields that the coefficients $c_{k}^{ \pm}$are real too. The functions $V_{ \pm}$which appear in the formulas (3.20) - (3.22) are the only real analytic functions on $\mathbb{R}_{+}$that satisfy

$$
V_{ \pm}(r)=v^{ \pm}(r-\bar{r})\left(\frac{r-\bar{r}}{b-\Omega(r)}\right)^{d_{ \pm}}, \quad r \in(\bar{r}-\rho / 2, \bar{r}+\rho / 2) .
$$

That $V_{ \pm}$are well defined and real analytic on the whole half-line $\mathbb{R}_{+}$follows from the representation (3.20) and the ODE (3.18).

Now, consider a sequence $\left(u_{n}\right)_{n \in \mathbb{N}}$ of solutions of (6.18) as in the statement of Lemma 3.5, and assume first that $d_{+} \neq d_{-}$, so that $d_{n}^{+} \neq d_{n}^{-}$when $n$ is sufficiently large. Since (6.18) is a second order differential equation, there exist complex coefficients $\alpha_{n}^{ \pm}$such that 5

$$
u_{n}(r)=\alpha_{n}^{+} w_{n}^{+}\left(r-\bar{r}_{n}\right)+\alpha_{n}^{-} w_{n}^{-}\left(r-\bar{r}_{n}\right), \quad \text { for } r \in(\bar{r}-\rho / 4, \bar{r}+\rho / 4) .
$$

By assumption $u_{n}(r)$ and $u_{n}^{\prime}(r)$ have a limit as $n \rightarrow \infty$ for some $r \neq \bar{r}$, and using elementary continuity properties for solutions of nonsingular ODEs we deduce that convergence holds locally uniformly for all $r>\bar{r}$, or for all $r<\bar{r}$. Since the functions $w_{n}^{ \pm}\left(r-\bar{r}_{n}\right)$ converge uniformly to $w^{ \pm}(r-\bar{r})$ in a neighborhood of $\bar{r}$, and since the limits $w^{ \pm}(r-\bar{r})$ have genuinely different behaviors as $r \rightarrow \bar{r}$, this implies that the coefficients $\alpha_{n}^{ \pm}$in (6.25) have finite limits $\alpha_{ \pm} \in \mathbb{C}$ as $n \rightarrow \infty$. In particular we have

$$
u_{n}(r) \rightarrow \alpha_{+}(r-\bar{r})^{d_{+}} v^{+}(r-\bar{r})+\alpha_{-}(r-\bar{r})^{d_{-}} v^{-}(r-\bar{r}),
$$

uniformly for $r \in(\bar{r}, \bar{r}+\rho / 4)$, and (keeping in mind that $\operatorname{Im}\left(\bar{r}_{n}\right)<0$ )

$$
u_{n}(r) \rightarrow \alpha_{+} e^{i \pi d_{+}}(\bar{r}-r)^{d_{+}} v^{+}(r-\bar{r})+\alpha_{-} e^{i \pi d_{-}}(\bar{r}-r)^{d_{-}} v^{-}(r-\bar{r}),
$$

uniformly for $r \in(\bar{r}-\rho / 4, \bar{r})$. Since outside the interval $(\bar{r}-\rho / 4, \bar{r}+\rho / 4)$ the ODE (6.18) is asymptotically regular, this implies the desired conclusion, namely that $u_{n} \rightarrow \alpha_{+} \phi_{+}+\alpha_{-} \phi_{-}$ where $\phi_{ \pm}$are as in (3.20) or (3.21).

We next consider the exceptional situation where $d_{-}=d_{+}$. Without loss of generality we may assume that either $d_{n}^{-}=d_{n}^{+}$for all $n \in \mathbb{N}$, or $d_{n}^{-} \neq d_{n}^{+}$for all $n \in \mathbb{N}$. In the first case we obtain from (6.20) only one solution $w_{n}$ of (6.19), but we can construct a second solution by differentiating (6.23) with respect to the exponent $d_{n}$, taking into account the fact that $f_{n}^{\prime}\left(d_{n}\right)=0$ since $d_{n}=d_{n}^{ \pm}$is a double root by assumption. The new solution has the form

$$
w_{n}^{\sharp}(z)=\log (z) w_{n}(z)+z^{d_{n}} \sum_{k=0}^{\infty}\left(\frac{\partial c_{n, k}}{\partial d_{n}}\right) z^{k}, \quad z \in \mathbb{D}(0, \rho / 2) \backslash \mathbb{R}_{-},
$$

\footnotetext{
${ }^{5}$ Note that $\mathbb{D}\left(\bar{r}_{n}, \rho / 2\right)$ contains the real interval $(\bar{r}-\rho / 4, \bar{r}+\rho / 4)$ for large values of $n$.
} 
and its asymptotic behavior as $z \rightarrow 0$ is clearly different from that of $w_{n}(z)$. This allows us to conclude the proof using the same argument as above, and we obtain that $u_{n} \rightarrow \alpha_{+} \phi_{+}+\alpha_{-} \phi_{-}$ where $\phi_{ \pm}$are as in (3.22). On the other hand, when $d_{n}^{-} \neq d_{n}^{+}$for all $n \in \mathbb{N}$ (but $d_{n}^{-}-d_{n}^{+} \rightarrow 0$ ), the decomposition (6.25) is not appropriate, because in that case we cannot prove that the coefficients $\alpha_{n}^{+}$and $\alpha_{n}^{-}$are bounded, yet alone have limits as $n \rightarrow \infty$. Instead, we write

$$
u_{n}(r)=\alpha_{n}\left(w_{n}^{+}\left(r-\bar{r}_{n}\right)+w_{n}^{-}\left(r-\bar{r}_{n}\right)\right)+\alpha_{n}^{\sharp}\left(\frac{w_{n}^{+}\left(r-\bar{r}_{n}\right)-w_{n}^{-}\left(r-\bar{r}_{n}\right)}{d_{n}^{+}-d_{n}^{-}}\right),
$$

for $r \in(\bar{r}-\rho / 4, \bar{r}+\rho / 4)$, and this new decomposition has the property that both coefficients $\alpha_{n}$ and $\alpha_{n}^{\sharp}$ necessarily have limits as $n \rightarrow \infty$. We then finish the proof along the same lines as above.

\subsection{Approximation and interpolation in the class $\mathscr{W}$}

This section is devoted to the proof of Lemmas 4.2 and 4.3 , If $W$ is a vorticity profile that belongs to the class $\mathscr{W}$, in the sense of Definition 1.8 , we denote by $\Omega$ the corresponding angular velocity given by (1.16), and by $J$ the function defined in (1.20). The first observation is that both $\Omega$ and $W$ can be expressed in terms of the auxiliary function $J$.

Indeed, let $\phi: \mathbb{R}_{+} \rightarrow \mathbb{R}$ be defined by $\phi(r)=\Omega(r) / \Omega^{\prime}(r)$ for all $r>0$. According to (1.6), (1.19), (1.20) we have

$$
J(r)=\frac{\Phi(r)}{\Omega^{\prime}(r)^{2}}=\frac{2 r \Omega(r)}{\Omega^{\prime}(r)}+\frac{4 \Omega(r)^{2}}{\Omega^{\prime}(r)^{2}}=2 r \phi(r)+4 \phi(r)^{2},
$$

for all $r>0$. Since $J(r)>0$ and $\phi(r)<0$ by assumption, we deduce that

$$
\phi(r)=\frac{\Omega(r)}{\Omega^{\prime}(r)}=-\frac{1}{4}\left(r+\sqrt{r^{2}+4 J(r)}\right), \quad r>0 .
$$

Integrating this differential equation and using the normalization condition $\Omega(0)=1$, which follows from (1.16) since $W(0)=2$, we obtain the representation formula

$$
\Omega(r)=\exp \left(-\int_{0}^{r} \frac{4}{s+\sqrt{s^{2}+4 J(s)}} \mathrm{d} s\right), \quad r>0 .
$$

As $W(r)=r \Omega^{\prime}(r)+2 \Omega(r)$, we also have

$$
W(r)=\Omega(r)\left(2+\frac{r}{\phi(r)}\right)=\Omega(r)\left(2-\frac{4 r}{r+\sqrt{r^{2}+4 J(r)}}\right), \quad r>0 .
$$

Furthermore, if we differentiate (6.30) with respect to $r$ and observe that $\Omega^{\prime}=\Omega / \phi$, we obtain

$$
W^{\prime}=\frac{\Omega}{\phi}\left(2+\frac{r}{\phi}\right)+\Omega\left(\frac{1}{\phi}-\frac{r \phi^{\prime}}{\phi^{2}}\right)=\frac{\Omega}{\phi^{2}}\left(3 \phi+r-r \phi^{\prime}\right) .
$$

Thus, using the expression (6.28) of $\phi$, we find after elementary calculations

$$
W^{\prime}(r)=\frac{8 \Omega(r)}{\left(r+\sqrt{r^{2}+4 J(r)}\right)^{2}}\left(r-\frac{r^{2}+6 J(r)}{\sqrt{r^{2}+4 J(r)}}+\frac{r J^{\prime}(r)}{\sqrt{r^{2}+4 J(r)}}\right) .
$$

As $r \sqrt{r^{2}+4 J}<r^{2}+6 J$ when $J>0$, this formula shows that the vorticity $W$ is strictly decreasing as soon as the auxiliary function $J$ satisfies $J(r)>0$ and $J^{\prime}(r)<0$ for all $r>0$. This observation will be used later on. 
Since $W \in \mathscr{W}$ by assumption, the angular velocity satisfies $\Omega^{\prime}(r) \rightarrow 0$ as $r \rightarrow 0$, and in view of (1.20) or (6.28) this implies that $J(r) \rightarrow \infty$ as $r \rightarrow 0$. It then follows from (6.31) that

$$
W^{\prime}(r) \sim \frac{r J^{\prime}(r)}{J(r)^{3 / 2}}-\frac{6}{J(r)^{1 / 2}}, \quad \text { as } \quad r \rightarrow 0,
$$

and since $W^{\prime}(r)$ vanishes at the origin we deduce that $r J^{\prime}(r) J(r)^{-3 / 2} \rightarrow 0$ as $r \rightarrow 0$. Concerning the behavior at infinity, we observe that $\phi(r)=-2 / r+\mathcal{O}\left(1 / r^{3}\right)$ as $r \rightarrow \infty$, and in view of (6.29) this implies that $\Omega(r)=\Gamma / r^{2}+\mathcal{O}\left(1 / r^{4}\right)$ as $r \rightarrow \infty$, for some $\Gamma>0$. The expression (6.30) also shows that $r^{4} W(r) \rightarrow 2 \Gamma J(\infty)$ as $r \rightarrow \infty$. Finally, one infers from (6.31) that

$$
W^{\prime}(r) \sim\left\{\frac{2 \Gamma}{r^{4}}+\mathcal{O}\left(\frac{1}{r^{6}}\right)\right\}\left\{\frac{r J^{\prime}(r)}{\sqrt{r^{2}+4 J(r)}}-\frac{4 J(r)}{r}+\mathcal{O}\left(\frac{1}{r^{3}}\right)\right\}, \quad \text { as } \quad r \rightarrow \infty,
$$

and since $r J^{\prime}(r) \rightarrow 0$ as $r \rightarrow \infty$ we also obtain $r^{5} W^{\prime}(r) \rightarrow-8 \Gamma J(\infty)$ as $r \rightarrow \infty$.

The properties of $J$ are more conveniently expressed in terms of the new function

$$
Q(r)=\frac{1}{\sqrt{1+J(r)}}, \quad r>0 .
$$

Definition 6.3. We say that a $\mathcal{C}^{1}$ function $Q: \mathbb{R}_{+} \rightarrow(0,1]$ belongs to the class $\mathscr{Q}$ if

i) $Q^{\prime}(r)>0$ for all $r>0$;

ii) $Q(r) \rightarrow 0$ and $r Q^{\prime}(r) \rightarrow 0$ as $r \rightarrow 0$;

iii) $r Q^{\prime}(r) \rightarrow 0$ as $r \rightarrow \infty$.

In particular $\mathscr{Q}$ is convex, and if $Q \in \mathscr{Q}$ then $\mathcal{N}(Q):=\sup _{r>0} r\left|Q^{\prime}(r)\right|<\infty$.

Lemma 6.4. A vorticity profile $W$ belongs to the class $\mathscr{W}$ in the sense of Definition 1.8 if and only if the function $Q$ defined by (6.32), with $J$ as in (1.20), belongs to the class $\mathscr{Q}$.

Proof. If $W \in \mathscr{W}$, we have just shown that the $\mathcal{C}^{1}$ map $J: \mathbb{R}_{+} \rightarrow \mathbb{R}$ defined by (1.20) satisfies $J(r)>0$ and $J^{\prime}(r)<0$ for all $r>0, J(r) \rightarrow \infty$ and $r J^{\prime}(r) J(r)^{-3 / 2} \rightarrow 0$ as $r \rightarrow 0$, and $r J^{\prime}(r) \rightarrow 0$ as $r \rightarrow \infty$. These properties precisely mean that the function $Q$ defined by (6.32) belongs to the class $\mathscr{Q}$. Conversely, if $Q \in \mathscr{Q}$, we define $J=Q^{-2}-1$ so that (6.32) holds, and we reconstruct the angular velocity $\Omega$ and the vorticity $W$ by the formulas (6.29), (6.30). The calculations above then show that $W \in \mathscr{W}$. In particular, since $J^{\prime}(r)<0$, formula (6.31) shows that $W^{\prime}(r)<0$ for all $r>0$.

The following result expresses the fact that the vorticity profile $W \in \mathscr{W}$ depends continuously on the auxiliary function $Q \in \mathscr{Q}$, in appropriate topologies.

Lemma 6.5. Assume that $Q_{1}, Q_{2} \in \mathscr{Q}$, and take $\delta>0$ small enough so that

$$
\delta \leq \min \left\{Q_{1}(1), Q_{2}(1)\right\} \leq \max \left\{Q_{1}(1), Q_{2}(1)\right\} \leq \sqrt{1-\delta^{2}} .
$$

Then there exists a constant $C>0$, depending only on $\delta$, such that, if $W_{1}, W_{2}$ denote the vorticity profiles associated with $Q_{1}, Q_{2}$ as in Lemma 6.4, the following estimates hold:

$$
\begin{aligned}
& \sup _{r>0}\left(1+r^{4}\right)\left|W_{1}(r)-W_{2}(r)\right| \leq C\left\|Q_{1}-Q_{2}\right\|_{L^{\infty}\left(\mathbb{R}_{+}\right)}, \\
& \sup _{r>0}\left(1+r^{5}\right)\left|W_{1}^{\prime}(r)-W_{2}^{\prime}(r)\right| \leq C \mathcal{N}\left(Q_{1}-Q_{2}\right)+C\left(1+\mathcal{N}\left(Q_{2}\right)\right)\left\|Q_{1}-Q_{2}\right\|_{L^{\infty}\left(\mathbb{R}_{+}\right)} .
\end{aligned}
$$


Proof. Let $J_{i}(r)=Q_{i}(r)^{-2}-1$ for $i=1,2$. We first consider the quantity

$$
\Theta(r)=\frac{1}{r+\sqrt{r^{2}+4 J_{1}}}-\frac{1}{r+\sqrt{r^{2}+4 J_{2}}}=\frac{4\left(Q_{1}^{2}-Q_{2}^{2}\right)}{\left(Q_{1} \Delta_{2}+Q_{2} \Delta_{1}\right)\left(r Q_{1}+\Delta_{1}\right)\left(r Q_{2}+\Delta_{2}\right)},
$$

where we use the shorthand notation $\Delta_{i}(r)=Q_{i}(r) \sqrt{r^{2}+4 J_{i}(r)}=\sqrt{4+\left(r^{2}-4\right) Q_{i}(r)^{2}}$ for $i=1,2$. We claim that

$$
\sup _{r>0}\left(1+r^{3}\right)|\Theta(r)| \leq C\left\|Q_{1}-Q_{2}\right\|_{L^{\infty}\left(\mathbb{R}_{+}\right)},
$$

for some constant $C>0$ depending only on $\delta$. To prove that, we distinguish two cases:

i) In the region where $r \leq 1$, we know from (6.33) that $Q_{i}(r)^{2} \leq Q_{i}(1)^{2} \leq 1-\delta^{2}$, and this implies that $\Delta_{i}(r) \geq 2 \sqrt{1-Q_{i}(r)^{2}} \geq 2 \delta$. It follows that

$$
|\Theta(r)| \leq \frac{4\left(Q_{1}+Q_{2}\right)\left|Q_{1}-Q_{2}\right|}{8 \delta^{3}\left(Q_{1}+Q_{2}\right)} \leq \frac{\left|Q_{1}-Q_{2}\right|}{2 \delta^{3}}, \quad 0<r \leq 1 .
$$

ii) When $r \geq 1$ we observe that $\Delta_{i}(r) \geq r Q_{i} \geq r \delta$ by (6.33), so that

$$
|\Theta(r)| \leq \frac{4\left(Q_{1}+Q_{2}\right)\left|Q_{1}-Q_{2}\right|}{8 r^{3} Q_{1}^{2} Q_{2}^{2}} \leq \frac{\left|Q_{1}-Q_{2}\right|}{\delta^{3} r^{3}}, \quad r \geq 1 .
$$

Altogether, this proves (6.36).

As an immediate consequence, we see that the angular velocities defined by (6.29) satisfy the estimate $\left\|\Omega_{1}-\Omega_{2}\right\|_{L^{\infty}\left(\mathbb{R}_{+}\right)} \leq C\left\|Q_{1}-Q_{2}\right\|_{L^{\infty}\left(\mathbb{R}_{+}\right)}$. In fact, we have a stronger result:

$$
\sup _{r>0}\left(1+r^{2}\right)\left|\Omega_{1}(r)-\Omega_{2}(r)\right| \leq C\left\|Q_{1}-Q_{2}\right\|_{L^{\infty}\left(\mathbb{R}_{+}\right)} .
$$

Indeed, it follows from (6.29) that

$$
r^{2} \Omega_{i}(r)=\Omega_{i}(1) \exp \int_{1}^{r}\left(\frac{2}{s}-\frac{4}{s+\sqrt{s^{2}+4 J_{i}(s)}}\right) \mathrm{d} s, \quad r>0, \quad i=1,2 .
$$

If we denote

$$
M_{i}(r)=\frac{1}{r}-\frac{2}{r+\sqrt{r^{2}+4 J_{i}}}=\frac{4 J_{i}}{r\left(r+\sqrt{r^{2}+4 J_{i}}\right)^{2}}=\frac{4\left(1-Q_{i}^{2}\right)}{r\left(r Q_{i}+\Delta_{i}\right)^{2}}>0, \quad r>0,
$$

the same estimates as above show that

$$
\sup _{0<r \leq 1} r M_{i}(r) \leq \frac{1}{\delta^{2}}, \quad \text { and } \quad \sup _{r \geq 1} r^{3} M_{i}(r) \leq \frac{1}{\delta^{2}} .
$$

This implies in particular that $r^{2} \Omega_{i}(r) \leq e^{1 / \delta^{2}}$ for $r \geq 1$, hence $\left(1+r^{2}\right) \Omega_{i}(r) \leq C$ for some constant $C>0$ depending only on $\delta$. In addition, using (6.36) and (6.38), we obtain

$$
r^{2}\left|\Omega_{1}(r)-\Omega_{2}(r)\right| \leq e^{1 / \delta^{2}}\left(\left|\Omega_{1}(1)-\Omega_{2}(1)\right|+4 \int_{1}^{r}|\Theta(s)| \mathrm{d} s\right) \leq C\left\|Q_{1}-Q_{2}\right\|_{L^{\infty}\left(\mathbb{R}_{+}\right)},
$$

for all $r \geq 1$, and this concludes the proof of 6.37).

On the other hand, in view of (6.30), we have $W_{i}(r)=2 r \Omega_{i}(r) M_{i}(r)$ for $i=1,2$, hence

$$
W_{1}(r)-W_{2}(r)=2 r\left(\Omega_{1}(r)-\Omega_{2}(r)\right) M_{1}(r)-4 r \Omega_{2}(r) \Theta(r), \quad r>0 .
$$


Thus using estimates (6.36), (6.37), (6.39) we arrive at (6.34).

Finally, we deduce from (6.31) that $W_{1}^{\prime}(r)-W_{2}^{\prime}(r)=\Xi(r)-16 \Lambda(r)$, where $\Xi(r)$ collects all terms that do not involve the derivatives $J_{1}^{\prime}, J_{2}^{\prime}$, and

$$
\Lambda(r)=\frac{\Omega_{1} r Q_{1}^{\prime}}{\left(r Q_{1}+\Delta_{1}\right)^{2} \Delta_{1}}-\frac{\Omega_{2} r Q_{2}^{\prime}}{\left(r Q_{2}+\Delta_{2}\right)^{2} \Delta_{2}}=\Lambda_{1}(r)+\Lambda_{2}(r),
$$

where

$$
\Lambda_{1}(r)=\frac{\Omega_{1} r\left(Q_{1}^{\prime}-Q_{2}^{\prime}\right)}{\left(r Q_{1}+\Delta_{1}\right)^{2} \Delta_{1}}, \quad \Lambda_{2}(r)=\frac{\Omega_{1} r Q_{2}^{\prime}}{\left(r Q_{1}+\Delta_{1}\right)^{2} \Delta_{1}}-\frac{\Omega_{2} r Q_{2}^{\prime}}{\left(r Q_{2}+\Delta_{2}\right)^{2} \Delta_{2}} .
$$

Proceeding exactly as above it is straightforward to verify that

$$
\sup _{r>0}\left(1+r^{5}\right)|\Xi(r)| \leq C\left\|Q_{1}-Q_{2}\right\|_{L^{\infty}\left(\mathbb{R}_{+}\right)},
$$

where $C>0$ depends only on $\delta$. We thus concentrate on the new term $\Lambda$, which contains the derivatives $Q_{1}^{\prime}, Q_{2}^{\prime}$. Again, considering separately the regions where $r \leq 1$ and $r \geq 1$, and using the appropriate lower bound on $\Delta_{1}(r)$ in each region, we obtain

$$
\left|\Lambda_{1}(r)\right| \leq C \Omega_{1}(r) \frac{r\left|Q_{1}^{\prime}(r)-Q_{2}^{\prime}(r)\right|}{1+r^{3}} \leq C\left(1+r^{2}\right) \Omega_{1}(r) \frac{\mathcal{N}\left(Q_{1}-Q_{2}\right)}{1+r^{5}},
$$

where we recall that $(1+r)^{2} \Omega_{1}(r) \leq C$ for some $C>0$ depending only on $\delta$. The quantity $\Lambda_{2}$ is estimated along the same lines:

$$
\left|\Lambda_{2}(r)\right| \leq C\left(1+r^{2}\right)\left|\Omega_{1}(r)-\Omega_{2}(r)\right| \frac{r\left|Q_{2}^{\prime}(r)\right|}{1+r^{5}}+C\left(1+r^{2}\right) \Omega_{2}(r) \frac{r\left|Q_{2}^{\prime}(r)\right|}{1+r^{5}}\left|Q_{1}(r)-Q_{2}(r)\right|,
$$

and using (6.37) we find $\left(1+r^{5}\right)\left|\Lambda_{2}(r)\right| \leq C\left(1+\mathcal{N}\left(Q_{2}\right)\right)\left\|Q_{1}-Q_{2}\right\|_{L^{\infty}\left(\mathbb{R}_{+}\right)}$. Combining these estimates we arrive at (6.35).

Proof of Lemma 4.2. Let $W \in \mathscr{W}$, and denote by $Q \in \mathscr{Q}$ the function defined by (6.32) with $J$ as in (1.20). For any $\epsilon>0$ we define

$$
Q^{(\epsilon)}(r)=\frac{1}{\sqrt{\pi \epsilon}} \int_{0}^{\infty}\left(e^{-(r-s)^{2} / \epsilon}-e^{-(r+s)^{2} / \epsilon}\right) Q(s) \mathrm{d} s, \quad r>0 .
$$

In other words, $Q^{(\epsilon)}$ is the restriction to $\mathbb{R}_{+}$of the real-analytic odd function obtained by extending $Q$ to an odd function $\bar{Q}: \mathbb{R} \rightarrow \mathbb{R}$ and applying to $\bar{Q}$ the heat semigroup on $\mathbb{R}$ at time $t=\epsilon / 4$. In particular, $Q^{(\epsilon)}$ is real-analytic on $\overline{\mathbb{R}}_{+}$for any $\epsilon>0$. Moreover, as the function $s \mapsto \bar{Q}(\mathrm{~s})$ is continuous on $\mathbb{R}$ and converges to finite limits as $s \rightarrow \pm \infty$, it is clear that $Q^{(\epsilon)}$ converges uniformly to $Q$ on $\mathbb{R}_{+}$as $\epsilon \rightarrow 0$. On the other hand, differentiating (6.40) with respect to $r$, we obtain the relation

$$
r Q^{(\epsilon) \prime}(r)=\frac{1}{\sqrt{\pi \epsilon}} \int_{0}^{\infty}\left(e^{-(r-s)^{2} / \epsilon}-e^{-(r+s)^{2} / \epsilon}\right) s Q^{\prime}(s) \mathrm{d} s+R_{\epsilon}(r), \quad r>0
$$

where

$$
R_{\epsilon}(r)=\frac{1}{\sqrt{\pi \epsilon}} \int_{0}^{\infty}\left((r-s) e^{-(r-s)^{2} / \epsilon}+(r+s) e^{-(r+s)^{2} / \epsilon}\right) Q^{\prime}(s) \mathrm{d} s, \quad r>0 .
$$


As $Q^{\prime} \in L^{1}\left(\mathbb{R}_{+}\right)$and $\mathcal{N}(Q)<\infty$, it is straightforward to verify that $R_{\epsilon}$ converges uniformly to zero as $\epsilon \rightarrow 0$. Moreover, since $s Q^{\prime}(s) \rightarrow 0$ as $s \rightarrow 0$ and $s \rightarrow \infty$, it is clear that the integral term in (6.41) converges uniformly on $\mathbb{R}_{+}$towards $r Q^{\prime}(r)$ as $\epsilon \rightarrow 0$. Altogether, this shows that

$$
\lim _{\epsilon \rightarrow 0}\left(\left\|Q^{(\epsilon)}-Q\right\|_{L^{\infty}\left(\mathbb{R}_{+}\right)}+\left|\mathcal{N}\left(Q^{(\epsilon)}-Q\right)\right|\right)=0 .
$$

Now, let $W^{(\epsilon)} \in \mathscr{W}$ be the vorticity profile associated with $Q^{(\epsilon)}$ as in Lemma 6.4. By construction $W^{(\epsilon)}$ is real-analytic on $\overline{\mathbb{R}}_{+}$for any $\epsilon>0$, and it follows from (6.42) and Lemma 6.5 that $W^{(\epsilon)} \rightarrow W$ in the topology of $\mathcal{C}_{b}^{1}\left(\overline{\mathbb{R}}_{+}\right)$as $\epsilon \rightarrow 0$. This is the desired density result.

Proof of Lemma 4.3. Assume that $W_{0}, W_{1} \in \mathscr{W}$, and let $Q_{1}, Q_{2} \in \mathscr{Q}$ be the corresponding functions defined as in Lemma 6.4. For any $t \in[0,1]$, we define $Q_{t} \in \mathscr{Q}$ by the linear interpolation formula

$$
Q_{t}(r)=(1-t) Q_{0}(r)+t Q_{1}(r), \quad r>0,
$$

and we denote $W_{t} \in \mathscr{W}$ the vorticity profile associated with $Q_{t}$. As

$$
\left\|Q_{t_{1}}-Q_{t_{2}}\right\|_{L^{\infty}\left(\mathbb{R}_{+}\right)}+\mathcal{N}\left(Q_{t_{1}}-Q_{t_{2}}\right)=\left|t_{1}-t_{2}\right|\left(\left\|Q_{0}-Q_{1}\right\|_{L^{\infty}\left(\mathbb{R}_{+}\right)}+\mathcal{N}\left(Q_{0}-Q_{1}\right)\right),
$$

it follows from Lemma 6.5 that $W_{t}$ is a Lipschitz function of $t \in[0,1]$ in the topology of $\mathcal{C}_{b}^{1}\left(\overline{\mathbb{R}}_{+}\right)$. By construction, if $W_{0}, W_{1}$ are real analytic on $\overline{\mathbb{R}}_{+}$, so is $W_{t}$ for any $t \in[0,1]$. Moreover, in that case, if $W_{1}^{\prime \prime}(0) \equiv-8 Q_{1}^{\prime}(0)<0$, then $W_{t}^{\prime \prime}(0) \equiv-8 Q_{t}^{\prime}(0)<0$ for all $t \in(0,1]$, because $W_{0}^{\prime \prime}(0) \equiv-8 Q_{0}^{\prime}(0) \leq 0$. This concludes the proof.

\subsection{Proof of Lemma 4.6.}

The proof of estimate (4.32) is lengthy but rather straightforward, and we just indicate here the main steps. Using classical estimates for the modified Bessel functions $K_{\nu}$ [1 , Section 9.6], we first observe that the approximate solution $\chi_{n}$ defined in (4.26) satisfies

$$
\begin{aligned}
& \left|\chi_{n}(r)\right| \approx \min \left(1,\left|r-r_{n}+i c_{n}\right|\right)^{\frac{1}{2}-\nu_{n}} e^{-k\left(r-r_{n}\right)}, \\
& \left|\chi_{n}^{\prime}(r)\right| \approx \min \left(1,\left|r-r_{n}+i c_{n}\right|\right)^{-\frac{1}{2}-\nu_{n}} e^{-k\left(r-r_{n}\right)},
\end{aligned}
$$

for all $r>0$. Here $A \approx B$ means that the ratio $A / B$ is bounded from above and from below by some positive constants that are independent of $n$. A direct consequence of (6.44) is :

Lemma 6.6. There exists a constant $C>0$ such that, for all $n \in \mathbb{N}$ and all $s>r>0$, the following estimates hold:

$$
\begin{array}{r}
\left|\chi_{n}(s)\right|^{2} \int_{r}^{s} \frac{1}{\left|\chi_{n}(t)\right|^{2}} \mathrm{~d} t \leq C \min \left(1,\left|s-r_{n}+i c_{n}\right|\right)^{1-2 \nu_{n}}, \\
\left|\chi_{n}(s) \chi_{n}^{\prime}(s)\right| \int_{r}^{s} \frac{1}{\left|\chi_{n}(t)\right|^{2}} \mathrm{~d} t \leq C \min \left(1,\left|s-r_{n}+i c_{n}\right|\right)^{-2 \nu_{n}} .
\end{array}
$$

Proof. We only prove (6.45), the proof of (6.46) being similar. If $c_{n} \geq 1$, the first estimate in (6.44) simply means that $\left|\chi_{n}(r)\right| \approx e^{-k\left(r-r_{n}\right)}$, and (6.45) follows immediately. Thus we assume henceforth that $0<c_{n} \leq 1$, and for simplicity we set $s=r_{n}+\tau$, so that the proof of (6.45) reduces to showing that

$$
\mathcal{I}_{n}(\tau):=\left|\chi_{n}\left(r_{n}+\tau\right)\right|^{2} \int_{-\infty}^{\tau} \frac{1}{\left|\chi_{n}\left(r_{n}+t\right)\right|^{2}} \mathrm{~d} t \leq C \min \left(1,\left|\tau+i c_{n}\right|\right)^{1-2 \nu_{n}}, \quad \tau \in \mathbb{R} .
$$


If $\tau \leq-1$, we know from (6.44) that $\left|\chi_{n}\left(r_{n}+\tau\right)\right| \leq C e^{-k \tau}$ and $\left|\chi_{n}\left(r_{n}+t\right)\right| \geq C e^{-k t}$ for $t \leq \tau$, hence $\mathcal{I}_{n}(\tau) \leq C$. If $|\tau| \leq 1$, then $\left.\left|\chi_{n}\left(r_{n}+\tau\right)\right| \leq C\left|\tau+i c_{n}\right|\right)^{\frac{1}{2}-\nu_{n}}$, and

$$
\mathcal{I}_{n}(\tau) \leq C\left|\tau+i c_{n}\right|^{1-2 \nu_{n}}\left(\int_{-\infty}^{-1} e^{2 k t} \mathrm{~d} t+\int_{-1}^{\tau}\left|t+i c_{n}\right|^{2 \nu_{n}-1} \mathrm{~d} t\right) \leq C\left|\tau+i c_{n}\right|^{1-2 \nu_{n}} .
$$

Finally, if $\tau \geq 1$, then

$$
\mathcal{I}_{n}(\tau) \leq C e^{-2 k \tau}\left(\int_{-\infty}^{-1} e^{2 k t} \mathrm{~d} t+\int_{-1}^{1}\left|t+i c_{n}\right|^{2 \nu_{n}-1} \mathrm{~d} t+\int_{1}^{\tau} e^{2 k t} \mathrm{~d} t\right) \leq C,
$$

and this completes the proof of (6.45). We observe that the constant $C$ in the right-hand side is independent of $n$ because, as can be seen from (4.27), the exponent $\nu_{n}$ is bounded away from zero as $n \rightarrow \infty$.

Remark 6.7. In Section 4.9 we use the fact that if $\nu_{n} \rightarrow 0$ as $n \rightarrow \infty$, the conclusion of Lemma 6.6 remains valid up to a logarithmic correction. Estimates (6.44) are not appropriate in that case, but one can use the fact that $K_{\nu_{n}}(z)$ is close to $K_{0}(z)$ for $n$ large, where $K_{0}(z) \approx$ $-\log (z)$ as $z \rightarrow 0$. In particular, the integral

$$
\int_{r_{n}-1}^{r_{n}+1} \frac{1}{\chi_{n}(r)^{2}} \mathrm{~d} r=\frac{\pi}{2} \int_{-1}^{1} \frac{1}{\left(t+i c_{n}\right) K_{\nu_{n}}\left(k\left(t+i c_{n}\right)\right)^{2}} \mathrm{~d} t
$$

is uniformly bounded even if $\nu_{n} \rightarrow 0$ and $c_{n} \rightarrow 0$, because the function $t \mapsto|t|^{-1}\left|K_{0}(k t)\right|^{-2}$ is integrable over $[-1,1]$. We leave the details to the reader.

Returning to the proof of Lemma 4.6, we note that $\mathcal{K}_{n}(r, s)=\mathcal{K}_{n}^{(1)}(r, s)-\mathcal{K}_{n}^{(2)}(r, s)$, where

$$
\begin{aligned}
& \mathcal{K}_{n}^{(1)}(r, s)=\mathcal{A}(s) \chi_{n}(s)^{2} \mathcal{R}_{n}(s) \int_{r}^{s} \frac{1}{\mathcal{A}(t) \chi_{n}(t)^{2}} \mathrm{~d} t \\
& \mathcal{K}_{n}^{(2)}(r, s)=\mathcal{A}^{\prime}(s) \chi_{n}(s) \chi_{n}^{\prime}(s) \int_{r}^{s} \frac{1}{\mathcal{A}(t) \chi_{n}(t)^{2}} \mathrm{~d} t
\end{aligned}
$$

In what follows, we assume that $s \geq r \geq \delta r_{n}$ for some $\delta>0$, where $r_{n}=\left(\Gamma_{n} / b_{n}\right)^{1 / 2} \rightarrow \infty$ as $n \rightarrow \infty$. Since $\mathcal{A}(s) \rightarrow 1 / k^{2}$ and $\mathcal{A}^{\prime}(s)=\mathcal{O}\left(s^{-3}\right)$ as $s \rightarrow \infty$, it follows immediately from (6.46) that

$$
\sup _{r \geq \delta r_{n}} \int_{r}^{\infty}\left|\mathcal{K}_{n}^{(2)}(r, s)\right| \mathrm{d} s \leq C \int_{\delta r_{n}}^{\infty} s^{-3} \min \left(1,\left|s-r_{n}+i c_{n}\right|\right)^{-2 \nu_{n}} \mathrm{~d} s \leq \frac{C}{r_{n}^{2}} \leq C b_{n} .
$$

It thus remains to estimate the kernel $\mathcal{K}_{n}^{(1)}(r, s)$, which involves the remainder $\mathcal{R}_{n}$ defined in (4.29). We can further decompose $\mathcal{R}_{n}=\mathcal{R}_{n}^{(1)}+\mathcal{R}_{n}^{(2)}+\mathcal{R}_{n}^{(3)}$, where

$$
\begin{aligned}
& \mathcal{R}_{n}^{(1)}(r)=\frac{m^{2}+\frac{3}{4}}{r^{2}}-\frac{1}{2 r} \frac{\mathcal{A}^{\prime}(r)}{\mathcal{A}(r)}, \quad \mathcal{R}_{n}^{(2)}(r)=-\frac{k^{2}}{m^{2}}\left(\frac{\Phi_{n}(r)}{\gamma_{n}(r)^{2}}-\frac{J_{n}\left(r_{n}\right)}{\left(r-r_{n}+i c_{n}\right)^{2}}\right), \\
& \mathcal{R}_{n}^{(3)}(r)=\frac{r}{\mathcal{A}(r) \gamma_{n}(r)} \partial_{r}\left(\frac{W_{n}(r)}{m^{2}+k^{2} r^{2}}\right) .
\end{aligned}
$$

We concentrate here on the term $\mathcal{R}_{n}^{(2)}$, which gives the main contribution to the integral (4.32).

Lemma 6.8. For any $\delta>0$, there exists a constant $C>0$ such that

$$
\left|\mathcal{R}_{n}^{(2)}(r)\right| \leq \frac{C}{r\left|r-r_{n}+i c_{n}\right|}, \quad \text { for all } r \geq \delta r_{n} .
$$


Proof. We first assume that $\left|r-r_{n}\right| \leq r_{n} / 2$. Using the same notations as in (4.23), we have

$$
\gamma_{n}(r)=\Omega_{n}(r)-\Omega_{n}\left(r_{n}\right)-i a_{n}=\Omega_{n}^{\prime}\left(r_{n}\right)\left(r-r_{n}+i c_{n}\right)+\left(\Omega^{\prime}(\xi)-\Omega^{\prime}\left(r_{n}\right)\right)\left(r-r_{n}\right),
$$

for some $\xi \in\left[r_{n} / 2,3 r_{n} / 2\right]$. As $\Omega_{n}^{\prime}(s) \approx s^{-3}$ and $\Omega_{n}^{\prime \prime}(s) \approx s^{-4}$ as $s \rightarrow \infty$ (see (4.9) and the first part of Section 6.4), we have

$$
\left|\Omega_{n}^{\prime}(\xi)-\Omega_{n}^{\prime}\left(r_{n}\right)\right| \leq \frac{C}{r_{n}}\left|\Omega_{n}^{\prime}\left(r_{n}\right)\right|\left|\xi-r_{n}\right| \leq \frac{C}{r_{n}}\left|\Omega_{n}^{\prime}\left(r_{n}\right)\right|\left|r-r_{n}\right|,
$$

so that $\gamma_{n}(r)=\Omega_{n}^{\prime}\left(r_{n}\right)\left(r-r_{n}+i c_{n}\right)[1+\mathcal{O}(\epsilon(r))]$, where $\epsilon(r)=\left(r-r_{n}\right) / r_{n}$. Recalling that $J_{n}\left(r_{n}\right)=\Phi_{n}\left(r_{n}\right) / \Omega_{n}^{\prime}\left(r_{n}\right)^{2}$, we obtain the following expression :

$$
\mathcal{R}_{n}^{(2)}(r)=-\frac{k^{2}}{m^{2}} \frac{\Phi_{n}(r)[1+\mathcal{O}(\epsilon(r))]-\Phi_{n}\left(r_{n}\right)}{\Omega^{\prime}\left(r_{n}\right)^{2}\left(r-r_{n}+i c_{n}\right)^{2}} .
$$

Now, it also follows from (4.9) and the first part of Section 6.4 that $\Phi_{n}(s) \approx s^{-6}$ and $\Phi_{n}^{\prime}(s) \approx s^{-7}$ as $s \rightarrow \infty$. This implies that $\left|\Phi_{n}(r)-\Phi_{n}\left(r_{n}\right)\right| \leq C \Phi_{n}\left(r_{n}\right) \epsilon(r)$, and we deduce

$$
\left|\mathcal{R}_{n}^{(2)}(r)\right| \leq C J_{n}\left(r_{n}\right) \frac{\epsilon(r)}{\left|r-r_{n}+i c_{n}\right|^{2}} \leq \frac{C}{r_{n}\left|r-r_{n}+i c_{n}\right|}, \quad \text { if } \quad \frac{\left|r-r_{n}\right|}{r_{n}} \leq \frac{1}{2} .
$$

The argument is simpler if $\left|r_{n}-r\right| \geq r_{n} / 2$, because we can estimate both terms in $\mathcal{R}_{n}^{(2)}$ separately. Straightforward calculations lead to the lower bound

$$
\left|\gamma_{n}(r)\right|=\left|\Omega_{n}(r)-\Omega_{n}\left(r_{n}\right)-i a_{n}\right| \geq C\left|\Omega_{n}^{\prime}(r)\right|\left|r-r_{n}+i c_{n}\right|,
$$

whenever $r \geq \delta r_{n}$ (here $C$ depends on $\delta$ ), and this implies that

$$
\left|\mathcal{R}_{n}^{(2)}(r)\right| \leq C \frac{\Phi_{n}(r)}{\left|\gamma_{n}(r)\right|^{2}}+C \frac{J_{n}\left(r_{n}\right)}{\left|r-r_{n}+i c_{n}\right|^{2}} \leq \frac{C}{\left|r-r_{n}+i c_{n}\right|^{2}}, \quad \text { if } \quad \frac{\left|r-r_{n}\right|}{r_{n}} \geq \frac{1}{2} .
$$

The proof of (6.49) is thus complete.

It is now easy to conclude the proof of Lemma 4.6. The term $\mathcal{R}_{n}^{(3)}$ in the remainder is estimated using the lower bound (6.50), which leads to

$$
\left|\mathcal{R}_{n}^{(3)}(r)\right| \leq \frac{C}{r^{3}\left|r-r_{n}+i c_{n}\right|}, \quad r \geq \delta r_{n}
$$

whereas $\left|\mathcal{R}_{n}^{(1)}(r)\right| \leq C / r^{2}$ for all $r>0$. In view of (6.45), we thus have

$$
\left|\mathcal{K}_{n}^{(1)}(r, s)\right| \leq C \min \left(1,\left|s-r_{n}+i c_{n}\right|\right)^{1-2 \nu_{n}}\left(\frac{1}{s^{2}}+\frac{1}{s\left|s-r_{n}+i c_{n}\right|}\right), \quad s \geq r \geq \delta r_{n} .
$$

Integrating this bound, we arrive at

$$
\sup _{r \geq \delta r_{n}} \int_{r}^{\infty}\left|\mathcal{K}_{n}^{(1)}(r, s)\right| \mathrm{d} s \leq \frac{C}{r_{n}}\left(1+\log _{+}\left(r_{n}\right)\right) \leq C b_{n}^{1 / 2}\left(1+\log _{+} \frac{1}{b_{n}}\right),
$$

and estimate (4.32) follows immediately from (6.48), (6.52). 


\subsection{Proof of equality (4.42).}

Assume that $0<\nu<1 / 2$. Given any $\epsilon>0$ and $a>0$, we define

$$
\mathcal{J}_{\nu}(a)=\int_{-\epsilon}^{\epsilon} \frac{-a x}{\left(a^{2}+x^{2}\right)^{3 / 2}}\left|K_{\nu}(x+i a)\right|^{2} \mathrm{~d} x,
$$

where $K_{\nu}$ is the modified Bessel function. Our goal is to compute the limit of $\mathcal{J}_{\nu}(a)$ as $a \rightarrow 0$. We recall [1, Section 9.6] that

$$
K_{\nu}(z)=\frac{\pi}{2} \frac{I_{-\nu}(z)-I_{\nu}(z)}{\sin (\nu \pi)}, \quad \text { where } \quad I_{\nu}(z)=\frac{1}{\Gamma(1+\nu)} \frac{z^{\nu}}{2^{\nu}}\left(1+\mathcal{O}\left(z^{2}\right)\right) \quad \text { as } z \rightarrow 0 .
$$

We thus have

$$
K_{\nu}(z)=\frac{c_{\nu}}{z^{\nu}}\left(1-d_{\nu} z^{2 \nu}+\mathcal{O}\left(z^{2}\right)\right) \text { as } z \rightarrow 0
$$

where

$$
c_{\nu}=\frac{\pi}{\sin (\nu \pi)} \frac{2^{\nu-1}}{\Gamma(1-\nu)}, \quad d_{\nu}=\frac{1}{2^{2 \nu}} \frac{\Gamma(1-\nu)}{\Gamma(1+\nu)}
$$

It follows that

$$
\begin{aligned}
\left|K_{\nu}(x+i a)\right|^{2} & =\frac{c_{\nu}^{2}}{\left(a^{2}+x^{2}\right)^{\nu}}\left(\left|1-d_{\nu}(x+i a)^{2 \nu}\right|^{2}+\mathcal{O}\left(x^{2}+a^{2}\right)\right) \\
& =\frac{c_{\nu}^{2}}{\left(a^{2}+x^{2}\right)^{\nu}}\left(1-2 d_{\nu}\left(a^{2}+x^{2}\right)^{\nu} \cos (2 \nu \arg (x+i a))+\mathcal{O}\left(\left(x^{2}+a^{2}\right)^{2 \nu}\right)\right)
\end{aligned}
$$

as $z=x+i a \rightarrow 0$. The leading term in (6.54) is even and therefore does not contribute to the integral (6.53), where it is multiplied by an odd function. The main contribution comes from the next term, so that

$$
\begin{aligned}
\lim _{a \rightarrow 0} \mathcal{J}_{\nu}(a) & =2 d_{\nu} c_{\nu}^{2} \lim _{a \rightarrow 0} \int_{-\epsilon}^{\epsilon} \frac{a x}{\left(a^{2}+x^{2}\right)^{3 / 2}} \cos (2 \nu \arg (x+i a)) \mathrm{d} x \\
& =2 d_{\nu} c_{\nu}^{2} \int_{\mathbb{R}} \frac{y}{\left(1+y^{2}\right)^{3 / 2}} \cos (2 \nu \arg (y+i)) \mathrm{d} y
\end{aligned}
$$

where the second equality is obtained by setting $x=a y$.

It remains to perform the integral in (6.55). As $\arg (y+i)=\pi / 2-\arctan (y)$, we have

$$
\cos (2 \nu \arg (y+i))=\cos (\nu \pi) \cos (2 \nu \arctan (y))+\sin (\nu \pi) \sin (2 \nu \arctan (y)) .
$$

The first term does not contribute to the integral in (6.55), whereas setting $y=\tan (t)$ we find

$$
\int_{\mathbb{R}} \frac{y}{\left(1+y^{2}\right)^{3 / 2}} \sin (2 \nu \arctan (y)) \mathrm{d} y=2 \int_{0}^{\pi / 2} \sin (t) \sin (2 \nu t) \mathrm{d} t=\frac{4 \nu \cos (\nu \pi)}{1-4 \nu^{2}} .
$$

Summarizing, we have shown that

$$
\lim _{a \rightarrow 0} \mathcal{J}_{\nu}(a)=2 d_{\nu} c_{\nu}^{2} \sin (\nu \pi) \frac{4 \nu \cos (\nu \pi)}{1-4 \nu^{2}}=\frac{2 \pi \cos (\nu \pi)}{1-4 \nu^{2}} .
$$




\subsection{Explicit calculations in some particular cases}

We collect in this section a few results for the Kaufmann-Scully vortex (1.8) and the Lamb-Oseen vortex (1.9) which can be established by a direct calculation.

1. We first show that the vorticity profile $W$ of the Lamb-Oseen vortex satisfies assumption H2 in Section 1.2, hence belongs to the class $\mathscr{W}$. Indeed, in that case, the function $J$ defined by $(1.20)$ is given by

$$
J(r)=\frac{r^{4} e^{-r^{2}}\left(1-e^{-r^{2}}\right)}{\left(1-\left(1+r^{2}\right) e^{-r^{2}}\right)^{2}}, \quad r>0,
$$

so that

$$
J^{\prime}(r)=\frac{2 r^{3} e^{-r^{2}}}{\left(1-\left(1+r^{2}\right) e^{-r^{2}}\right)^{3}}\left(2-r^{2}-\left(4-r^{2}+r^{4}\right) e^{-r^{2}}+2 e^{-2 r^{2}}\right), \quad r>0 .
$$

We want to show that $J^{\prime}(r)<0$ for all $r>0$. Setting $s=r^{2}$, we have to verify that

$$
s-2+\left(4-s+s^{2}\right) e^{-s}-2 e^{-2 s}>0, \quad s>0,
$$

or equivalently

$$
\frac{s}{1-e^{-s}}+\left(\frac{s}{1-e^{-s}}\right)^{2} e^{-s}>2, \quad s>0 .
$$

Since $s>1-e^{-s}$, it is sufficient to show that

$$
\frac{s}{\tanh (s / 2)} \equiv \frac{s}{1-e^{-s}}+\frac{s e^{-s}}{1-e^{-s}}>2
$$

which is indeed true because $\tanh (x)<x$ for all $x>0$.

2. Next, for the Lamb-Oseen vortex, we establish the inequality $\mathcal{B}(r) \geq 1-4 / m^{2}$ when $a=0$ and $b \leq 0$, where $\mathcal{B}$ is defined in (3.3). Indeed, as $\gamma_{\star}(r)=\Omega(r)-b \geq \Omega(r)$, we have

$$
\mathcal{B}(r) \geq 1-\frac{1}{m^{2}} \frac{k^{2} r^{2}}{m^{2}+k^{2} r^{2}} \frac{\Phi}{\Omega^{2}}+\frac{r}{\Omega} \frac{W^{\prime}}{m^{2}+k^{2} r^{2}}-\frac{2 k^{2} r^{2}}{\Omega} \frac{W}{\left(m^{2}+k^{2} r^{2}\right)^{2}} .
$$

As $\Phi=2 \Omega W$ and $W^{\prime}(r)=-2 r W(r)$ for the Lamb-Oseen vortex, inequality (6.56) can be written in the equivalent form

$$
\mathcal{B}(r) \geq 1-\frac{2 W(r)}{m^{2} \Omega(r)}\left\{1+\frac{m^{2} r^{2}}{m^{2}+k^{2} r^{2}}-\frac{m^{4}}{\left(m^{2}+k^{2} r^{2}\right)^{2}}\right\} .
$$

Denote $s=r^{2}>0$ and $\alpha=m^{2} /\left(m^{2}+k^{2} r^{2}\right) \in(0,1)$. As $W=2 e^{-s}$ and $\Omega=s^{-1}\left(1-e^{-s}\right)$, it is straightforward to verify that (6.57) implies the desired inequality $\mathcal{B}(r) \geq 1-4 / m^{2}$ provided

$$
\frac{s e^{-s}}{1-e^{-s}}\left(1+\alpha s-\alpha^{2}\right) \leq 1, \quad \text { or equivalently } \quad e^{s} \geq 1+s+\alpha s(s-\alpha) .
$$

But

$$
\sup _{\alpha \in(0,1)}(1+s+\alpha s(s-\alpha))= \begin{cases}1+s+s^{3} / 4 & \text { if } s \leq 2, \\ 1+s^{2} & \text { if } s \geq 2\end{cases}
$$

and we conclude that the last inequality in (6.58) holds in all cases. 
3. Finally, we establish the same inequality $\mathcal{B}(r) \geq 1-4 / m^{2}$ for the Kaufmann-Scully vortex. In that case $W^{\prime}(r)=-4 r W(r) \Omega(r)$ by (1.8), hence inequality (6.56) takes the form

$$
\mathcal{B}(r) \geq 1-\frac{2 W(r)}{m^{2} \Omega(r)}\left\{1+\frac{2 r^{2}}{1+r^{2}} \frac{m^{2}}{m^{2}+k^{2} r^{2}}-\frac{m^{4}}{\left(m^{2}+k^{2} r^{2}\right)^{2}}\right\} .
$$

Setting again $\alpha=m^{2} /\left(m^{2}+k^{2} r^{2}\right)$ and using the fact that $W=2 \Omega^{2}=2 /\left(1+r^{2}\right)$ in the present case, we see that the desired inequality $\mathcal{B}(r) \geq 1-4 / m^{2}$ holds provided

$$
1+\frac{2 \alpha r^{2}}{1+r^{2}}-\alpha^{2} \leq 1+r^{2}, \quad r>0 .
$$

The maximum of the left-hand side, considered as a function of $\alpha \in(0,1)$, is reached at the point $\alpha=r^{2} /\left(1+r^{2}\right)$, and the resulting inequality becomes $\left(1+r^{2}\right)^{2}+r^{4} \leq\left(1+r^{2}\right)^{3}$, which is of course true. This concludes the proof.

\section{References}

[1] M. Abramowitz and I. Stegun, Handbook of Mathematical Functions, Dover, 1964.

[2] S. V. Alekseenko, P. A. Kuibin and V. L. Okulov, Theory of Concentrated Vortices. An Introduction, Springer, 2007.

[3] V. I. Arnold, Conditions for the nonlinear stability of the stationary plane curvilinear flows of an ideal fluid, Dokl. Mat. Nauk. 162 (1965), 773-777.

[4] V. I. Arnold, Sur la géométrie différentielle des groupes de Lie de dimension infinie et ses applications à l'hydrodynamique des fluides parfaits, Ann. Inst. Fourier 16 (1966), 319-361.

[5] P. Billant and F. Gallaire, Generalized Rayleigh criterion for non-axisymmetric centrifugal instabilities, J. Fluid Mech. 542 (2005), 365-379.

[6] E.A. Coddington and N. Levinson, Theory of ordinary differential equations, McGraw-Hill, New York, 1955.

[7] P. Drazin and W. Reid, Hydrodynamic stability, Cambridge Univ. Press, 1981.

[8] D. E. Edmunds and W. D. Evans, Spectral Theory and Differential Operators, second edition, Oxford university press, Oxford, 2018.

[9] D. Fabre, D. Sipp, and L. Jacquin, Kelvin waves and the singular modes of the Lamb-Oseen vortex, J. Fluid Mech. 551 (2006), 235-274.

[10] Th. Gallay and C. E. Wayne, Global Stability of vortex solutions of the two-dimensional Navier-Stokes equation, Comm. Math. Phys. 255 (2005), 97-129.

[11] Th. Gallay and D. Smets, On the linear stability of vortex columns in the energy space, arXiv:1811.07584.

[12] B. Helffer and J. Sjöstrand, Multiple wells in the semi-classical limit I, Commun. PDE 9 (1984), 337-408.

[13] L. N. Howard, Note on a paper of John W. Miles, J. Fluid Mech. 10 (1961), 509-512.

[14] L. N. Howard and A. S. Gupta, On the hydrodynamic and hydromagnetic stability of swirling flows, J. Fluid Mechanics 14 (1962), 463-476.

[15] T. Kato, Perturbation theory for linear operators, Grundlehren der mathematischen Wissenschaften 132, Springer, New York, 1966.

[16] S. Le Dizès and L. Lacaze, An asymptotic description of vortex Kelvin modes, J. Fluid Mech. 542 (2005), 69-96. 
[17] S. Leibovich and K. Stewartson K, A sufficient condition for the instability of columnar vortices, J. Fluid Mech 126 (1983), 335-356.

[18] J. W. Miles, On the stability of heterogeneous shear flows, J. Fluid Mech. 10 (1961), 496508.

[19] C. Marchioro and M. Pulvirenti, Mathematical theory of incompressible nonviscous fluids, Applied mathematical sciences 96, Springer, 1994.

[20] Lord Rayleigh, On the stability, or instability, of certain fluid motions, Proceedings of the London Mathematical Society 11 (1880), 57-72.

[21] Lord Rayleigh, On the dynamics of revolving fluids, Proceedings of the Royal Society A 93 (1917), 148-154.

[22] P. Rouchon, On the Arnold stability criterion for steady-state flows of an ideal fluid, Eur. J. Mech. B/Fluids 10 (1991), 651-661.

[23] B. Simon, Semiclassical analysis of low lying eigenvalues I. Non-degenerate minima: asymptotic expansions, Ann. Inst. Henri Poincaré A 38 (1983), 295-307.

[24] J. L. Synge, The stability of heterogeneous liquids, Trans. Roy. Soc. Canada 27 (1933), $1-18$.

[25] A. Szeri and P. Holmes, Nonlinear stability of axisymmetric swirling flows, Phil. Trans. R. Soc. London A 326 (1988), 327-354.

[26] G. I. Taylor, Effect of variation in density on the stability of superposed streams of fluid, Proc. Royal Soc. A 132 (1931), 499-523.

[27] Sir W. Thomson (Lord Kelvin), Vibrations of a columnar vortex, Proceedings of the Royal Society Edinburgh 10 (1880), 443-456. The London, Edinburgh and Dublin Philosophical Magazine and Journal of Science X (1880), 153-168. 